Neutron and Charged-Particle Induced Cross Sections for Radiochemistry in the Region of Samarium, Europium, and Gadolinium

R. D. Hoffman, K. Kelley, F. S. Dietrich, R. Bauer, M. Mustafa

April 20, 2005 
This document was prepared as an account of work sponsored by an agency of the United States Government. Neither the United States Government nor the University of California nor any of their employees, makes any warranty, express or implied, or assumes any legal liability or responsibility for the accuracy, completeness, or usefulness of any information, apparatus, product, or process disclosed, or represents that its use would not infringe privately owned rights. Reference herein to any specific commercial product, process, or service by trade name, trademark, manufacturer, or otherwise, does not necessarily constitute or imply its endorsement, recommendation, or favoring by the United States Government or the University of California. The views and opinions of authors expressed herein do not necessarily state or reflect those of the United States Government or the University of California, and shall not be used for advertising or product endorsement purposes.

This work was performed under the auspices of the U.S. Department of Energy by University of California, Lawrence Livermore National Laboratory under Contract W-7405-Eng-48. 
UCRL-TR-211588

\title{
Neutron and Charged-Particle Induced Cross Sections for Radiochemistry in the Region of Samarium, Europium and Gadolinium
}

\author{
R.D. Hoffman, K. Kelley, F. S. Dietrich and R. Bauer \\ Nuclear Theory and Modeling Group \\ Physics and Advanced Technologies, N-Division \\ Lawrence Livermore National Laboratory \\ Livermore, CA 94550 \\ rdhoffman@llnl.gov \\ M. Mustafa \\ Nuclear and Defense Technologies, AX-Division \\ Lawrence Livermore National Laboratory \\ Livermore, CA 94550
}

\begin{abstract}
We have developed a set of modeled nuclear reaction cross sections for use in radiochemical diagnostics. Systematics for the input parameters required by the Hauser-Feshbach statistical model were developed and used to calculate neutron and proton induced nuclear reaction cross sections in the mass region of samarium, europium and gadolinium $(62 \leq \mathrm{Z} \leq 64,82 \leq \mathrm{N} \leq 96)$.
\end{abstract}

Subject headings: Nuclear cross sections, Radiochemistry, Nuclear Physics

\section{Introduction}

\subsection{Radiochemistry}

Various aspects of nuclear explosive device performance can be determined through the use of radiochemistry. During the UGT (Under Ground Test) Program, select naturally occurring elements were often loaded into a device prior to a test and their activation products subsequently retrieved for counting. The products are measured as isotopic ratios (such as ${ }^{87} \mathrm{Y} /{ }^{88} \mathrm{Y}$ produced from a stable isotope of the naturally occurring element). From the measured activity and prior knowledge of the amount of loaded detector material, performance aspects could be inferred by comparing the measured isotope ratios with those calculated using neutron and charged-particle fluences from

\footnotetext{
${ }^{1}$ Department of Physics, University of California, Davis Davis, CA 95616
}

one of the design codes and group-averaged cross section sets that have been prepared for this purpose.

This is the third in a series of papers that details a collaborative effort between AX-Division and NDivision (PAT) to update and improve the existing RADCHEM charged particle cross section detector sets. The previous two concentrated on the noble gas detector sets Br-Kr (Hoffman et al. 2004a) and I-Xe (Hoffman et al. 2004b). Here we focus on the rare earth elements samarium, europium, and gadolinium. Only the unclassified cross section modelling effort is described. A separate classified document will discuss Stockpile Stewardship applications. 


\section{Contents}

1 Introduction $\quad 1$

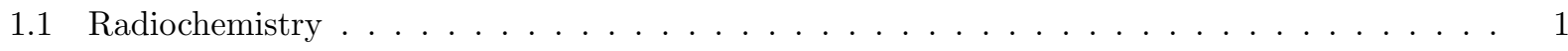

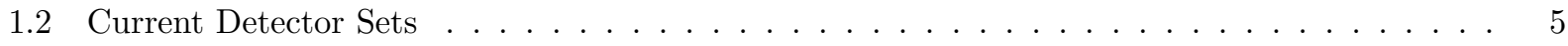

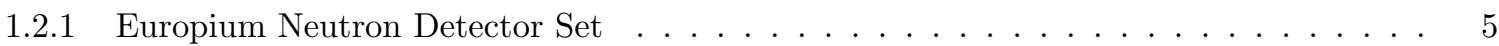

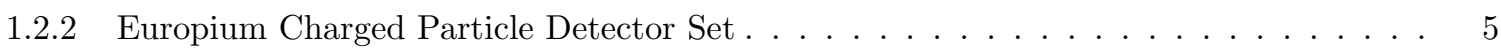

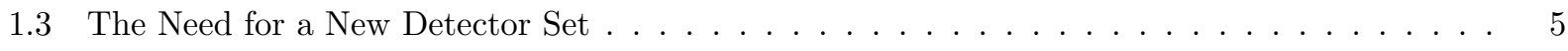

1.4 Proposed Rare-Earth Detector Sets . . . . . . . . . . . . . . . . . . . . 6

2 Nuclear Reaction Theory $\quad 7$

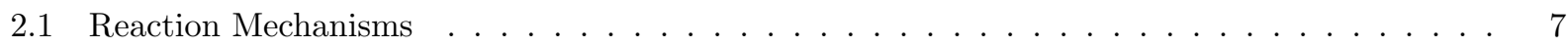

2.2 Hauser-Feshbach Statistical Model . . . . . . . . . . . . . . . . . . 7

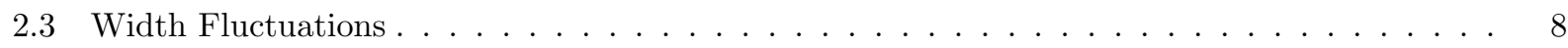

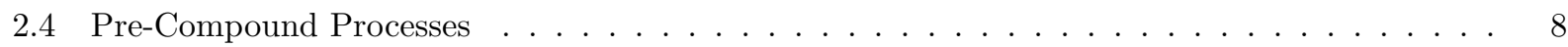

2.5 The STAPRE Hauser-Feshbach Reaction Code . . . . . . . . . . . . . . . . . 8

3 Inputs to the Hauser-Feshbach Model $r$

3.1 Nuclear Structure Data . . . . . . . . . . . . . . . . . . . . . . . . . . 8

3.1 .1 Nuclear Masses and $J^{\pi}$ Assignments . . . . . . . . . . . . . . . . . . . . 8

3.1 .2 Nuclear Level Schemes . . . . . . . . . . . . . . . . . . . . . . . . . . . . . . . . . . . .

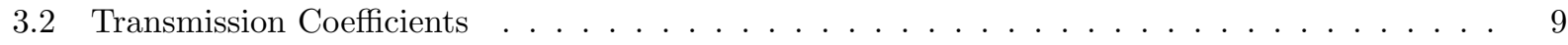

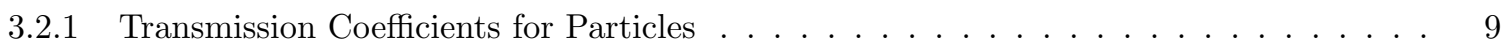

3.2.2 Considerations Regarding Collectivity and Nuclear Deformations . . . . . . . . . . . . 9

3.2 .3 The Neutron and Proton Optical Potential _ . . . . . . . . . . . . . . . . 9

3.2.4 Evaluation of the Neutron and Proton Optical Potential . . . . . . . . . . . . . . . 10

3.2.5 The Alpha and Deuteron Optical Potentials . . . . . . . . . . . . . . . . . . . 11

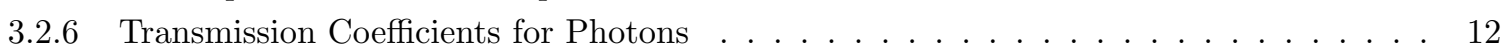

3.3 Nuclear Level Densities . . . . . . . . . . . . . . . . . . . . . . . . 13

3.3 .1 Level Density Models . . . . . . . . . . . . . . . . . . . . . . . 13

3.3.2 Level Densities Above the Neutron Binding Energy . . . . . . . . . . . . . . . . . . 14

The Spin Cutoff Parameter . . . . . . . . . . . . . . . . . . . 14

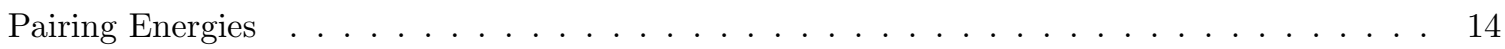

The Level Density Parameter . . . . . . . . . . . . . . . . . . . . . 14

Shell Corrections . . . . . . . . . . . . . . . . . . . . . . 14

3.3.3 Systematic Behavior of Fermi Gas Level Density Parameters . . . . . . . . . . . . 15

3.3.4 Level Densities Below the Neutron Binding Energy . . . . . . . . . . . . . . . . 15

Behavior of the Spin Cutoff Parameter Below $E_{x} \ldots \ldots \ldots \ldots \ldots \ldots$

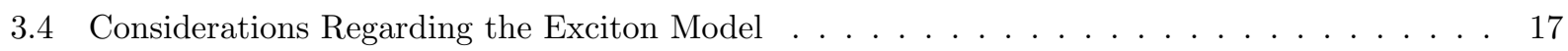

4 Calculated Cross Sections $r$

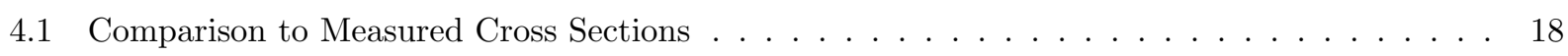

4.1.1 Comparison to experimental $(\mathrm{n}, \gamma)$ capture cross sections . . . . . . . . . . . . 18

4.1.2 Comparison to Maxwellian averaged $(\mathrm{n}, \gamma)$ capture cross sections $\ldots \ldots \ldots \ldots$. . . . . 20

4.1.3 Comparison to experimental $(\mathrm{n}, 2 \mathrm{n})$ cross sections . . . . . . . . . . . . . . 21

Uncertainty in the ${ }^{150} \mathrm{Eu}$ ground state vs isomer assignment . . . . . . . . . . . . . . . . . . . . . . . 22

4.1.4 Comparison to experimental $(\mathrm{p}, \mathrm{n})$ cross sections $\ldots \ldots \ldots \ldots \ldots \ldots$ 
4.1.5 Comparison to experimental $(\mathrm{d}, 2 \mathrm{n})$ cross sections . . . . . . . . . . . . . . . . . 24

4.1.6 Comparison to other experimental $(\mathrm{n}, \mathrm{x})$ cross sections . . . . . . . . . . . . . . 25

4.2 Sensitivity Studies . . . . . . . . . . . . . . . . . . . . . . . 25

4.2.1 Sensitivity to the Pre-Equilibrium Cross Section . . . . . . . . . . . . . . . . 25

4.2.2 Sensitivity to the Level Density - Various Prescriptions . . . . . . . . . . . . . . . 25

4.2.3 Sensitivity to the Level Density - Shell Correction Systematic . . . . . . . . . . . . . . 28

4.2.4 Sensitivity of $(\mathrm{d}, 2 \mathrm{n})$ Cross Sections to the Deuteron Optical Potential . . . . . . . . . 28

4.2.5 Sensitivity to the Normalization of the $\gamma$-ray Transmission Coefficient . . . . . . . . 30

4.2.6 Sensitivity to the Inclusion of Width Fluctuation Corrections . . . . . . . . . . . . . . 31

4.2.7 Net Uncertainties in $(\mathrm{n}, \gamma)$ Activation Cross Sections Due to Local Systematics . . . . 31

4.3 Production and Destruction Cross Sections . . . . . . . . . . . . . . . . . . 33

5 Conclusions $\quad 34$

5.0 .1 Recent LLNL experimental efforts . . . . . . . . . . . . . . . . . . . . . . 35

A Basic Nuclear Structure Data 38

A.1 New Samarium-Europium-Gadolinium Detector Set . . . . . . . . . . . . . . . . . 38

A.2 Binding and Separation Energies . . . . . . . . . . . . . . . . . . . . . . . . . 44

A.3 Q-values for Select Reactions . . . . . . . . . . . . . . . . . . . . . . . . 42

A.4 Adopted Level Schemes . . . . . . . . . . . . . . . . . . . . . . . . . . . . . . 43

A.5 Nuclear Level Density Parameters . . . . . . . . . . . . . . . . . . . . . . . 65

B Modeled Cross Sections vs. Experiment 68

C Modeled Cross Sections: Production and Destruction Channels 82

\section{List of Figures}



2 Total measured neutron cross sections vs. Optical Potential for ${ }^{144} \mathrm{Sm}+\mathrm{n}$ and ${ }^{148} \mathrm{Sm}+\mathrm{n}$. . . . 11

3 Comparison of predicted and experimental s- and p-wave strength functions. . . . . . . . . . 11

4 Comparison of predicted and experimental mean scattering radii. . . . . . . . . . . . . . . 11

5 Systematics for average total s-wave radiation width. . . . . . . . . . . . . . . . . . . 13

$6 \quad \chi^{2}$ linear fit to experimentally determined shell corrections, used to systematically determine unknown shell corrections. . . . . . . . . . . . . . . . . . . . . . . . . 15

7 Constant temperature level density fits to the low lying spectroscopic levels of ${ }^{151,153} \mathrm{Eu}$ and 151,153 Gd. . . . . . . . . . . . . . . . . . . . . . . . 16

8 Sensitivity of ${ }^{151,153} \mathrm{Eu}(\mathrm{n}, 2 \mathrm{n}),(\mathrm{n}, \gamma),(\mathrm{n}, \mathrm{p})$ and $(\mathrm{p}, \mathrm{n})$ cross sections to the alpha pre-formation

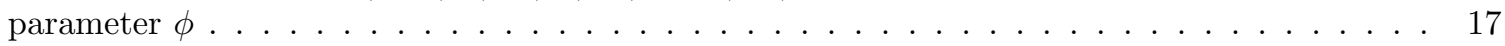

$9 \quad$ Calculated vs. experimental $(\mathrm{n}, \gamma),(\mathrm{n}, 2 \mathrm{n}),(\mathrm{p}, \mathrm{n})$ and $(\mathrm{d}, 2 \mathrm{n})$ cross sections: ${ }^{151,153}$ Eu. . . . . . 19

10 Systematics for a secondary normalization of the photon transmission coefficient based on replicating the 30-keV Maxwellian-averaged neutron capture cross sections . . . . . . . . . . . 21

11 Comparison of pre-equilibrium fractions resulting from the ALICE Monte Carlo model and the simple exciton model in STAPRE. . . . . . . . . . . . . . . . . . . . . . . . . 24

12 Sensitivity to pre-equilibrium matrix element FM. . . . . . . . . . . . . . . . . . . . 26

13 Sensitivity to the choice of level density prescription $\rho(U) \ldots \ldots$. . . . . . . . . . . . . 27



15 Sensitivity of $(\mathrm{d}, 2 \mathrm{n})$ cross sections to choice of deuteron optical potential (upper two panels) and the inclusion/exclusion of deuteron breakup (lower two panels). . . . . . . . . . . . 30 
16 Sensitivity to a $\pm 30 \%$ adjustment of the experimental s-wave average photon width $\Gamma_{\gamma} . \quad \ldots \quad 31$

17 Sensitivity to inclusion or exclusion of width fluctuation corrections $W \ldots \ldots \ldots$

18 Uncertainties in select $30 \mathrm{keV}(\mathrm{n}, \gamma)$ activation cross sections due to simultaneous variations

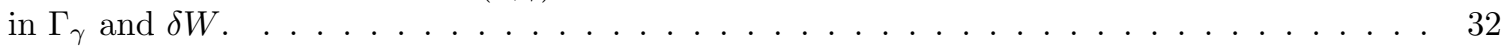

19 Calculated cross sections directly affecting production and destruction of ${ }^{151,153} \mathrm{Gd}$. . . . . . 34

20 Adopted level schemes for $\mathrm{Sm}, \mathrm{Eu}$, and $\mathrm{Gd}$ nuclei. . . . . . . . . . . . . . . . . . 44

21 Measured vs. calculated $(\mathrm{n}, \gamma)$ cross sections on select targets. . . . . . . . . . . . . 69

22 Measured vs. calculated Maxwellian averaged $(\mathrm{n}, \gamma)$ cross sections. $\ldots \ldots \ldots \ldots \ldots$

23 Measured vs. calculated $(\mathrm{n}, 2 \mathrm{n})$ cross sections for select targets. . . . . . . . . . . . . 75

24 Measured vs. calculated $(\mathrm{n}, 3 \mathrm{n})$ cross sections for select targets. . . . . . . . . . . . . 77

25 Measured vs. calculated $(\mathrm{n}, \mathrm{p})$ cross sections for select targets. . . . . . . . . . . . . 78

26 Measured vs. calculated $(\mathrm{n}, \alpha)$ and $(\mathrm{n}, \mathrm{d})$ cross sections for select targets. . . . . . . . . . 80

27 Production and destruction cross sections for $\mathrm{Sm}, \mathrm{Eu}, \& \mathrm{Gd}$ targets $\ldots \ldots \ldots$. . . . . . . 83

\section{List of Tables}

1 Cross sections available in RADCHEM detector set Eu0289 . . . . . . . . . . . . . 5





4 Experimental vs. calculated total neutron cross sections at $14.2 \mathrm{MeV} \ldots \ldots \ldots \ldots$

5 Comparison of our modeled $(\mathrm{n}, \gamma)$ cross sections to experimental data at $30 \pm 2 \mathrm{keV} \ldots \ldots . \quad 20$

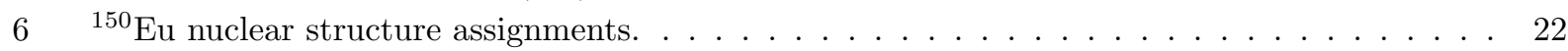

$7 \quad$ Comparison of our modeled $(\mathrm{n}, 2 \mathrm{n})$ cross sections to experimental data at $14.7 \pm 0.1 \mathrm{MeV}$. . . 23

8 Uncertainties in select $(\mathrm{n}, \gamma) 30 \mathrm{keV}$ activation cross sections arising from local systematics, expressed as an absolute percent difference from our recommended values . . . . . . . . . . 33

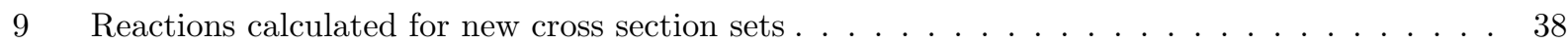

10 Spins, parities, binding energies, and separation energies for new samarium and europium sets 40

11 Q-values $(\mathrm{MeV})$ for select reactions in the new samarium and europium sets . . . . . . . . 42

12 Level density parameters . . . . . . . . . . . . . . . . . . . . . . . . 65 


\subsection{Current Detector Sets}

\subsubsection{Europium Neutron Detector Set}

Over the last 40 years a number of detector sets have been developed at LLNL and LANL. Twenty-three neutron threshold detector sets and five charged particle sets are currently available. The Europium set (Eu0289) is a neutron detector set used to calculate activation of ${ }^{147} \mathrm{Eu}\left(\tau_{1 / 2}=\right.$ $24.5 \mathrm{~d}),{ }^{148} \mathrm{Eu}\left(\tau_{1 / 2}=54.5 \mathrm{~d}\right),{ }^{149} \mathrm{Eu}\left(\tau_{1 / 2}=93.1\right.$ d), ${ }^{150 g} \mathrm{Eu}\left(\tau_{1 / 2}=12.8 \mathrm{~h}\right),{ }^{150 m} \mathrm{Eu}\left(\tau_{1 / 2}=35.8\right.$ y), ${ }^{152} \mathrm{Eu}\left(\tau_{1 / 2}=13.5 \mathrm{y}\right)$ and ${ }^{154} \mathrm{Eu}\left(\tau_{1 / 2}=8.59\right.$ y) from stable ${ }^{151} \mathrm{Eu}$ and ${ }^{153} \mathrm{Eu}$. It consists of 45 neutron reactions, largely taken from calculations done in the 1960's, with four $(\mathrm{n}, 2 \mathrm{n})$ reactions scaled to match measured values at 14.1 $\mathrm{MeV}$. The set is summarized in Table (1.2.1) and can be accessed on the world wide web, see http://nuclear.llnl.gov/CNP/nads/main.html.

\subsubsection{Europium Charged Particle Detector Set}

The Europium set (Eu0988) is a neutron and charged particle set used to calculate activation of ${ }^{151} \mathrm{Gd}\left(\tau_{1 / 2}=125.6 \mathrm{~d}\right)$ and ${ }^{153} \mathrm{Gd}$ $\left(\tau_{1 / 2}=237.9 \mathrm{~d}\right)$ from stable ${ }^{151} \mathrm{Eu}$ and ${ }^{153} \mathrm{Eu}$, and consists of 20 neutron and four charged particle reactions. The charged particle reactions are based on experimental data (West et al. 1993), (Nethaway \& Mustafa 1999), while the neutron reactions are based on a mixture of data and calculation. The set is summarized in Table (1.2.2), and also can be accessed on the world wide web, see http://nuclear.llnl.gov/CNP/nads/main.html.

\subsection{The Need for a New Detector Set}

The motivations for revisiting the detector sets for radiochemistry are many. The current sets were often developed based on "best guess" and "experience" from a limited amount of experimental data over a 40 year period. Often a single measurement at $14 \mathrm{MeV}$ guided the evaluation of a critical $(\mathrm{n}, 2 \mathrm{n})$ cross section, with an assumed shape that would rise from a calculated threshold, and then adjusted to match the experimental point at $14 \mathrm{MeV}$.

Of the 69 cross sections in the two europium sets, only ten are based on experimental measurements. Of the remaining cross sections, only 22 are modeled or calculated. The remaining 37 cross sections were copied from the experimental or modeled cross sections, with adjustments made to match single experimental data points and to rise from a calculated threshold.
Table 1: Cross sections available in RADCHEM detector set Eu0289.

\begin{tabular}{|c|c|c|}
\hline Set $\rightarrow$ & \multicolumn{2}{|c|}{ 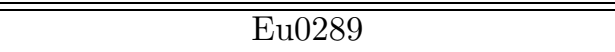 } \\
\hline \multirow{9}{*}{ Calv. } & ${ }^{146} \mathrm{Eu}(\mathrm{n}, 2 \mathrm{n})^{145} \mathrm{Eu}$ & ${ }^{148} \mathrm{Eu}(\mathrm{n}, \gamma){ }^{149} \mathrm{Eu}$ \\
\hline & ${ }^{147} \mathrm{Eu}(\mathrm{n}, 2 \mathrm{n}){ }^{146} \mathrm{Eu}$ & ${ }^{149 g} \mathrm{Eu}(\mathrm{n}, \gamma){ }^{150 g} \mathrm{Eu}$ \\
\hline & ${ }^{148} \mathrm{Eu}(\mathrm{n}, 2 \mathrm{n}){ }^{147} \mathrm{Eu}$ & ${ }^{150 g} \mathrm{Eu}(\mathrm{n}, \gamma){ }^{151 g} \mathrm{Eu}$ \\
\hline & ${ }^{149 g} \mathrm{Eu}(\mathrm{n}, 2 \mathrm{n})^{148} \mathrm{Eu}$ & ${ }^{151 g} \mathrm{Eu}(\mathrm{n}, \gamma)^{152 g} \mathrm{Eu}$ \\
\hline & ${ }^{150 g} \mathrm{Eu}(\mathrm{n}, 2 \mathrm{n})^{149 g} \mathrm{Eu}$ & ${ }^{152 g} \mathrm{Eu}(\mathrm{n}, \gamma){ }^{153} \mathrm{Eu}$ \\
\hline & ${ }^{151 g} \mathrm{Eu}(\mathrm{n}, 2 \mathrm{n})^{150 g} \mathrm{Eu}$ & ${ }^{153} \mathrm{Eu}(\mathrm{n}, \gamma){ }^{154} \mathrm{Eu}$ \\
\hline & ${ }^{152 g} \mathrm{Eu}(\mathrm{n}, 2 \mathrm{n})^{151 g} \mathrm{Eu}$ & ${ }^{154} \mathrm{Eu}(\mathrm{n}, \gamma){ }^{155} \mathrm{Eu}$ \\
\hline & ${ }^{153} \mathrm{Eu}(\mathrm{n}, 2 \mathrm{n}){ }^{152 g} \mathrm{Eu}$ & ${ }^{155} \mathrm{Eu}(\mathrm{n}, \gamma){ }^{156} \mathrm{Eu}$ \\
\hline & ${ }^{154} \mathrm{Eu}(\mathrm{n}, 2 \mathrm{n}){ }^{153} \mathrm{Eu}$ & \\
\hline \multirow{15}{*}{ Copied* } & ${ }^{149 m} \mathrm{Eu}(\mathrm{n}, 2 \mathrm{n})^{148} \mathrm{Eu}$ & ${ }^{148} \mathrm{Eu}(\mathrm{n}, \gamma){ }^{149 g} \mathrm{Eu}$ \\
\hline & ${ }^{150 g} \mathrm{Eu}(\mathrm{n}, 2 \mathrm{n})^{149 m} \mathrm{Eu}$ & ${ }^{149 g} \mathrm{Eu}(\mathrm{n}, \gamma){ }^{150 m} \mathrm{Eu}$ \\
\hline & ${ }^{150 m} \mathrm{Eu}(\mathrm{n}, 2 \mathrm{n})^{149 g} \mathrm{Eu}$ & ${ }^{149 m} \mathrm{Eu}(\mathrm{n}, \gamma){ }^{150 g} \mathrm{Eu}$ \\
\hline & ${ }^{150 m} \mathrm{Eu}(\mathrm{n}, 2 \mathrm{n})^{149 m} \mathrm{Eu}$ & ${ }^{149 m} \mathrm{Eu}(\mathrm{n}, \gamma)^{150 m} \mathrm{Eu}$ \\
\hline & ${ }^{151 g} \mathrm{Eu}(\mathrm{n}, 2 \mathrm{n}){ }^{150 m} \mathrm{Eu}$ & ${ }^{150 g} \mathrm{Eu}(\mathrm{n}, \gamma)^{151 m} \mathrm{Eu}$ \\
\hline & ${ }^{151 m} \mathrm{Eu}(\mathrm{n}, 2 \mathrm{n})^{150 g} \mathrm{Eu}$ & ${ }^{150 m} \mathrm{Eu}(\mathrm{n}, \gamma){ }^{151 g} \mathrm{Eu}$ \\
\hline & ${ }^{151 m} \mathrm{Eu}(\mathrm{n}, 2 \mathrm{n})^{150 m} \mathrm{Eu}$ & ${ }^{150 m} \mathrm{Eu}(\mathrm{n}, \gamma){ }^{151 m} \mathrm{Eu}$ \\
\hline & ${ }^{152} \mathrm{Eu}(\mathrm{n}, 2 \mathrm{n}){ }^{151 m} \mathrm{Eu}$ & ${ }^{151 g} \mathrm{Eu}(\mathrm{n}, \gamma){ }^{152 m 1} \mathrm{Eu}$ \\
\hline & ${ }^{152 m 1} \mathrm{Eu}(\mathrm{n}, 2 \mathrm{n}){ }^{151 g} \mathrm{Eu}$ & ${ }^{151 g} \mathrm{Eu}(\mathrm{n}, \gamma)^{152 m 2} \mathrm{Eu}$ \\
\hline & ${ }^{152 m 1} \mathrm{Eu}(\mathrm{n}, 2 \mathrm{n})^{151 m} \mathrm{Eu}$ & ${ }^{151 m} \mathrm{Eu}(\mathrm{n}, \gamma)^{152 g} \mathrm{Eu}$ \\
\hline & ${ }^{152 m 2} \mathrm{Eu}(\mathrm{n}, 2 \mathrm{n}){ }^{151 g} \mathrm{Eu}$ & ${ }^{151 m} \mathrm{Eu}(\mathrm{n}, \gamma)^{152 m 1} \mathrm{Eu}$ \\
\hline & ${ }^{152 m 2} \mathrm{Eu}(\mathrm{n}, 2 \mathrm{n})^{151 m} \mathrm{Eu}$ & ${ }^{151 m} \mathrm{Eu}(\mathrm{n}, \gamma){ }^{152 m 2} \mathrm{Eu}$ \\
\hline & ${ }^{153} \mathrm{Eu}(\mathrm{n}, 2 \mathrm{n}){ }^{152 m 1} \mathrm{Eu}$ & ${ }^{152 m 1} \mathrm{Eu}(\mathrm{n}, \gamma){ }^{153} \mathrm{Eu}$ \\
\hline & ${ }^{153} \mathrm{Eu}(\mathrm{n}, 2 \mathrm{n})^{152 m 2} \mathrm{Eu}$ & ${ }^{152 m 2} \mathrm{Eu}(\mathrm{n}, \gamma)^{153} \mathrm{Eu}$ \\
\hline & ${ }^{151 g} \mathrm{Eu}(\mathrm{n}, 2 \mathrm{n}){ }^{150 m} \mathrm{Eu}$ & ${ }^{151 m} \mathrm{Eu}(\mathrm{n}, 2 \mathrm{n})^{150 m} \mathrm{Eu}$ \\
\hline Scaled & ${ }^{151 g} \mathrm{Eu}(\mathrm{n}, 2 \mathrm{n})^{150 g} \mathrm{Eu}$ & ${ }^{151 m} \mathrm{Eu}(\mathrm{n}, 2 \mathrm{n})^{150 g} \mathrm{Eu}$ \\
\hline
\end{tabular}

* These cross sections are duplicates of other measured or calculated cross sections, e.g. the ${ }^{150 g} \mathrm{Eu}(\mathrm{n}, 2 \mathrm{n})^{149 m} \mathrm{Eu}$ cross section is a copy of the ${ }^{150 g} \mathrm{Eu}(\mathrm{n}, 2 \mathrm{n})^{149 g} \mathrm{Eu}$ cross section.

The majority of the modeled cross sections come from calculations made in the 1960's. Many of the neutron capture cross sections were estimated and/or extrapolated from data spanning energies up to $700 \mathrm{keV}$. Many of the $(\mathrm{n}, 2 \mathrm{n})$ calculations were scaled up or down to match limited data around $14 \mathrm{MeV}$ when such data was available. To correct for photon intensities in use from Test Program measurements, the charged particle production cross sections were also scaled up or down. Additionally, the charged particle cross sections are still based on preliminary data (Nethaway 1998).

In the neutron set (Eu0289), the 28 cross sections involving isomers were copied from the calculated ground state to ground state cross section (i.e. $\quad{ }^{150 g} \mathrm{Eu}(\mathrm{n}, 2 \mathrm{n})^{149 m} \mathrm{Eu}, \quad{ }^{150 m} \mathrm{Eu}(\mathrm{n}, 2 \mathrm{n})^{149 g} \mathrm{Eu}$ and ${ }^{150 m} \mathrm{Eu}(\mathrm{n}, 2 \mathrm{n})^{149 m} \mathrm{Eu}$ are all copied from 
Table 2: Cross sections available in RADCHEM detector set Eu0988.

\begin{tabular}{|c|c|}
\hline Set $\rightarrow$ & Eu0988 \\
\hline \multirow{10}{*}{ Measured } & ${ }^{151} \mathrm{Eu}(\mathrm{p}, \mathrm{n}){ }^{151} \mathrm{Gd}$ \\
\hline & ${ }^{153} \mathrm{Eu}(\mathrm{p}, \mathrm{n})^{153} \mathrm{Gd}$ \\
\hline & ${ }^{151} \mathrm{Eu}(\mathrm{d}, 2 \mathrm{n}){ }^{151} \mathrm{Gd}$ \\
\hline & ${ }^{153} \mathrm{Eu}(\mathrm{d}, 2 \mathrm{n})^{153} \mathrm{Gd}$ \\
\hline & ${ }^{151} \mathrm{Eu}(\mathrm{n}, \gamma){ }^{152} \mathrm{Eu}$ \\
\hline & ${ }^{153} \mathrm{Eu}(\mathrm{n}, \gamma){ }^{154} \mathrm{Eu}$ \\
\hline & ${ }^{151} \mathrm{Eu}(\mathrm{n}, 2 \mathrm{n}){ }^{150} \mathrm{Eu}$ \\
\hline & ${ }^{153} \mathrm{Eu}(\mathrm{n}, 2 \mathrm{n}){ }^{152} \mathrm{Eu}$ \\
\hline & ${ }^{152} \mathrm{Gd}(\mathrm{n}, \gamma){ }^{153} \mathrm{Gd}$ \\
\hline & ${ }^{154} \mathrm{Gd}(\mathrm{n}, \gamma){ }^{155} \mathrm{Gd}$ \\
\hline \multirow{5}{*}{ Calculated } & ${ }^{152} \mathrm{Eu}(\mathrm{n}, \gamma){ }^{153} \mathrm{Eu}$ \\
\hline & ${ }^{154} \mathrm{Eu}(\mathrm{n}, \gamma){ }^{155} \mathrm{Eu}$ \\
\hline & ${ }^{151} \mathrm{Gd}(\mathrm{n}, 2 \mathrm{n}){ }^{150} \mathrm{Gd}$ \\
\hline & ${ }^{152} \mathrm{Gd}(\mathrm{n}, 2 \mathrm{n}){ }^{151} \mathrm{Gd}$ \\
\hline & ${ }^{153} \mathrm{Gd}(\mathrm{n}, 2 \mathrm{n}){ }^{152} \mathrm{Gd}$ \\
\hline \multirow{9}{*}{ Copied* } & ${ }^{150} \mathrm{Eu}(\mathrm{n}, \gamma){ }^{151} \mathrm{Eu}$ \\
\hline & ${ }^{150} \mathrm{Eu}(\mathrm{n}, 2 \mathrm{n}){ }^{149} \mathrm{Eu}$ \\
\hline & ${ }^{152} \mathrm{Eu}(\mathrm{n}, 2 \mathrm{n})^{151} \mathrm{Eu}$ \\
\hline & ${ }^{154} \mathrm{Eu}(\mathrm{n}, 2 \mathrm{n}){ }^{153} \mathrm{Eu}$ \\
\hline & ${ }^{150} \mathrm{Gd}(\mathrm{n}, \gamma){ }^{151} \mathrm{Gd}$ \\
\hline & ${ }^{151} \mathrm{Gd}(\mathrm{n}, \gamma){ }^{152} \mathrm{Gd}$ \\
\hline & ${ }^{153} \mathrm{Gd}(\mathrm{n}, \gamma){ }^{154} \mathrm{Gd}$ \\
\hline & ${ }^{150} \mathrm{Gd}(\mathrm{n}, 2 \mathrm{n}){ }^{149} \mathrm{Gd}$ \\
\hline & ${ }^{154} \mathrm{Gd}(\mathrm{n}, 2 \mathrm{n}){ }^{153} \mathrm{Gd}$ \\
\hline
\end{tabular}

* These cross sections are duplicates of other measured or calculated cross sections, e.g. the ${ }^{150} \mathrm{Gd}(\mathrm{n}, \gamma)^{151} \mathrm{Gd}$ cross section is a copy of the ${ }^{152} \mathrm{Gd}(\mathrm{n}, \gamma){ }^{153} \mathrm{Gd}$ cross section.

$\left.{ }^{150 g} \mathrm{Eu}(\mathrm{n}, 2 \mathrm{n})^{149 g} \mathrm{Eu}\right)$. Some were then scaled up or down to match experimental data at $14 \mathrm{MeV}$. In the charged particle set, four capture cross sections and five $(\mathrm{n}, 2 \mathrm{n})$ cross sections were simply copied from the respective cross sections of nearby isotopes, with appropriate adjustments made to the threshold and scaled when data was available.

In the decade since this cross section set was last evaluated, many new cross section measurements have been performed, and several efforts have been made to develop consistent approaches to modeling nuclear reaction cross sections (RIPL 1998). The basic nuclear structure data has been greatly improved. Finally, there are more accurate methods of calculating and estimating cross sections for which we have no data.

\subsection{Proposed Rare-Earth Detector Sets}

We consider as targets all isotopes of the elements $\mathrm{Sm}, \mathrm{Eu}$, and $\mathrm{Gd}(62 \leq \mathrm{Z} \leq 64)$ with neutron numbers $82 \leq \mathrm{N} \leq 96$ (including any long-lived isomers with half-lives greater than 1 $\mu \mathrm{s}$ ), and have calculated nuclear reaction cross sections for incident neutrons, protons, and deuterons on these targets with laboratory incident particle energies ranging from $0.01 \mathrm{keV}$ to $20 \mathrm{MeV}$. These compound systems are then allowed to decay through the reaction channels shown in Table 9.

The reason for including many more isotopes than were included in the original sets is to account for the various possible destruction reactions that are significant in this mass range. In general, the current RADCHEM detector sets, and especially the charged-particle sets, were developed with special attention paid to the production reactions. We have also included the isomers as targets, which were not included in some of the original sets, in order to gauge the sensitivity of the set to their inclusion.

Another important reason for considering a larger range of nuclei is to compare our calculated cross sections to the many measured cross sections available for the stable samarium, europium, and gadolinium isotopes. Our goal is to develop a consistent set that reproduces, as closely as possible, measured cross sections on targets in the local region of interest. To do this we develop local systematics for the many input quantities used in the theoretical reaction modeling calculations. These systematics are based on experimental data that are often only available for compound nuclear systems formed from a stable target plus a neutron. Of course, we use experimental data whenever it is available, but reactions proceeding through unstable systems are unavoidable in radiochemistry. Short of developing new experimental techniques to measure cross sections on unstable targets, our only hope of reproducing measured activity from UGT shots, and addressing the uncertainty associated with the nuclear cross sections, is to develop cross section sets that reproduce well the measured cross sections in the local region of interest.

In $\S 2$ we describe the theoretical techniques used in the modeling effort. $\S 3$ describes the input parameters. $\S 4$ gives results. We conclude with $\S 5$. 


\section{Nuclear Reaction Theory}

\subsection{Reaction Mechanisms}

Conceptually, we consider nuclear reaction mechanisms to be of two general types, direct processes and compound processes. Direct processes can be pictured as simple interactions of the incident particle with the nuclear potential of the target nucleus. They proceed on a rapid time scale (of order $\sim 10^{-22} \mathrm{~s}$ ), and the reaction products are often highly peaked in the incident particle direction. Compound processes are pictured as complicated interactions proceeding over a much longer timescale $\left(10^{-15}-10^{-18} \mathrm{~s}\right)$ in which the reaction is mediated by the formation of a "compound nucleus", with the excitation energy of the incident particle being statistically "shared" with the ensemble of nucleons in the target over all energetically allowed degrees of freedom. The reaction products are largely isotropic. Compound nuclear reactions proceed through resonances, which correspond to nuclear states above the bound region, while direct reactions proceed through smooth potential terms. Other intermediate reaction mechanisms may exist between these two extremes. We refer to these as "precompound" nuclear processes. Over the energy range of interest to this project, a few $\mathrm{keV}$ to 20 $\mathrm{MeV}$, we will consider pre-compound and compound nuclear processes, with the pre-compound processes operating principally above $10 \mathrm{MeV}$ of incident particle excitation energy.

\subsection{Hauser-Feshbach Statistical Model}

A traditional theoretical approach to compound nuclear reactions is the statistical or HauserFeshbach model. This model is valid only for high level densities in the compound nucleus, allowing one to use energy averaged transmission coefficients $T$, which describe absorption via an imaginary part in the (optical) nucleon-nucleus potential (for details see Mahaux and Weidenmüller 1979). For the reaction I (in state $\mu$ ) $+j \rightarrow k+\mathrm{L}$ (in state $\nu$ ), with $\mathrm{I}^{\nu}+j$ interacting with centerof-mass energy $\mathrm{E}_{j}^{\mu}$ (in $\mathrm{MeV}$ ), the average cross section is given by

$$
\sigma_{j k}^{\mu \nu}\left(E_{j}^{\mu}\right)=\frac{\pi \lambda_{j}^{2}}{g_{I}^{\mu} g_{j}} \sum_{J, \pi} g_{J} \frac{T_{j}^{\mu}\left(J^{\pi}\right) T_{k}^{\nu}\left(J^{\pi}\right)}{T_{t o t}\left(J^{\pi}\right)} W\left(J^{\pi}\right)
$$

where the summation extends over all compound nuclear spins and parities $J^{\pi}, \mu$ and $\nu$ are states in the target and product ( $=0$ for the ground state, 1 for the $1^{\text {st }}$ excited state, etc.). The cross section has units of area, described by $\pi \lambda_{j}^{2}=$ $0.6566\left(\hat{A}_{j} E_{j}^{\mu}\right)^{-1}$ barns, with $\hat{A}_{j}=\left(A_{I} A_{j}\right) /\left(A_{I}+\right.$ $A_{j}$ ) being the reduced mass in atomic mass units and $E_{j}^{\mu}$ is the center of mass energy in units of $\mathrm{MeV} . \lambda_{j}$ is the wavelength related to the wave number $k_{j}$ in the target plus incident particle channel by $\lambda_{j}=1 / k_{j}$ The statistical weights are given by $g_{y}^{x}=\left(2 J_{y}^{x}+1\right)$. Items without superscripts refer to the compound nucleus.

The transmission coefficients in the numerator are given by $T_{j}^{\mu}\left(J^{\pi}\right)=$ the total transmission coefficient for forming the state $J^{\pi}$ in the compound nucleus $I^{\mu}+j$ at energy $E_{j}^{\mu}$. Likewise, $T_{k}^{\nu}\left(J^{\pi}\right)$ is the same as $T_{j}^{\mu}\left(J^{\pi}\right)$ but for the pair $L^{\nu}+k$ at energy $E_{k}^{\nu}$. Implicit in these definitions is a sum over all possible $l$-waves and channel spins, i.e.

$$
T_{j}^{\mu}\left(J^{\pi}\right)=\sum_{l, s} T_{j}^{\mu}\left(J^{\pi}, l, s\right)
$$

where $l$ is any partial wave number (orbital angular momentum) that can couple the state $\mu$ to the compound nuclear state having spin and parity $J^{\pi}$ subject to quantum mechanical selection rules and $s$ is the vector sum of the spins $J_{I}^{\mu}$ and $J_{j}$. Hence $s$ takes on all integer (or half-integer) numbers from $\left|J_{I}^{\mu}-J_{j}\right|$ to $J_{I}^{\mu}+J_{j}$.

$T_{\text {tot }}$ represents the sum of transmission coefficients over all possible decay channels (i.e. for all particles and photons). The cross section for the formation of species L, regardless of its state $\nu$, is obtained by summing Eq. [1] over all bound states $\nu$ of $\mathrm{L}$ for which the reaction is energetically allowed.

When evaluating these sums, if energies become of interest which exceed the highest discrete excited state for which energy, spin, and parity are explicitly known, a nuclear level density formula must be employed. Specifically, the definitions for the transmission coefficients $T_{j}\left(J^{\pi}\right), T_{k}\left(J^{\pi}\right)$, and $T_{t o t}\left(J^{\pi}\right)$ must be modified, for example:

$$
\begin{aligned}
& T_{k}\left(J^{\pi}\right)=\sum_{\nu=0}^{\omega} T_{k}^{\nu}\left(J^{\pi}\right)+ \\
& \sum_{J^{\nu} \pi^{\nu}} \int_{\xi_{L}^{\omega}}^{\xi_{L}^{\text {max }}} T_{k}^{\nu}\left(\xi_{L}^{\nu}, J^{\pi}\right) \rho\left(\xi_{L}^{\nu}, J^{\nu}, \pi^{\nu}\right) d \xi_{L}^{\nu} d \pi^{\nu} d J^{\nu}
\end{aligned}
$$

where for the nucleus $\mathrm{L}, \xi_{L}^{\omega}$ is the energy of the highest excited state, $\omega$, of known energy, spin, and parity; $\xi_{L}^{\max }=E_{k}^{0}=E_{j}^{0}+Q_{j k}$ is the maximum excitation energy available, and $\rho\left(\xi_{L}^{\nu}, J^{\nu}, \pi^{\nu}\right)$ 
is the density of states per unit energy of spin and parity $J^{\nu}$ and $\pi^{\nu}$ at the excitation energy $\xi_{L}^{\nu}$. The above integral approximates a summation and is subject to the same quantum mechanical restrictions implied in the definition of the transmission function.

\subsection{Width Fluctuations}

In addition to the ingredients required for Eq. [1], like the transmission coefficients for particles and photons or the level densities, width fluctuation corrections $\left(W\left(J^{\pi}\right)\right.$, hereafter WFC) have to be employed as well. They define the correlation factors with which all partial channels of incoming particle $j$ and outgoing particle $k$, passing through excited state $(E, J, \pi)$, have to be multiplied. The major effect is to enhance the elastic channel and accordingly decrease the other open channels. They are most often observed at or near channel opening energies when i.e. a $(p, \gamma)$ and a $(\mathrm{p}, \mathrm{n})$ channel compete and the weaker $(\mathrm{p}, \gamma)$ channel is enhanced. Above a few $\mathrm{MeV}$ of excitation energy, when many competing channels are open, WFC's can be neglected.

A reasonably complete treatment for the WFC, obtained with the Gaussian orthogonal ensemble (GOE) approach, requires the evaluation of a triple integral and to date has been considered much to costly to apply in nuclear cross section calculations. Several approximations have been developed, the most popular ones are the Moldauer model (Moldauer 1976), and the HRTW model (Hofmann et al. 1975). We use the Moldauer model approximation in this study. For a detailed description of the full (GOE) treatment and a comparison with the Moldauer and HRTW approximation models mentioned above, see (Hilaire Lagrange \& Koning 2003).

\subsection{Pre-Compound Processes}

For excitation energies starting around $10 \mathrm{MeV}$, pre-compound processes become important. The pre-compound cross section is subtracted from the total cross section of the first compound nucleus, and is usually unimportant for subsequent compound nuclei. Here we describe equilibration of the compound nuclear system in terms of a simple exciton model. In the pre-equilibrium stage of the reaction particle emission is assumed to be the only decay mode. For the equilibration portion of the first chance particle or photon emission as well as for first chance fission, the width fluctuated Hauser Feshbach formula (Eq. [1]) is applied. All subsequent (higher chance) processes are treated as sequential evaporation steps.

\subsection{The STAPRE Hauser-Feshbach Reac- tion Code}

We adopt the statistical model code STAPRE (STAatistical-PREequilibrium) to model our cross sections (Uhl \& Strohmaier 1976). It embodies all of the physical models discussed above. The version of the code we use is STAPRE-H95 (Avrigeanu \& Avrigeanu 1976), available from the NEA web site. We have made several modifications, primarily to the level density routines. Prior versions of the code were used to develop parts of the existing RADCHEM data sets (Vonach 1982).

In the following we discuss the important ingredients of statistical model calculations, and the methods utilized to estimate them. These are the requisite nuclear structure data, such as the binding energies of all nuclei included (which define the separation and reaction threshold energies and Q-values of the various reaction channels considered), as well as the energies, spins, and parities of the ground states and all known excited states of these nuclei, and the detailed branching ratios for the gamma-ray cascade from excited to lowlying states. Also needed are prescriptions for the width fluctuation corrections, the pre-compound cross section, the particle and $\gamma$-transmission coefficients, and the nuclear level densities of all nuclei involved in a given reaction. The reliability with which these ingredients can be calculated determines the accuracy of a given cross section calculation.

\section{Inputs to the Hauser-Feshbach Model}

\subsection{Nuclear Structure Data}

\subsubsection{Nuclear Masses and $J^{\pi}$ Assignments}

We adopt for nuclear masses the experimental mass excess values of (Möller et al. 1995). Spin and parity assignments are from the Evaluated Nuclear Structure Data File (ENSDF 2003). In Appendix 10 we present the binding energies (in $\mathrm{MeV}$ ) calculated from the adopted masses, the ground state spins and parities, and the separation energies for neutrons, protons, alpha-particles, and deuterons for the nuclei included in this study. In Appendix 11, we provide reaction Q-values for various cross sections that were calculated in this study. 


\subsubsection{Nuclear Level Schemes}

The nuclear structure data needed to model the gamma-ray cascade in this study was adopted from the file BUDAPEST.DAT (RIPL 1998). For the samarium, europium and gadolinium isotopes, additional evaluation was performed by $\mathrm{R}$. Bauer (Bauer 2001). Shown in Appendix A.4 are the modified adopted nuclear levels schemes, indicating the adopted level energy, spin and parity assignments, and their gamma-cascade branching ratios. The number of excited levels adopted for each nucleus is given as the quantity " $\mathrm{N}$ " in Table 12 (we always include a ground state, i.e. when $\mathrm{N}=0$ only the ground state is included). For the unmodified isotopes, this was the number for which energy spin and parity were unambiguously assigned in the BUDAPEST file.

\subsection{Transmission Coefficients}

\subsubsection{Transmission Coefficients for Particles}

Our modeling effort includes reactions with incident neutrons, protons, alpha-particles, and deuterons. For neutrons and protons, we develop our own optical model for deformed rare-earth nuclei. For alphas and deuterons, we adopt well established shperical optical models (see below).

\subsubsection{Considerations Regarding Collectivity and Nuclear Deformations}

Our region of interest extends from the closed neutron shell $(\mathrm{N}=82)$ to the mid-shell region $(\mathrm{N}=96)$ of the rare-earth nuclei, where the shapes of the nuclei change from spherical to highlydeformed over a very narrow range in $\mathrm{N}$. In the former, spherical optical models are often quite accurate, but as one proceeds to the later where the shell model is either intractable or unreliable, one must often use geometrical, or collective models that assign specific shapes to the nuclei and examine the rotations or vibrations of such nonspherical shapes.

To gauge the onset of collective effects we appeal to nuclear systematics. One such measure of "collectivity" is the ratio vs. $\mathrm{N}$ of the energy of the first $J^{\pi}=4^{+}$excited state to the first $J^{\pi}=2^{+}$ excited state in even- $\mathrm{Z}$ even-N nuclei (Figure 1 ). The magic closed shell nuclei $(\mathrm{N}=82)$ exhibt collectivity near 1.6. Deformed vibrators occur between $2.0-2.4(84 \leq \mathrm{N} \leq 88)$, followed by transitional nuclei nuclei up to $3.3(\mathrm{~N} \geq 92)$, where a true rotational character is evident. The loaded ${ }^{151,153} \mathrm{Eu}$ targets sit right in the transition zone.

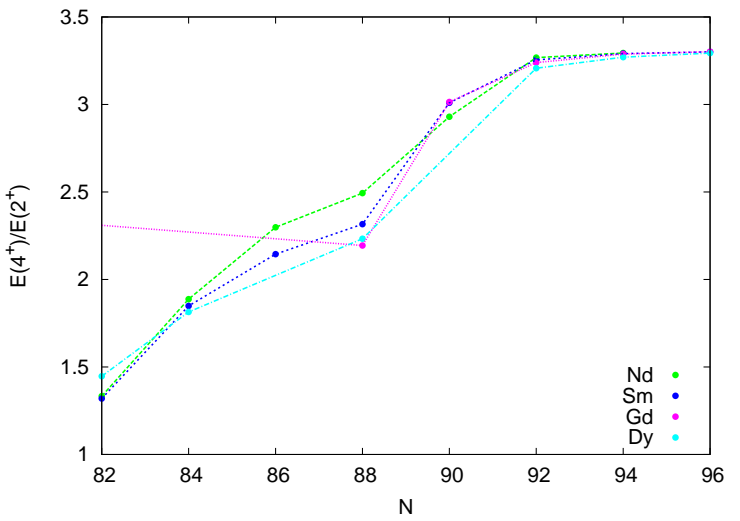

Fig. 1.- Collectivity in the region of $\mathrm{Sm}, \mathrm{Eu}$, and Gd.

Table 3: Adopted $\beta$ deformation parameters.

\begin{tabular}{cccccc}
\hline \hline $\mathrm{N}$ & $\mathrm{Pm}$ & $\mathrm{Sm}$ & $\mathrm{Eu}$ & $\mathrm{Gd}$ & $\mathrm{Tb}$ \\
\hline 81 & $.045^{*}$ & $-.053^{*}$ & $-.061^{*}$ & $-.053^{*}$ & $-.070^{*}$ \\
82 & $.000^{*}$ & .085 & .081 & .008 & $-.008^{*}$ \\
83 & $-.035^{*}$ & .097 & .094 & .091 & $-.052^{*}$ \\
84 & $.000^{*}$ & .120 & .119 & .117 & $-.044^{*}$ \\
85 & $.153^{*}$ & .126 & .123 & .120 & $.143^{*}$ \\
86 & $.161^{*}$ & .133 & .128 & .124 & $.161^{*}$ \\
87 & $.189^{*}$ & .142 & .139 & .135 & $.188^{*}$ \\
88 & $.216^{*}$ & .160 & .133 & .171 & $.216^{*}$ \\
89 & $.224^{*}$ & .182 & .181 & .175 & $.216^{*}$ \\
90 & $.251^{*}$ & .241 & .264 & .249 & $.243^{*}$ \\
91 & $.261^{*}$ & .251 & .248 & .246 & $.252^{*}$ \\
92 & $.270^{*}$ & .265 & $.270^{*}$ & .263 & $.271^{*}$ \\
93 & $.270^{*}$ & $.270^{*}$ & $.270^{*}$ & $.271^{*}$ & $.271^{*}$ \\
94 & $.279^{*}$ & $.279 *$ & $.270^{*}$ & $.271^{*}$ & $.271^{*}$ \\
95 & & & $.279^{*}$ & $.280^{*}$ & $.271^{*}$ \\
96 & & & $.280^{*}$ & $.280^{*}$ & \\
97 & & & $.290^{*}$ & & \\
\hline
\end{tabular}

* $\beta$ parameter taken from the mass tables of (Möller et al. 1995).

We adopt a deformed optical model for all our calculations, assuming a vibrational character for all nuclei up to $\mathrm{N}=88$, and a rotational one for nuclei above this (see below). We also adopt the deformation parameters $(\beta)$ listed in Table (3.2.2). Values marked with an asterisk were taken from the FDRM calculation of (Möller et al. 1995).

\subsubsection{The Neutron and Proton Optical Potential}

The optical potential used in determining the neutron and proton transmission coefficients was developed by F. Dietrich (Dietrich 2001). It is a 
deformed potential with a standard Woods-Saxon shape. The real volume potential has a depth of

$$
\begin{aligned}
V_{\text {vol. }}(E) & =(50.125-0.2331 E) \\
& -\eta_{p} \eta_{t}(20.050-0.0933 E) \\
& +\xi \frac{0.3 Z}{A^{1 / 3}}
\end{aligned}
$$

where

$$
\eta_{t}=\frac{A-2 Z}{A}
$$

and $\eta_{p}=1$ for incident neutrons and $\eta_{p}=-1$ for incident protons. Additionally, $\xi=1$ for incident neutrons and $\xi=0$ for incident protons. The real volume potential has a mean radius and diffusivity of $r_{v o l}=1.25 A^{1 / 3}$ and $a_{v o l} .=0.65$, respectively.

The imaginary volume potential has a depth of

$$
\begin{aligned}
W_{\text {vol. }}(E) & =(-1.357+0.1696 E) \\
& -\eta_{p} \eta_{t}(-0.543+0.0678 E)
\end{aligned}
$$

When $W_{v o l}$. $<0$, it is set to zero (i.e. we do not allow $W_{\text {vol }}$. to become negative). The mean radius and diffusivity are the same as for the real volume potential.

The imaginary surface potential is broken into two parts. For incident energies less than $8 \mathrm{MeV}$, it has the form

$$
\begin{aligned}
W_{\text {surf. }}(E) & =(3.743+0.334 E) \\
& -\eta_{p} \eta_{t}(1.497+0.134 E)
\end{aligned}
$$

Above $8 \mathrm{MeV}$, we use

$$
\begin{aligned}
W_{\text {surf. }}(E) & =(6.974-0.0697 E) \\
& -\eta_{p} \eta_{t}(2.790-0.0279 E)
\end{aligned}
$$

As with the imaginary volume, we only use positive values for the surface potential, replacing negative values with zero. The mean radius is the same as for the volume terms, but the diffusivity is taken to be $a_{\text {surf. }}=0.58$.

Last of all we include a real spin-orbit potential with a depth of $8.427 \mathrm{MeV}$ with the same mean radius and diffusivity as the volume terms. Additionally, for incident protons, one must include the Coulomb potential.

The particle transmission coefficients were generated by the optical model code ECIS-95 (Raynal 1996), using a deformed potential. For $N<88$ we used a vibrational model, and for $N \geq 88$ we used a rotational model. For vibrational cases, we use a one-phonon model. For
Table 4: Experimental vs. calculated total neutron cross sections at $14.2 \mathrm{MeV}$.

\begin{tabular}{lcc}
\hline \hline Target & Exp. $\sigma_{\text {tot }}$ (barns) & Calc. $\sigma_{\text {tot }}($ barns $)$ \\
\hline${ }^{149} \mathrm{Sm}$ & $5.07 \pm 0.04$ & 5.0775 \\
${ }^{150} \mathrm{Sm}$ & $5.36 \pm 0.04$ & 5.1612 \\
${ }^{152} \mathrm{Sm}$ & $5.54 \pm 0.04$ & 5.2176 \\
${ }^{154} \mathrm{Sm}$ & $5.59 \pm 0.04$ & 5.2638 \\
${ }^{154} \mathrm{Gd}$ & $5.23 \pm 0.08$ & 5.2289 \\
${ }^{155} \mathrm{Gd}$ & $5.34 \pm 0.07$ & 5.2427 \\
${ }^{156} \mathrm{Gd}$ & $5.42 \pm 0.04$ & 5.2714 \\
${ }^{157} \mathrm{Gd}$ & $5.27 \pm 0.04$ & 5.2945 \\
${ }^{160} \mathrm{Gd}$ & $5.48 \pm 0.03$ & 5.3514 \\
\hline
\end{tabular}

rotational cases, we allow up to quadrupole deformations. In either case, we include only one or two excited states. The states used are the ground state and first $J^{\pi}=2^{+}, 4^{+}$for even- $\mathrm{Z}$ even-N nuclei. For the remaining nuclei, a fictitious $0^{+}, 2^{+}$, $4^{+}$level scheme was developed. The energies for the levels were found by averaging the energies of the nearest even- $\mathrm{Z}$ even- $\mathrm{N}$ nuclei.

\subsubsection{Evaluation of the Neutron and Proton $\mathrm{Op}$ - tical Potential}

We present in Figure 2 results of the optical model compared to measured total neutron cross sections. The comparisons in Figure 2 are for total neutron cross sections on ${ }^{144} \mathrm{Sm}$ and ${ }^{148} \mathrm{Sm}$. Other experimental total neutron cross section data in this region generally consists of a single point at roughly $14 \mathrm{MeV}$, or several points below $10 \mathrm{keV}$ of excitation energy. For cases with a single data point at $14.2 \mathrm{MeV}$, the optical model closely replicated the experimental data, as summarized in Table (4). No total proton cross section data was available in this region.

The comparison between the calculated total neutron cross sections from our optical potential and available experimental data indicates a reasonable degree of agreement.

One can also calculate expected values for sand p-wave strength functions directly from the optical potential. In Figure 3 we provide a comparison between the values of these quantities as predicted by our optical potential against experimental data from (RIPL 1998), and Mughabghab et al. $(1973,1982)$. Generally, the optical potential replicated the s-wave strength functions reasonably well. The predicted values of the p-wave strength function are not quite so good, but are reasonable values. 

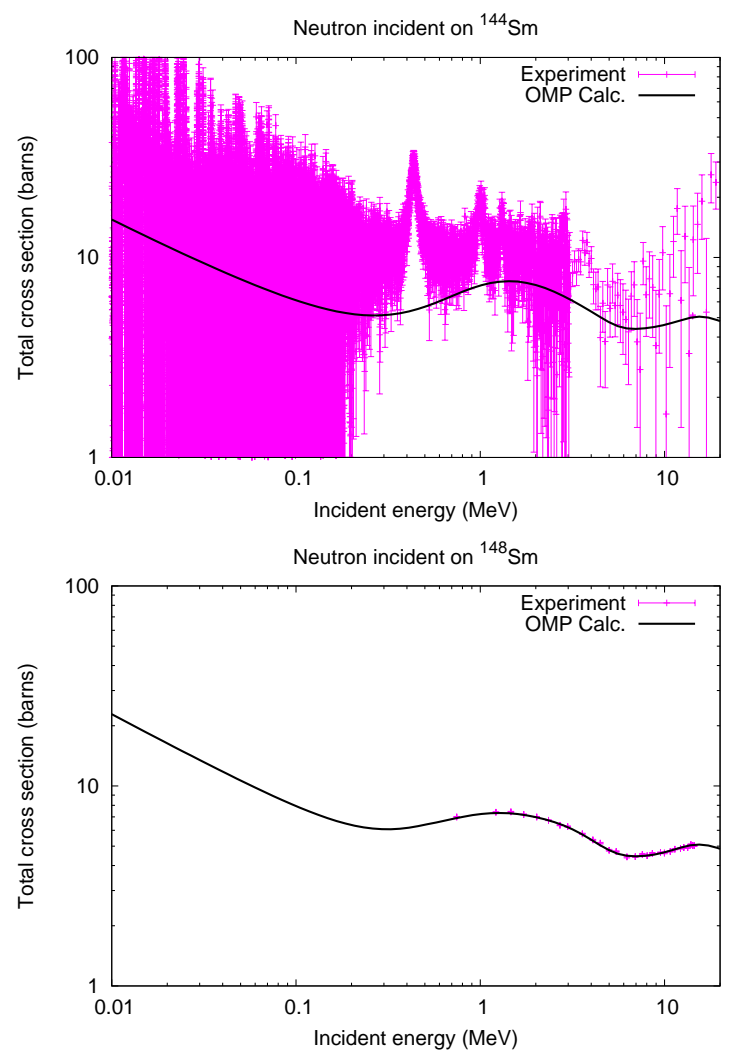

Fig. 2.- Total measured neutron cross sections vs. Optical Potential for ${ }^{144} \mathrm{Sm}+\mathrm{n}$ and ${ }^{148} \mathrm{Sm}+\mathrm{n}$.

Last of all, we present in Figure 4, the values for the mean scattering radius $\left(R^{\prime}\right)$ as predicted by our optical model. The predicted values are compared with the experimental values from Mughabghab et al. (1973, 1982). In general, the predicted values are reasonable, with particularly good agreement with the data for gadolinium isotopes. The choice of $x$-axis $(\mathrm{A}+5 \mathrm{Z})$ is only intended to spread out the $\mathrm{Sm}, \mathrm{Eu}$, and Gd points for purpose of illustration.

\subsubsection{The Alpha and Deuteron Optical Poten- tials}

We have included possible alpha and deuteron exit channels (and appropriate transmission coefficients) in this modeling effort. For the alpha particles, we use the optical potential of (Avrigeanu et al. 1994), for deuterons we use (Lohr \& Haeberli 1974), as encoded in the spherical optical model subroutine SCAT2 of STAPRE.

We do not include a quality analysis of these potentials in this report. The deuteron and alpha exit channels are, in every case, very small when compared to the dominant channel, account-
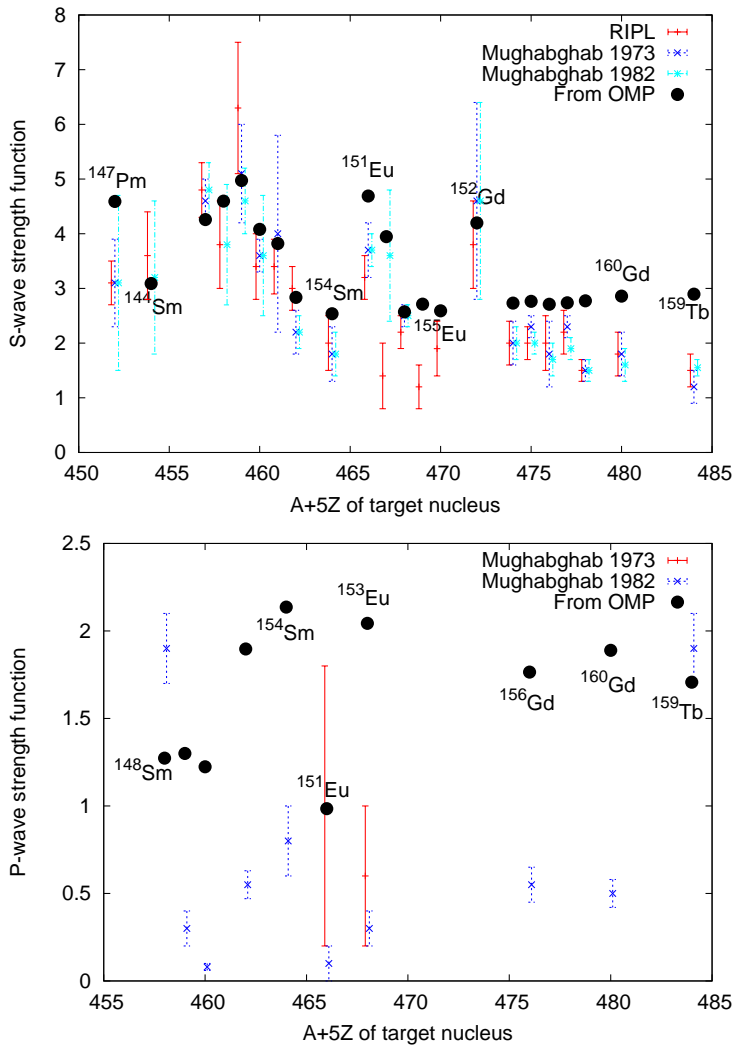

Fig. 3.- Comparison of predicted and experimental s- and p-wave strength functions.

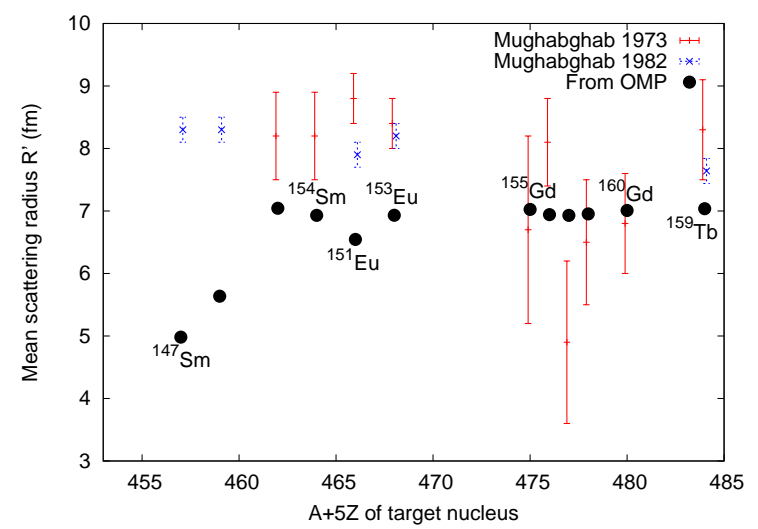

Fig. 4.- Comparison of predicted and experimental mean scattering radii.

ing for at most $1 \%$ of the total reaction cross section. Therefore, any sensitivity to the alpha and deuteron potentials will only be apparent in these weak exit channels which will have little or no impact on the analysis of UGT shots. Additionally, the relatively good agreement with the experimental $(\mathrm{n}, \alpha)$ cross sections provides us with some degree of confidence in the alpha potential (see $\S \mathrm{B}$ ). 


\subsubsection{Transmission Coefficients for Photons}

Gamma ray transmission coefficients were calculated using a simple model which depends only on the multi-pole type (XL) and the transition energy $(\epsilon)$, as encoded in STAPRE. They are related to the gamma ray strength function $f_{X L}^{\gamma}(\epsilon)$ by

$$
T_{X L}^{\gamma}(\epsilon)=2 \pi \epsilon^{2 L+1} f_{X L}^{\gamma}(\epsilon)
$$

The energy dependence of the strength function was determined using the GDR model with enhanced generalized Lorentzian (EGLO) line shapes (Kopecky et al. 1993). In particular, the E1 strength function is given by

$$
\begin{aligned}
& f_{E 1}^{\gamma}(\epsilon)=\mathcal{N} \frac{4}{3 \pi} \frac{e^{2}}{\hbar c} \frac{1}{M_{p} c^{2}} \times \\
& {\left[\frac{\epsilon \Gamma_{G D R}\left(\epsilon, T_{f}\right)}{\left(\epsilon^{2}-E_{G D R}^{2}\right)^{2}+\left(\Gamma_{G D R}\left(\epsilon, T_{f}\right) \epsilon\right)^{2}}+\right.} \\
& \left.0.7 \frac{\Gamma_{G D R}\left(0, T_{f}\right)}{\epsilon^{3}}\right]
\end{aligned}
$$

where $M_{p}$ is the proton mass. The energy dependent width $\Gamma_{G D R}\left(\epsilon, T_{f}\right)$ is given by

$$
\begin{aligned}
\Gamma_{G D R}\left(\epsilon, T_{f}\right)= & {\left[\kappa+(1-\kappa) \frac{\epsilon-\epsilon}{E_{G D R}-\epsilon}\right] \times } \\
& \frac{\Gamma_{G D R}}{E_{G D R}^{2}}\left[\epsilon^{2}+\left(2 \pi T_{f}\right)^{2}\right]
\end{aligned}
$$

with $\epsilon=4.5 \mathrm{MeV}$. For nuclei with $A<148$, the factor $\kappa$ is unity. For heavier nuclei, $\kappa=$ $1+0.009(A-148)^{2} \exp [-0.18(A-148)]$. The $T_{f}$ that appears in Equations 9 and 10 is the temperature of the final state, determined from the level density parameters. For a back-shifted transition energy $U=S_{n}-\epsilon-\Delta$, one determines the energy dependent level density parameter (Eq. 24). Provided $U$ is positive, the temperature is given by

$$
T_{f}=\frac{a}{2}[1+\sqrt{1+4 a U}]
$$

Otherwise, $T_{f}=1 / a$. The GDR parameters are given by

$$
\begin{aligned}
E_{G D R} & =\frac{80 \mathrm{MeV}}{A_{C}^{1 / 3}} \\
\Gamma_{G D R} & =5 \mathrm{MeV} \\
\sigma_{G D R} & =A_{C} \frac{13 \mathrm{mb}}{5}
\end{aligned}
$$

where $A_{C}$ is the mass number of the compound nucleus. We use only one E1 resonance.
We also include M1, E2, and M2 transitions. For the M1 strength function, we adopt a Simple Lorentzian (SLO) model

$$
\begin{array}{r}
f_{M 1}^{\gamma}(\epsilon)=\mathcal{N}_{M 1} \frac{4}{3 \pi} \frac{e^{2}}{\hbar c} \frac{1}{M_{p} c^{2}} \times \\
\frac{\epsilon \Gamma_{G D R}}{\left(\epsilon^{2}-E_{G D R}^{2}\right)^{2}+\left(\Gamma_{G D R} \epsilon\right)^{2}}
\end{array}
$$

with the global set of GDR parameters given in (RIPL 1998):

$$
\begin{aligned}
E_{G D R} & =\frac{41}{A^{1 / 3}} \\
\Gamma_{G D R} & =4
\end{aligned}
$$

The overall normalization for the M1 strength function $\left(\mathcal{N}_{M 1}\right)$ is determined such that

$$
\frac{f_{E 1}\left(S_{n}\right)}{f_{M 1}\left(S_{n}\right)}=0.0588 \cdot A^{0.878}
$$

where $S_{n}$ is the neutron separation energy.

The E2 and M2 transmission coefficients are simply proportional to $\epsilon^{2 L+1}$, their strength functions are constants. In particular,

$$
\begin{aligned}
f_{E 2}^{\gamma}(\epsilon) & =7.2 \times 10^{-7} A_{C}^{2 / 3} f_{E 1}^{\gamma}\left(S_{n}\right) \\
f_{M 2}^{\gamma}(\epsilon) & =2.2 \times 10^{-7} f_{E 1}^{\gamma}\left(S_{n}\right)
\end{aligned}
$$

where $S_{n}$ is the neutron separation energy. In all cases, E1 is the dominant multipole.

The factor $\mathcal{N}$ appearing in equation 9 is a normalization constant, determined by fitting the average total s-wave radiation width at the neutron binding energy,

$$
\begin{gathered}
\left\langle\Gamma_{\gamma}\right\rangle_{0}=\frac{J+1}{2 J+1}\left\langle\Gamma_{\gamma}\left(B_{n}, J+\frac{1}{2}\right)\right\rangle \\
+\frac{J}{2 J+1}\left\langle\Gamma_{\gamma}\left(B_{n}, J-\frac{1}{2}\right)\right\rangle \\
\Gamma_{\gamma}(E, J)=\frac{T_{\gamma}(E, J)}{2 \pi \rho(E, J)}(\mathrm{meV})
\end{gathered}
$$

(Uhl \& Strohmaier 1976).

Here, $J$ is the spin of the target nucleus. The gamma-ray transmission coefficients are evaluated as in equation 3 . Since the total s-wave radiation width is generally measured only for stable isotopes plus a neutron, we developed a systematic approach for estimating this value for the many unstable nuclei in our region of interest. These are determined by a least squares linear fit to experimental data, with separate systematics developed 

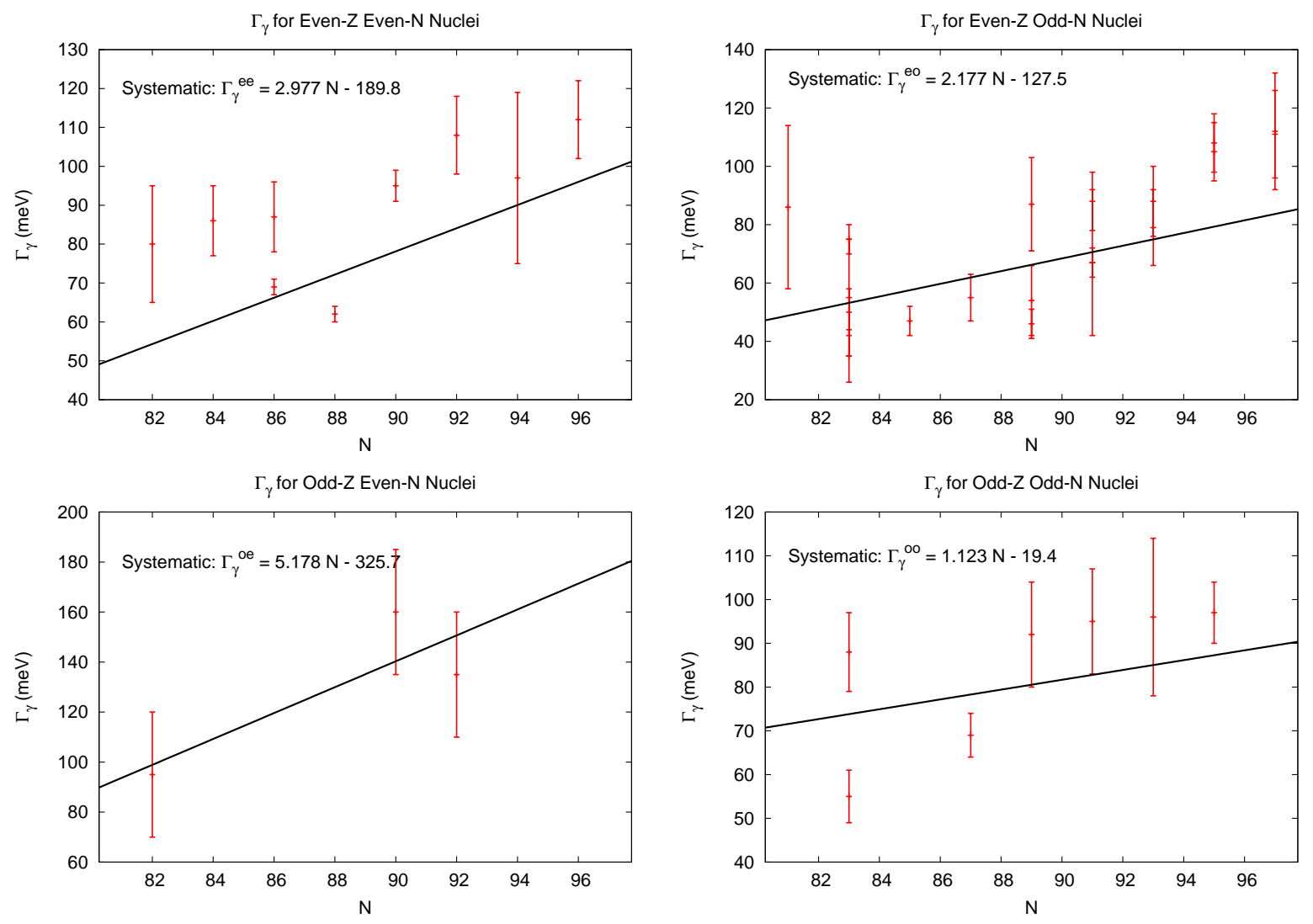

Fig. 5.- Systematics for average total s-wave radiation width.

for even-Z even-N, even-Z odd-N, odd-Z even-N, and odd-Z odd-n nuclei (Figure 5). We used the experimental values for all systems that had measured average s-wave radiation widths.

\subsection{Nuclear Level Densities}

\subsubsection{Level Density Models}

Another important input to the statistical model code, especially for the capture reactions, is the nuclear level density. For this project, we have adopted a standardized, semi-empirical approach (Gilbert \& Cameron 1965) which is numerically efficient, can be tied to experimental data, and is fairly accurate. The level density is described by two functions. Both are energy dependent, the second factor contains the spin dependence. This is the "Back-shifted Fermi Gas" formulation of the nuclear level density:

$$
\rho(U, J)=\rho(U) f(U, J)
$$

where $\rho(U)$ is the state density, with $U=E-\Delta$ the back-shifted energy. $\Delta$ is the so called "pairing energy", and $J$ is the spin of the compound nucleus. We will further treat each of these in two ways, depending on the excitation energy of interest. The demarcation point will be roughly between the energy range of the known excited levels of a given compound nucleus (the low energy domain), and near (and above) the neutron binding energy (the high energy domain).

For the high energy domain, we describe the level density assuming a Fermi gas formula,

$$
\begin{array}{r}
\rho(U)=\frac{\sqrt{\pi}}{12} \frac{\exp (2 \sqrt{a U})}{a^{1 / 4} U^{5 / 4}} \frac{1}{\sqrt{2 \pi} \sigma} \\
f(U, J)=\frac{2 J+1}{2 \sigma^{2}} \exp \left[\frac{-\left(J+\frac{1}{2}\right)^{2}}{2 \sigma^{2}}\right]
\end{array}
$$

where $a(E)$ is the level density parameter (in $\left.\mathrm{MeV}^{-1}\right)$. The spin cutoff parameter $\sigma^{2}$ is defined as

$$
\sigma^{2}=0.01496 \lambda A^{5 / 3} \sqrt{\frac{U}{a}}
$$

The level density assumes an equal distribution of parity states. Note that at low excitation energy (for a positive back-shift), Eq. [19] diverges. At 
low energies, the nuclear level density is better described by a constant temperature formula:

$$
\rho(E) \propto \exp \frac{E-E_{0}}{T}
$$

The level density parameters can be calculated using experimental data. For the Fermi-gas state density (Eq. [19]), the level density parameter, $a(E)$, can be related to the average level spacing $\left(D_{0}\right)$ near the neutron binding energy. The pairing energies used in the calculation of the back shifted energy are calculated as differences of binding energies (Bohr \& Mottelson). The constant temperature parameters $E_{0}$ and $T$, can be chosen to provide a state density that goes through the low lying spectroscopic levels subject to the choice of a matching energy, $E_{x}$, chosen someplace between the high and low energy regions of interest, at which the two state densities match (point and slope). We describe below how we determined these parameters for all of the nuclei considered in this study.

\subsubsection{Level Densities Above the Neutron Bind- ing Energy}

Our goal is to fit the level density parameter $a$ in Eq. [19] to experimental data where available. We adopt an energy dependent form, $a(U, Z, N)$, (Iljinov et al. 1992), and begin by fixing the spin cutoff parameter and the pairing energies.

\section{The Spin Cutoff Parameter}

The spin cutoff parameter $\sigma^{2}$, Eq. [21], characterizes the spin distribution of the Fermi gas level density. It depends on the parameters $a$, the level density parameter, and $\lambda$, which determines the effective moment of inertia for the nucleus in question. In principle it could be determined by experiment, for example, by comparing ratios of cross sections leading to different isomers of the product nucleus (Keisch 1963). Because data like this is often sparse, especially in the limited regions of the periodic chart we are interested in, and because we are often interested in reactions that proceed on or through radioactive species where no such data exists, we must resort to models. In our analysis, we fix $\lambda=1$ in Eq. [21], corresponding to the moment of inertia of a rigid sphere.

\section{Pairing Energies}

In determining the back-shift $\Delta$, also known as the pairing energy, we used a slightly modified version of the method of Rauscher (Rauscher et al. 1997). The total pairing energy is equal to the sum of the proton and neutron pairing energies

$$
\begin{aligned}
\Delta(Z, N) & =\frac{1}{2}\left(\Delta_{p}+\Delta_{n}\right) \\
\Delta_{p}(Z, N) & =E^{G}(Z, N) \\
& -\frac{1}{2} E^{G}(Z-1, N) \\
& -\frac{1}{2} E^{G}(Z+1, N) \\
\Delta_{n}(Z, N) & =E^{G}(Z, N) \\
& -\frac{1}{2} E^{G}(Z, N-1) \\
& -\frac{1}{2} E^{G}(Z, N+1)
\end{aligned}
$$

where $E^{G}(Z, N)$ is the binding energy of the nucleus $(Z, N)$. In calculating the binding energies of the various nuclei, we use the experimental mass excesses listed in the Möller and Nix tables (Möller et al. 1995).

\section{The Level Density Parameter}

At high energies, the level density parameter $a$ behaves essentially as a function of mass number only. However, in cases where deformation and shell effects are important (often the case near closed neutron shells) it has been shown (Iljinov et al. 1992) that at low energies it is more appropriate to use an energy dependent form of the level density parameter;

$$
a(U, Z, N)=\tilde{a}(A)\left[1+\delta W(Z, N) \frac{f(U)}{U}\right]
$$

with

$$
f(U)=1-\exp (-\gamma U)
$$

and as usual $U=E-\Delta$. In previous works where a more global prescription was developed [(Rauscher et al. 1997), (Iljinov et al. 1992)], one would adopt a semi-empirical shell correction, $\delta W(Z, N)$, and fit $\tilde{a}(A)$ to known experimental data. Here we choose to adopt a simple form for the mass dependent term and fit the shell correction. In our analysis, we followed the convention of (Rauscher et al. 1997) in choosing the parameters $\gamma=0.04884$ and $\tilde{a}=0.1337 A-0.06571 A^{2 / 3}$.

\section{Shell Corrections}

Shell corrections can be determined for select nuclei from experimental values of the average level 
spacing's $D_{0}$ as determined by neutron resonance analysis (RIPL 1998). For $s$-wave resonances (neutron angular momentum equal to zero), the calculated level spacing, $D_{\text {calc }}$, evaluated at the neutron binding energy $U=B_{n}$, is related to the nuclear level density (e.g. Eq.'s [18-20])

$$
D_{\text {calc }}=\frac{2}{\rho\left(U, J=\frac{1}{2}\right)}
$$

for nuclei with spin $s=0$ and

$$
D_{\text {calc }}=\frac{2}{\rho\left(U, J=s+\frac{1}{2}\right)+\rho\left(U, J=s-\frac{1}{2}\right)}
$$

for nuclei with $s \neq 0$. In each case, the level densities $\rho(U, J)$ were calculated using the other parameters $(\lambda, \Delta, \gamma, \tilde{a}(A))$ set as previously described. We then numerically solved for the value of $\delta W$ that would minimize the quantity $D_{\text {calc }}-$ $D_{0}$ using root bisection methods. This now completely describes the parameterization of $\sigma^{2}$ and $a(U, Z, N)$, and thus the Fermi-gas level density.

\subsubsection{Systematic Behavior of Fermi Gas Level Density Parameters}

There are only a limited number of nuclei for which the average resonance spacing $D_{0}$ has been measured (i.e. for compound nuclei formed from a stable target plus a neutron). As a result, we were required to systematically predict the shell correction for the remaining nuclei in the range of interest for this project. After plotting the experimental $\delta W$ in our region of interest and their associated errors we noted two segments with a roughly linear behavior. We made a $\chi^{2}$ linear fit to the data, shown in Figure 6 . Of course, where available, we always used an experimentally determined shell correction over a systematic one.

\subsubsection{Level Densities Below the Neutron Bind- ing Energy}

For the lower energy regions, below the neutron binding energy $B_{n}$, the nuclear level density has the same formulation as Eq. [18]. However, particularly at and below the pairing energy $\Delta$, the state density in Eq. [19] becomes imaginary. Unfortunately, experimental level schemes are rarely known above $2 \mathrm{MeV}$ of excitation energy. In practice we are forced again to assume a model and use all available experimental data to constrain its parameters.

Of course the two prescriptions for the level density must match at some energy intermediate to where they are constrained by experiment.

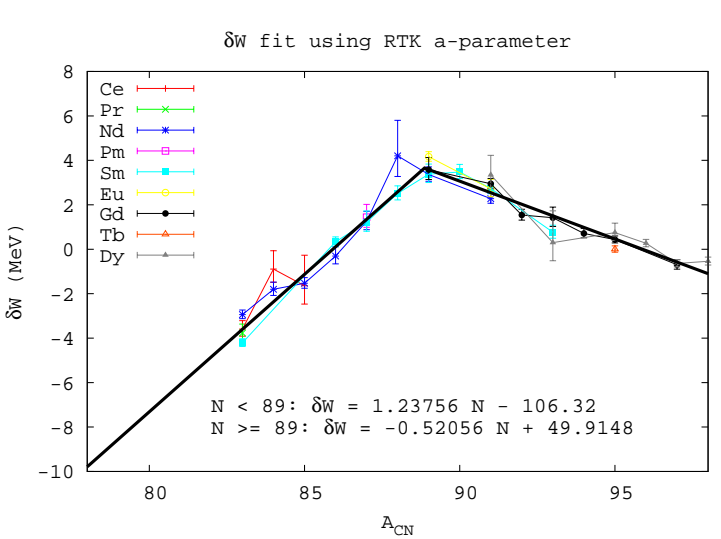

Fig. 6. - $\chi^{2}$ linear fit to experimentally determined shell corrections, used to systematically determine unknown shell corrections.

Henceforth we will refer to the high energy level density as $\rho_{1}$, and the low energy density as $\rho_{2}$.

Gilbert and Cameron (Gilbert \& Cameron 1965) noticed that the cumulative number of observed levels (the so-called staircase plot, which increase exponentially), can be fit with straight lines in a semi-log plot. They adopted a constant temperature formula to fit these:

$$
N(E)=\exp \left[\frac{E-E_{0}}{T}\right]
$$

with $N(E)$ being the cumulative number of levels at excitation energy $E, E_{0}$ and $T$ are two free parameters to be fit to the observed level structure. The observable level density is given by

$$
\rho_{1}(E)=\frac{d N(E)}{d E}=\frac{1}{T} \exp \left[\frac{E-E_{0}}{T}\right]
$$

From classical thermodynamics, we have a definition of the nuclear temperature

$$
\frac{d}{d E} \log \rho_{1}(E)=\frac{1}{T}
$$

where $T$ now takes on the meaning of a nuclear temperature which is constant in the region of the discrete levels. We assume that Eq. [28] can be extrapolated from the region of the known discrete levels to higher energies, where the Fermi-gas level density $\left(\rho_{1}\right)$ is valid. We then define the notion of a fit to the total level density over the entire range as being achieved if: a) a good fit can be made to the low lying levels, b) the observed level spacing at the neutron binding energy is exactly reproduced, and c) the energy of the matching point $E_{x}$ for the two prescriptions falls between $E=0$ and $E=$ 
$B_{n}$, and that they match at this point with the same slope, i.e. for $E=E_{x}$ :

$$
\begin{aligned}
\rho_{1}\left(E_{x}\right) & =\rho_{2}\left(E_{x}\right) \\
\frac{d \log \rho_{1}\left(E_{x}\right)}{d E} & =\frac{d \log \rho_{2}\left(E_{x}\right)}{d E}
\end{aligned}
$$

From the first of these, we can determine $E_{0}$ :

$$
E_{0}=E_{x}-T \log T \rho_{2}\left(U_{x}\right)
$$

where $U_{x}=E_{x}-\Delta$. The second condition can be satisfied by assuming that at $E_{x}$ the constant nuclear temperature $T$ of the low lying states is equal to the energy dependent nuclear temperature $\tau\left(U_{x}\right)$ of the high excited states,

$$
\begin{aligned}
\frac{1}{T} & =\sqrt{\frac{a}{U_{x}}}-\frac{3}{2 U_{x}} \\
& +\frac{(\tilde{a}-a)\left(1+\gamma U_{x}\right)+\tilde{a} \gamma \delta W}{\sqrt{a U_{x}}}
\end{aligned}
$$

where $a$ is given by Eq. [24]. If there is no shell correction, the latter term in the above equation is zero. Typical values for the matching energy are $2 \leq E_{x} \leq 8 \mathrm{MeV}$, and are approximated by $E_{x}=2.5+\frac{150}{A}+\Delta$ (Gilbert \& Cameron 1965). The constant temperature fits to the low lying levels of ${ }^{151,153} \mathrm{Eu}$ and ${ }^{151,153} \mathrm{Gd}$ are presented in Figure 7 .

Behavior of the Spin Cutoff Parameter Below $E_{x}$

At the matching energy $E_{x}$, the spin cutoff parameter is given by Eq. [21]. For energies below $E_{x}$, we assume $\sigma^{2}=\sigma^{2}\left(E_{x}\right)$.

Other treatments have been suggested for the behavior of $\sigma^{2}$ below $E_{x}$. One may define $E_{\text {cut }}$ as the energy of the highest known excited level for which energy, spin and parity are explicitly known. Using the known spectroscopic levels, a low energy spin cutoff parameter at $E_{c u t}$ may be defined:

$$
\sigma_{E_{c u t}}^{2}=\frac{1}{2 N} \sum_{i=1}^{N}\left(J_{i}+\frac{1}{2}\right)^{2}
$$

where $\mathrm{N}$ represents the number of the level with energy $E_{c u t}, J_{i}$ are the spins of the individual levels. The sum excludes the ground state $(\mathrm{i}=0)$. This value is used for energies $0 \leq E \leq E_{\text {cut }}$. For energies $E_{\text {cut }} \leq E \leq E_{x}$, the spin cutoff parameter is given by a linear fit between these two values (Eq.'s [21] and [35]). Specifically,

$$
\sigma_{I}^{2}=\sigma_{E_{x}}^{2}-\frac{\sigma_{E_{x}}^{2}-\sigma_{E_{c u t}}^{2}}{E_{x}-E_{c u t}}\left(E_{x}-E\right)
$$
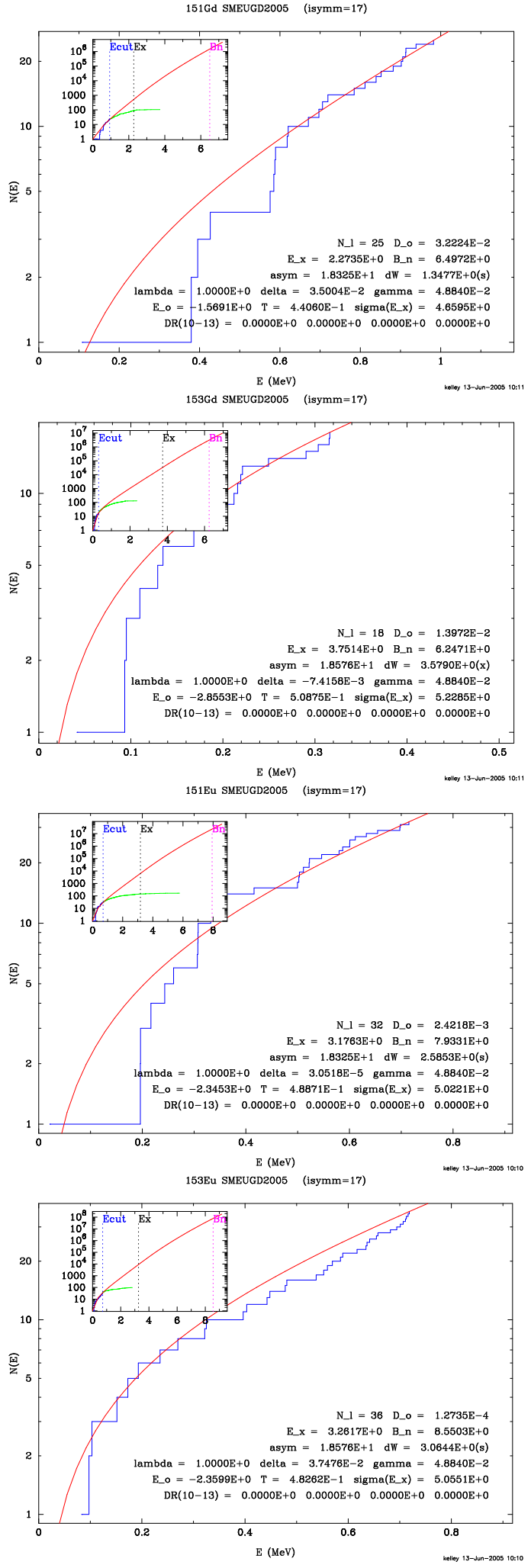

Fig. 7.- Constant temperature level density fits to the low lying spectroscopic levels of ${ }^{151,153} \mathrm{Eu}$ and ${ }^{151,153} \mathrm{Gd}$. 
This conforms to the treatment of $\sigma^{2}$ in the IDA reaction code system (Reffo 1978). This particular treatment works well when there is a reasonably large number of levels to fit.

Another treatment, used in the GNASH code system (Chadwick 1998), defines:

$$
\begin{aligned}
\sigma_{H}^{2} & =\sigma_{E_{x}}^{2} \\
U_{L} & =\max \left(E_{\text {cut }}-\Delta, 0.1\right) \\
\sigma_{L}^{2} & =\lambda \sqrt{a U_{L}} A^{2 / 3} \\
\sigma_{G}^{2} & =\sigma_{L}^{2}+\frac{E-\frac{1}{2} E_{c u t}}{E_{x}-\frac{1}{2} E_{c u t}}\left(\sigma_{H}^{2}-\sigma_{L}^{2}\right)
\end{aligned}
$$

The form $\sigma_{G}^{2}$ is then used between $\frac{1}{2} E_{c u t}$ and $E_{x}$.

The behavior of $\sigma^{2}$ below $E_{x}$ will only affect the level density used in Hauser-Feshbach calculations between $E_{c u t}$ and $E_{x}$, since the discrete levels are accounted for individually. The changes that arise between $E_{c u t}$ and $E_{x}$ are generally small.

The fitted parameters for the total level density are presented in Table 12. The symbols in the legend are the same as described above. In column five, an "x" indicates the shell correction $\delta W$ was derived from an experimentally known level spacing $D_{0}$, an "s" indicates the shell correction was derived from the systematic shown in Figure 6.

\subsection{Considerations Regarding the Exci- ton Model}

When including alpha particles as a possible exit channel, one must account for them in the pre-equilibrium phase of the reaction. In particular, they must be accounted for in the exciton model. Generally, the description of alpha particle emission in the pre-equilibrium model is a straightforward extension of the description of neutron or proton emission, given the tendency of nucleons to pre-form alpha clusters in the nucleus. In making such an extension, one introduces a parameter $\phi$ which represents the probability that the incoming particle will strike a pre-formed alpha cluster (Milazzo-Colli et al. 1973). It follows that the larger values of $\phi$ will result in a higher probability of subsequent alpha emission, thus enhancing the $(\mathrm{n}, \alpha)$ reactions.

In our calculations, we have chosen a value of $\phi=0.20$, although previous considerations of alpha emission suggest that this value may fall anywhere in the range of $0.1 \leq \phi \leq 0.8$ within the mass range of interest (Milazzo-Colli et al. 1973). We have used our chosen value primarily because it results in $(\mathrm{n}, \alpha)$ cross sections which best fit the available experimental data.
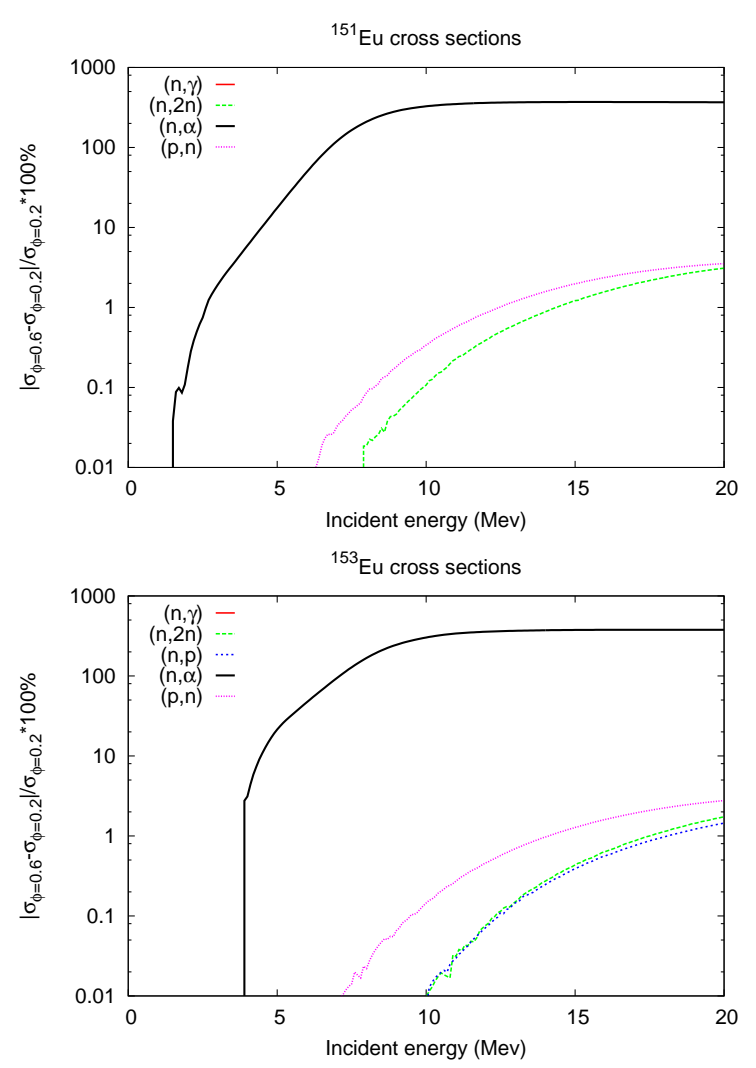

Fig. 8.- Sensitivity of ${ }^{151,153} \mathrm{Eu}(\mathrm{n}, 2 \mathrm{n}),(\mathrm{n}, \gamma)$, $(n, p)$ and $(p, n)$ cross sections to the alpha preformation parameter $\phi$

Since the alpha particle emission accounts for only a very small portion of the total reaction cross section (generally less than $1 \%$ ), variations in the $\phi$ parameter will only have minimal, if not negligible, effects on the other cross sections. We verified this result in the cases of $(n, 2 n),(n, \gamma),(n, p)$ and $(\mathrm{p}, \mathrm{n})$ reactions on stable europium targets Figure 8 shows the percentage difference of the cross sections calculated with $\phi=0.2$ and 0.6 . The $(\mathrm{n}, \alpha)$ cross sections are enhanced by nearly $400 \%$, while the $(\mathrm{n}, 2 \mathrm{n}),(\mathrm{n}, \mathrm{p})$ and $(\mathrm{p}, \mathrm{n})$ cross sections are reduced by at most one or two percent (note that the quantity given in the plot is the absolute percentage difference). The reduction in the $(n, \gamma)$ cross sections was in both cases less than $0.01 \%$ over the entire energy range. 


\section{Calculated Cross Sections}

\subsection{Comparison to Measured Cross Sec- tions}

Having developed the various input quantities based on available experimental data in the previous section, we now turn to results of the STAPRE-H95 model and compare to available experimentally measured cross sections in the region of interest. Results presented in this section are restricted to reactions on the two stable (loaded) ${ }^{151,153} \mathrm{Eu}$ targets, although many more comparisons are provided in the Appendices. Only results for targets initially in their ground states are available.

In Figure 9 we present the comparison for $(n, \gamma)$, $(\mathrm{n}, 2 \mathrm{n}),(\mathrm{p}, \mathrm{n})$, and $(\mathrm{d}, 2 \mathrm{n})$ reactions on ${ }^{151,153} \mathrm{Eu}$. These comprise the main destruction reactions for the loaded detector element, the charged-particle reactions are the principle production reactions for ${ }^{151,153} \mathrm{Gd}$. Shown is the activation cross section (solid black lines in all plots that follow) defined as the sum of emission (both particle emission and gamma-ray cascade) from the compound nucleus that eventually leads to the ground state of the product (final) nucleus. We also provide (where appropriate) separate cross sections that decay to the ground state (red lines), and any long lived isomer (blue and green lines, see Appendix 9 for a list of the isomers and their respective half-lives). These cross sections are plotted against the available experimental data, taken from the Experimental Nuclear Reaction Data File (CSISRS 2003) for the $(n, \gamma)$ and $(n, 2 n)$ reactions. For the $(\mathrm{p}, \mathrm{n})$ and $(\mathrm{d}, 2 \mathrm{n})$ reactions, the data is from (Nethaway \& Mustafa 1999). Cross sections for the total, ground, and isomeric states are colored in a similar manner to the modeled cross sections (gray is activation, orange is to ground, and light blue and green to an isomer, respectively), with different symbols distinguishing results from various experiments.

\subsubsection{Comparison to experimental $(n, \gamma)$ capture cross sections}

For ${ }^{151} \mathrm{Eu}(\mathrm{n}, \gamma){ }^{152} \mathrm{Eu}$, our result is in excellent agreement with the data between $10-200 \mathrm{keV}$, at least for the activation and amounts to the ground state and first isomer. Above $200 \mathrm{keV}$, our activation and ground state cross section still show good agreement with the data, but the first isomer cross section is low by roughly $50 \%$. Below $10 \mathrm{keV}$, our activation cross section is also some- what low. There is only limited data for the cross section to the second isomer. This is a good result for an $(n, \gamma)$ activation cross section. Using global systematics, $(n, \gamma)$ cross sections can typically be modeled within a factor of two, often to within 30\% (Hoffman et al. 1999).

The ${ }^{153} \mathrm{Eu}(\mathrm{n}, \gamma){ }^{154} \mathrm{Eu}$ activation cross section shows good agreement over the entire energy range of interest. It should be noted that there is no experimental data available on the production of the isomeric states, and the isomeric states of ${ }^{154} \mathrm{Eu}$ were not included in the previous RADCHEM databases (Eu0988 and Eu0289).

Additional comparison to experimental neutron capture cross sections for select Sm and Gd targets are given in Appendix B. Figure 21 shows $(\mathrm{n}, \gamma)$ cross sections that exhibit similar agreement (within $\sim 50 \%$ ) to the ${ }^{151,153} \mathrm{Eu}$ capture reactions. The agreement was not quite as good for gadolinium targets with an even number of neutrons, but overall the agreement is about as good as can be expected for a modeled $(n, \gamma)$ cross section. Overall we believe we have well predicted the measured $(n, \gamma)$ cross sections in the entire region of interest, and we expect our calculated capture cross sections on non-stable isotopes to have similar accuracy.

A quantitative comparison of our calculated cross sections to the experimental data shown in Figures 9 and 21 is given in Table 5. For $(n, \gamma)$ reactions we restrict our analysis to data with incident neutron energies of $30 \pm 2 \mathrm{keV}$. For each target listed in column 1), the subsequent column entries identify: 2) Res., the state of the residual (product) nucleus (activation, ground state, isomer); 3) $\mathrm{N}$, the number of experimental data points falling within the incident energy range; 4) $\bar{\sigma}$, the weighted average of the measured data (in barns), with weights corresponding to the inverse geometric mean of the errors in cross section and energy (i.e. $\left.w_{i}=\left(d E^{2}+d \sigma^{2}\right)^{-1 / 2}\right)$; 5) Dev., the standard deviation from the mean (also in barns), which gives an indication of the spread in the experimental data; 6) $\sigma_{\text {mod. }}$, our modeled capture cross section (in barns); and 7) $\bar{\sigma} / \sigma_{\text {mod. }}$, the ratio of the weighted average of the data to our modeled value, which may be used as a scaling factor to be applied to a given cross section to bring it into conformity with its respective average experimental value.

If one excludes the ${ }^{151} \mathrm{Eu}$ cross section going to the second isomer (for which there is only a single data point), our modeled results for $(n, \gamma)$ 

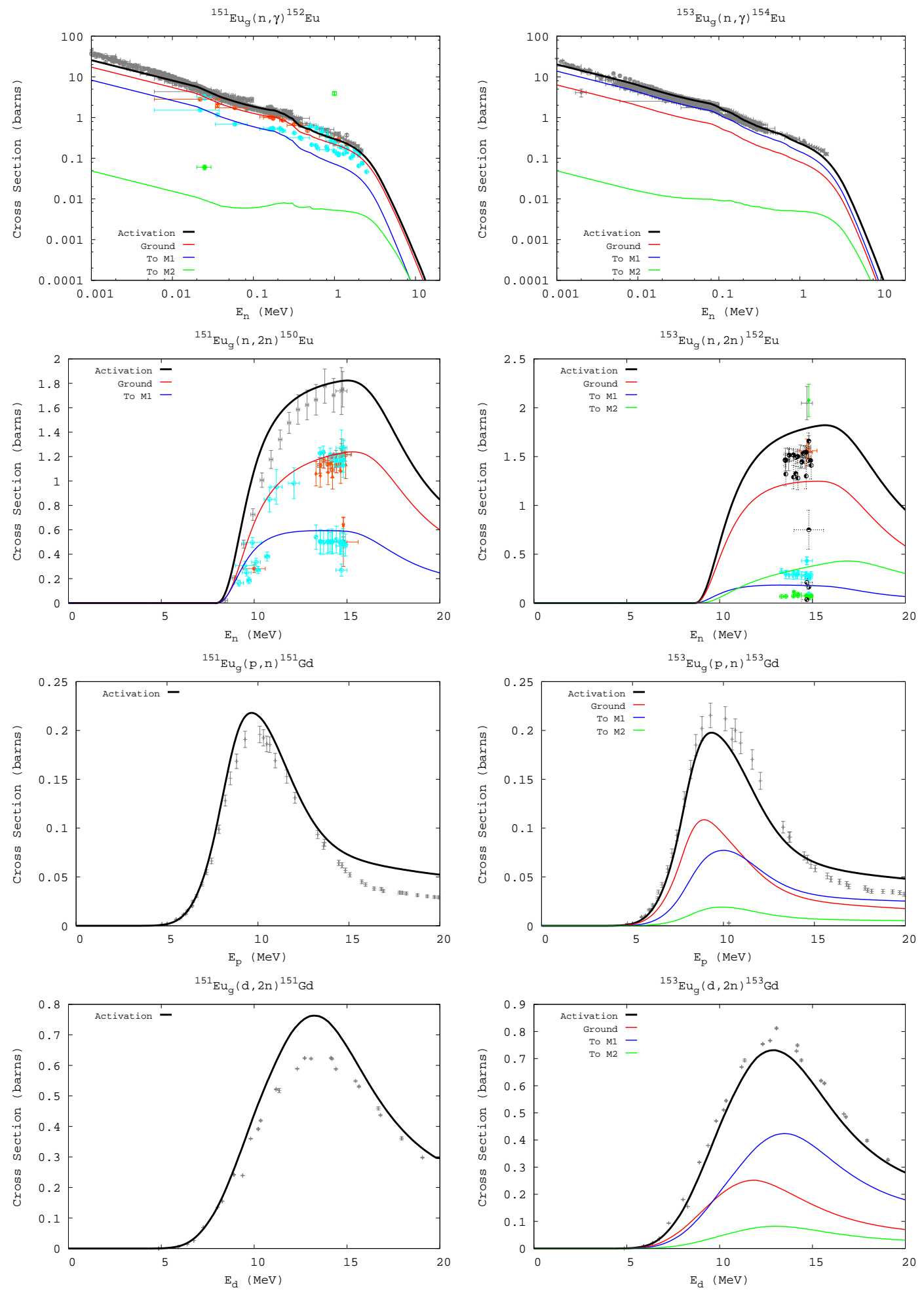

Fig. 9.- Calculated vs. experimental (n, $\gamma),(n, 2 n),(p, n)$ and (d,2n) cross sections: ${ }^{151,153} \mathrm{Eu}$.

cross sections are on average within $40 \%$ of the weighted mean value. Only ${ }^{160} \mathrm{Gd}$ differs by more than a factor of two. Considering only activa- tion (A) measurements, half of our calculated cross sections are within the standard deviation of the mean of the errors. If one further restricts atten- 
Table 5: Comparison of our modeled $(\mathrm{n}, \gamma)$ cross sections to experimental data at $30 \pm 2 \mathrm{keV}$

\begin{tabular}{lllllll}
\hline \hline${ }^{A} Z$ & Res. & $\mathrm{N}$ & $\bar{\sigma}$ & Dev. & $\sigma_{\text {mod. }}$ & $\bar{\sigma} / \sigma_{\text {mod }}$ \\
\hline${ }^{144} \mathrm{Sm}$ & $\mathrm{A}$ & 5 & 0.077 & 0.020 & 0.088 & 0.871 \\
${ }^{147} \mathrm{Sm}$ & $\mathrm{A}$ & 16 & 0.929 & 0.588 & 1.220 & 0.762 \\
${ }^{148} \mathrm{Sm}$ & $\mathrm{A}$ & 7 & 0.223 & 0.026 & 0.313 & 0.710 \\
${ }^{149} \mathrm{Sm}$ & $\mathrm{A}$ & 23 & 1.665 & 0.758 & 1.923 & 0.866 \\
${ }^{150} \mathrm{Sm}$ & $\mathrm{A}$ & 7 & 0.378 & 0.095 & 0.624 & 0.605 \\
${ }^{152} \mathrm{Sm}$ & $\mathrm{A}$ & 11 & 0.463 & 0.095 & 0.583 & 0.794 \\
${ }^{154} \mathrm{Sm}$ & $\mathrm{A}$ & 6 & 0.316 & 0.132 & 0.376 & 0.840 \\
${ }^{151} \mathrm{Eu}$ & $\mathrm{GS}$ & 1 & 2.822 & & 2.730 & 1.034 \\
${ }^{151} \mathrm{Eu}$ & $\mathrm{M} 1$ & 2 & 1.804 & 1.007 & 1.322 & 1.365 \\
${ }^{151} \mathrm{Eu}$ & $\mathrm{M} 2$ & 1 & 0.060 & & 0.008 & 7.320 \\
${ }^{151} \mathrm{Eu}$ & $\mathrm{A}$ & 37 & 3.561 & 0.675 & 4.060 & 0.877 \\
${ }^{153} \mathrm{Eu}$ & $\mathrm{A}$ & 38 & 2.612 & 0.426 & 3.356 & 0.778 \\
${ }^{152} \mathrm{Gd}$ & $\mathrm{A}$ & 4 & 0.950 & 0.057 & 0.922 & 1.031 \\
${ }^{154} \mathrm{Gd}$ & $\mathrm{A}$ & 7 & 0.888 & 0.246 & 1.282 & 0.693 \\
${ }^{155} \mathrm{Gd}$ & $\mathrm{A}$ & 8 & 2.704 & 0.303 & 2.935 & 0.921 \\
${ }^{156} \mathrm{Gd}$ & A & 4 & 0.587 & 0.024 & 0.898 & 0.653 \\
${ }^{157} \mathrm{Gd}$ & A & 8 & 1.406 & 0.133 & 1.667 & 0.844 \\
${ }^{158} \mathrm{Gd}$ & A & 11 & 0.354 & 0.139 & 0.546 & 0.648 \\
${ }^{160} \mathrm{Gd}$ & A & 9 & 0.145 & 0.081 & 0.319 & 0.455 \\
\hline \multicolumn{6}{c}{ Average error: } & $37.8 \%$ \\
& Average error (activation only): & $37.7 \%$ \\
\hline
\end{tabular}

tion to species with $82<\mathrm{N}<92$ (i.e. the region where the radioactivities of interest to radchem lie, and for which we developed our systematics), our results are within the errors $70 \%$ of the time.

As seen in previous modeling efforts on $\mathrm{Br}-\mathrm{Kr}$ and I-Xe, our modeled cross sections on even-Z even- $\mathrm{N}$ targets in this region are often higher than experiment suggests (true for five out of the seven activation cases that are outside the errors). We often do better on capture reactions proceeding on odd-N targets. This is likely an effect of our level density treatment, to be explored in the next section.

We also note the large spread in the cross sections associated with ${ }^{151,153} \mathrm{Eu}$ targets, an unacceptable situation for such a well studied pair of reactions. For ${ }^{153} \mathrm{Eu}$, the spread is so large that reasonable variations in our input systematics could provide cross section values that stay within the range of the measurements!

\subsubsection{Comparison to Maxwellian averaged $(n, \gamma)$ capture cross sections}

Yet another comparison to experimental data comes from the extensive efforts to measure and evaluate Maxwellian averaged capture cross sec- tions for astrophysical applications (Bao et al. 2000). The Maxwellian-averaged neutron capture cross section is defined as the reaction rate $\langle\sigma v\rangle$ divided by the mean velocity $v_{T}=\sqrt{2 k T / \mu}$ at a given temperature $T$. Here, $\mu$ is the reduced mass. For particle fluences and temperatures typical to stellar nucleosynthesis, the velocity distribution of the neutrons reduces to a Maxwell-Boltzmann distribution. In this case, the Maxwellian-averaged cross section reduces to (Beer et al. 1992)

$$
\begin{aligned}
\frac{\langle\sigma v\rangle}{v_{T}} & =\frac{\int_{0}^{\infty} \sigma_{n \gamma} v \Phi(v) d v}{v_{T}} \\
& =\frac{2}{\sqrt{\pi}(k T)^{2}} \int_{0}^{\infty} \sigma_{n \gamma}(E) W(E, k T) d E
\end{aligned}
$$

where $W(E, k T)=E \exp (-E / k T)$ and $E$ is the center of mass energy.

Figure 22 compares our calculated Maxwellianaveraged capture cross sections to their evaluated counterparts (Bao et al. 2000). The error bars on all points are identical and represent the measured error for a given cross section at $30 \mathrm{keV}$. We used spline interpolation to determine the value of the $(\mathrm{n}, \gamma)$ cross section between points on the energy grid. For energies below our lowest grid energy, we assume an $(\mathrm{n}, \gamma)$ cross section with an $E_{l a b}^{-1 / 2}$ dependence. For energies greater than our highest grid energy, we take the cross section to be zero.

Overall our calculated Maxwellian averaged cross sections agree with those of (Bao et al. 2000). Our results for a few targets tend to be high, but in a manner consistent with our comparison to other $(\mathrm{n}, \gamma)$ cross sections.

The greatest leverage in neutron capture calculations is due to normalization of the photon transmission coefficient $(\mathcal{N}$ in Eq. 9). Previous astrophysical modeling efforts have normalized their photon transmission coefficients to reproduce Maxwellian-averaged neutron capture cross sections rather than using measured average S-wave radiation widths (Woosley et al. 1978; Rauscher \& Thielemann 2000). Past global systematics for S-wave radiation widths also suggest that the normalization may depend not only on mass number $A$, but also on average $\mathrm{S}$-wave resonance spacing's $\left(D_{0}\right)$ (Gardner 1975). We have investigated such a normalization. Rather than completely abandon our locally developed systematics for radiation widths, we consider a second normalization constant obtained by observing systematic behaviors in the ratio of recommended to calculated $30 \mathrm{keV}$ Maxwellian-averaged neutron capture cross sections as a function of $A$ and $D_{0}$, 

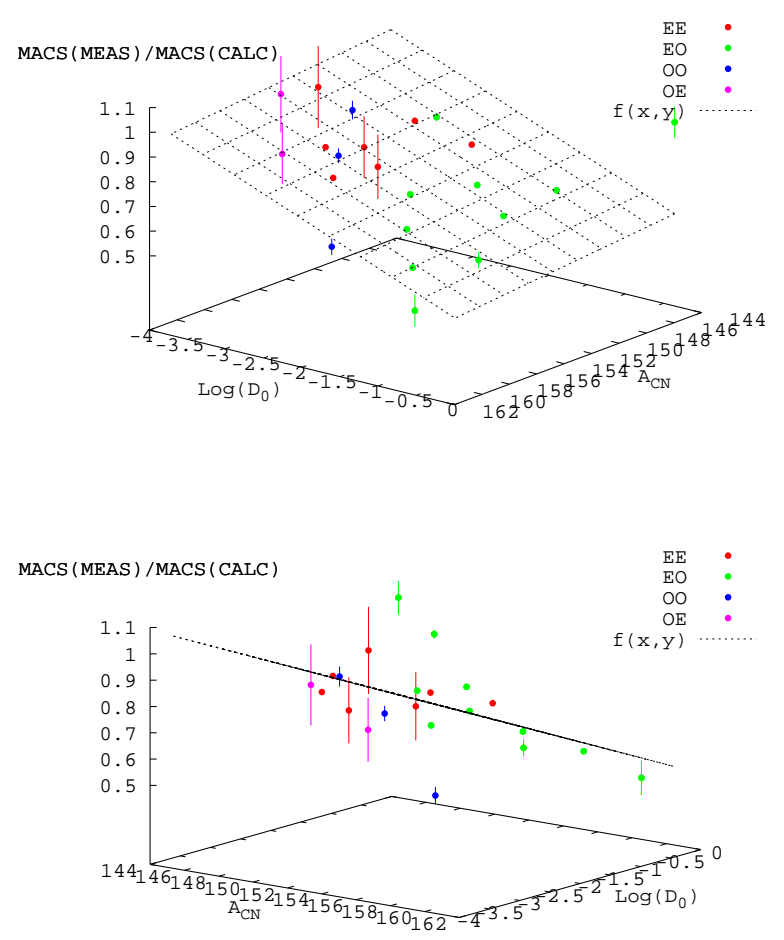

Fig. 10. - Systematics for a secondary normalization of the photon transmission coefficient based on replicating the $30-\mathrm{keV}$ Maxwellian-averaged neutron capture cross sections

see Figure 10.

The error bars on the points reflect errors in the measured Maxwellian-averaged cross sections (MACS). Red points represent even- $Z$ even- $N$ compound nuclei (resulting from neutron capture on even- $Z$ odd- $N$ targets), green points correspond to even- $Z$ odd- $N$ compound nuclei, blue points to odd- $Z$ odd- $N$ compound nuclei, and purple points to odd- $Z$ even- $N$ compound nuclei. The points are fairly well fit by a plane, with the exception of ${ }^{144} \mathrm{Sm},{ }^{155} \mathrm{Eu}$, and ${ }^{152} \mathrm{Gd}$ targets. We subsequently apply the ratio predicted by the systematic as a secondary normalization to the photon transmission coefficient.

To quantify the effect of this secondary normalization, we define a figure of merit

$$
g^{2}=\sum_{i=1}^{N}\left(\frac{x_{i}-y_{i}}{\sigma_{i}}\right)^{2}
$$

where $x_{i}$ are the recommended values, $\sigma_{i}$ are the corresponding errors in the recommended values, and $y_{i}$ are our calculated values. The sum extends over all $30 \mathrm{keV}$ MACS provided for samarium, europium, and gadolinium targets in (Bao et al. 2000). This figure of merit is similar to a weighted standard deviation, and thus a smaller number indicates a better replication of the recommended values. When only the radiation width systematic is employed, the figure of merit is 26.55 . When the secondary normalization is included, the figure or merit becomes 10.84 . Initially, this would seem to indicate that the inclusion of this secondary normalization would be an improvement. However, we note that the systematic in Figure 10 is a weighted fit, and is driven by the Maxwellian cross sections with the smallest error bars. Furthermore, any of the points in Figure 10 that lie between the systematic plane and the plane at unity will be brought into greater disagreement with the recommended values. These points include the compound nuclei formed in the critical neutron capture reactions on ${ }^{151} \mathrm{Eu}$ and ${ }^{152,153} \mathrm{Gd}$. Last of all, the application of this secondary normalization degrades the high degree of agreement between our calculations and measured neutron capture cross sections from (CSISRS 2003). As may be seen in Figure 21, our calculation (which does not include this secondary normalization) is in generally good agreement with the measured data, with the exception that our results for the ${ }^{156,158,160} \mathrm{Gd}(\mathrm{n}, \gamma)$, and to a lesser extent ${ }^{148,154} \mathrm{Sm}(\mathrm{n}, \gamma)$, cross sections are high. Our results for these five reactions improves greatly with the inclusion of the secondary normalization. However, our calculated cross sections for the more important ${ }^{151,153} \mathrm{Eu}(\mathrm{n}, \gamma)$ and ${ }^{152} \mathrm{Gd}(\mathrm{n}, \gamma)$ reactions, as well as ${ }^{155,157} \mathrm{Gd}(\mathrm{n}, \gamma)$, ${ }^{144,149} \mathrm{Sm}(\mathrm{n}, \gamma)$, and to a lesser extent ${ }^{152} \mathrm{Sm}(\mathrm{n}, \gamma)$ end up significantly lower than the measured data. Since the RADCHEM capture reactions of interest are largely on odd-N targets WE DO NOT ADOPT THIS SECONDARY NORMALIZATION to $30 \mathrm{keV}$ Maxwellian-averaged cross sections in our modeling effort.

\subsubsection{Comparison to experimental ( $n, 2 n)$ cross sections}

The ${ }^{151} \mathrm{Eu}(\mathrm{n}, 2 \mathrm{n}){ }^{150} \mathrm{Eu}$ cross section in Figure 9 shows good agreement with numerous experimental efforts. The data generally lies within three distinct regions for the activation, ground state, and first isomer cross sections. Our calculation fits these regions quite well, with the only notable exception being the isomer cross section around 10 $\mathrm{MeV}$. Consequently, the activation cross section is 
Table $6:{ }^{150} \mathrm{Eu}$ nuclear structure assignments.

\begin{tabular}{lcccc}
\hline \hline \multicolumn{3}{c}{ ground state } & \multicolumn{2}{c}{ isomer } \\
\hline year & $J^{\pi}$ & $\mathrm{T}_{1 / 2}$ & $J^{\pi}$ & $\mathrm{T}_{1 / 2}$ \\
\hline 1964 & $(4)$ & $\sim 5 \mathrm{y}$ & 1,0 & $12.8 \mathrm{~h}$ \\
1976 & $0^{(-)}$ & $12.62 \mathrm{~h}$ & $?$ & M1? few y \\
"” & & & $\left(4^{-}, 5^{-}\right)$ & M2 34.2 y \\
1986 & $5^{(-)}$ & $35.8 \mathrm{y}$ & $0^{(-)}$ & $12.8 \mathrm{~h}$ \\
1995 & $5^{(-)}$ & $36.9 \mathrm{y}$ & $0^{-}$ & $12.8 \mathrm{~h}$ \\
\hline
\end{tabular}

also slightly high around $10 \mathrm{MeV}$, but the differences are within $\sim 20 \%$. Predicting $(\mathrm{n}, 2 \mathrm{n})$ cross sections is fairly straightforward, as they scale roughly with the size of the nucleus, the activation cross section is typically about one and a half to two barns at $14 \mathrm{MeV}$ regardless of atomic number in this region of the nuclear chart.

\section{Uncertainty in the ${ }^{150}$ Eu ground state vs isomer assignment}

It is interesting to note the apparent confusion in the identification of the ground and isomeric state of ${ }^{150} \mathrm{Eu}$, as indicated by the multiple colored symbols (gray $=$ activation, orange $=$ ground state, blue=isomer) clustered around $14 \mathrm{MeV}$ in Figure 9 for ${ }^{151} \mathrm{Eu}(\mathrm{n}, 2 \mathrm{n}){ }^{150} \mathrm{Eu}$. The available experimental cross section data span a range of time from 1960 (Wille et al.) to 1999 (Filatenkov et al.), during which four evaluations of the basic nuclear structure data for the $A=150$ mass chain were made. Table 6 shows the spin, parity, and half-life assignments for the ground and isomer states of ${ }^{150} \mathrm{Eu}$ for each year.

In 1964 it was known that isomers existed in the $\mathrm{A}=150$ odd- $\mathrm{Z}$ isotopes, but their assignments were quite uncertain (Data Sheets 1964). The spin assignment to the long lived state in ${ }^{150} \mathrm{Eu}$ was a tentative one based on decay to $4^{+}$and $3^{-}$states in ${ }^{150} \mathrm{Sm}$ and the absence of decay to $2^{+}$levels. The $12.8 \mathrm{~h}$ lifetime of the shorter lived state was an adopted value from several measurements that ranged from 12.5 to $15 \mathrm{~h}$.

Little had changed by the time of the next evaluation (Nuclear Data Sheets 1976). Only one excited state (in $\mathrm{Tb}$ ) had been identified in the odd- $\mathrm{Z}$ isotopes of the $A=150$ chain, although the presence of isomers was still acknowledged. However, the excitation energy for each of these was unknown, so the ground state assignment was still unclear. Most of the authors who contributed to the evaluation assumed the low-spin $\mathrm{Eu}$ and $\mathrm{Tb}$ states should be associated with the ground state, subsequently the identity of the long and short lived states were switched. A plea for further measurements was made with the admission that shellmodel systematics were unreliable in this mass region and $\left(\beta^{+}+\epsilon\right)$ - decay did not generate definite assignments.

The two most recent evaluations agree on the assignment of the longer lived state being the ground state, and the uncertainties on the spin and parity of the isomer state have been removed (Nuclear Data Sheets 1986; Nuclear Data Sheets 1995). The $\sim$ few year state has been discarded from the level scheme. Even today, the ground state/isomer distinction is in fact still based on an unpublished IKP Report (Soromel-Stanco et al. 1982). In the LLNL radiochemistry detector sets Eu0988 and Eu0289, the ground state is associated with the $12.8 \mathrm{hr}$ isomer.

Analyzing the results of our ${ }^{153} \mathrm{Eu}(\mathrm{n}, 2 \mathrm{n}){ }^{152} \mathrm{Eu}$ calculation is slightly more involved, as there are many instances where the experiments do not agree with each other. Only two data sets are (definitely) activation cross sections. Our calculation is nearly within the error bar of the older set, and the newer set lies in the region of the ground state cross section data sets. The data sets with light gray error bars generally did not specify the state of the product nucleus. Our calculation for the ground state cross section is only slightly lower than the data, by roughly $15 \%$. Our calculation shows a marked difference with respect to the isomer cross sections. In that the isomer cross sections are in good agreement with several experiments (the obvious exception being the second isomer cross section of 2 barns at $14.7 \mathrm{MeV}$ ), we tend to believe that the data is correct. It is somewhat comforting to note that the sum of the two isomer cross section is nearly the same for both our calculation and the experimental data, differing by $\approx 30 \%$ at most. The likely cause of errors in the amount of cross section going to each isomer lies within our adopted set of discrete levels for ${ }^{152} \mathrm{Eu}$. In particular, some of the key branching ratios we have adopted are uncertain. It is possible, for instance that several excited states above the second isomer are decaying to the second isomer in our adopted level scheme, when they could in reality be decaying to the first isomer. One might try to alleviate these errors by simply including fewer levels. However, in our analysis the activation to continuum states is assumed to distribute itself to the ground state and isomers, weighted by a factor of $(2 J+1) / \sum_{i}\left(2 J_{i}+1\right)$ where $J$ is the spin of 
the state in question and the sum is over all possible final states. The spins of the ground state and first isomer of ${ }^{152} \mathrm{Eu}$ are, respectively, $J_{0}=3$ and $J_{1}=0$. The second isomer is a high-spin isomer of $J_{2}=8$. Hence, in the weighted distribution of the continuum population, the majority would end up in the second isomer. As this would result in a higher second isomer cross section, this would only enhance the disagreement with the data. Without more detailed spectroscopic data, it is not likely that this problem can be resolved.

Our calculations for several additional experimental $(\mathrm{n}, 2 \mathrm{n})$ cross sections on Sm and Gd targets are presented in Figure 23. These exhibit overall reasonable agreement (within $\sim 20 \%$ ), similar to the ${ }^{151,153} \mathrm{Eu}(\mathrm{n}, 2 \mathrm{n})$ activation cross sections. There are some noticeable deviations where our calculation tends to be higher than the data on the rising edge of the cross section. In all of these cases the experimental data is provided by a single author (Frehault 1975).

We again present in Table 7 a quantified comparison of our results to the experimental data shown in Figures 9 and 23. We include all data with incident energies of $14.7 \pm 0.1 \mathrm{MeV}$. The format of the table is identical to Table 5 .

The average deviation of our cross sections from the weighted means for the $(n, 2 n)$ reactions is roughly $10 \%$ when only activation measurements are considered. The largest deviations occur for the cross sections to specific final states (ground state, isomers). When these are included in the average, the deviation is closer to $35 \%$. This is due largely to the ${ }^{153} \mathrm{Eu}$ reaction going to the second isomer, where the absolute error between our calculation and the measured data is on the order of $400 \%$.

\subsubsection{Comparison to experimental $(p, n)$ cross sections}

The ${ }^{151} \mathrm{Eu}(\mathrm{p}, \mathrm{n}){ }^{151} \mathrm{Gd}$ cross section in Figure 9 is in agreement with (Nethaway \& Mustafa 1999) up to about $7 \mathrm{MeV}$. Our calculation peaks at the same energy as the data, although it is high by $\sim 10 \%$. Beyond the peak between $\sim 11-14 \mathrm{MeV}$, our calculation again agrees well with the data. Above $14 \mathrm{MeV}$, our calculation runs high.

The behavior of the ${ }^{153} \mathrm{Eu}(\mathrm{p}, \mathrm{n}){ }^{153} \mathrm{Gd}$ cross section is similar to that of the ${ }^{151} \mathrm{Eu}(\mathrm{p}, \mathrm{n}){ }^{151} \mathrm{Gd}$ case, except that we underestimate the peak by $\sim 10 \%$ rather than overestimate. Above $14 \mathrm{MeV}$ our calculation remains high.

There are many possible causes of this over es-
Table 7: Comparison of our modeled $(\mathrm{n}, 2 \mathrm{n})$ cross sections to experimental data at $14.7 \pm 0.1 \mathrm{MeV}$

\begin{tabular}{cllllll}
\hline \hline${ }^{A} Z$ & Res. & $\mathrm{N}$ & $\bar{\sigma}$ & Dev. & $\sigma_{\text {mod. }}$ & $\bar{\sigma} / \sigma_{\text {mod }}$ \\
\hline${ }^{144} \mathrm{Sm}$ & GS & 10 & 0.941 & 0.223 & 0.676 & 1.392 \\
${ }^{144} \mathrm{Sm}$ & M1 & 17 & 0.561 & 0.083 & 0.773 & 0.726 \\
${ }^{144} \mathrm{Sm}$ & A & 14 & 1.423 & 0.219 & 1.449 & 0.982 \\
${ }^{148} \mathrm{Sm}$ & A & 1 & 1.783 & & 1.852 & 0.963 \\
${ }^{150} \mathrm{Sm}$ & A & 1 & 1.774 & 0.207 & 1.906 & 0.931 \\
${ }^{152} \mathrm{Sm}$ & A & 1 & 1.840 & & 1.934 & 0.952 \\
${ }^{154} \mathrm{Sm}$ & A & 7 & 1.771 & 0.447 & 1.988 & 0.891 \\
${ }^{151} \mathrm{Eu}$ & GS & 8 & 1.042 & 0.330 & 1.229 & 0.848 \\
${ }^{151} \mathrm{Eu}$ & M1 & 9 & 0.705 & 0.361 & 0.591 & 1.195 \\
${ }^{151} \mathrm{Eu}$ & A & 4 & 1.338 & 0.310 & 1.819 & 0.736 \\
${ }^{153} \mathrm{Eu}$ & GS & 2 & 1.549 & 0.009 & 1.244 & 1.245 \\
${ }^{153} \mathrm{Eu}$ & M1 & 6 & 0.317 & 0.076 & 0.180 & 1.767 \\
${ }^{153} \mathrm{Eu}$ & M2 & 4 & 0.074 & 0.011 & 0.368 & 0.201 \\
${ }^{153} \mathrm{Eu}$ & A & 3 & 1.641 & 0.250 & 1.791 & 0.916 \\
${ }^{152} \mathrm{Gd}$ & A & 1 & 1.867 & & 1.849 & 1.010 \\
${ }^{154} \mathrm{Gd}$ & A & 3 & 1.582 & 0.559 & 1.892 & 0.836 \\
${ }^{155} \mathrm{Gd}$ & A & 1 & 1.843 & & 1.970 & 0.936 \\
${ }^{156} \mathrm{Gd}$ & A & 1 & 1.730 & & 1.955 & 0.885 \\
${ }^{157} \mathrm{Gd}$ & A & 1 & 1.820 & & 2.019 & 0.901 \\
${ }^{158} \mathrm{Gd}$ & A & 1 & 1.794 & & 2.020 & 0.888 \\
${ }^{160} \mathrm{Gd}$ & A & 9 & 1.863 & 0.439 & 2.013 & 0.926 \\
\hline & & & \multicolumn{3}{c}{ Average error: } & $33.5 \%$ \\
\hline & Average error (activation & only): & $10.2 \%$ \\
\hline
\end{tabular}

timation. One possibility is our use of a simple exciton pre-equilibrium model. In Figure 11 we compare the pre-equilibrium fraction (defined as the fraction of the absorption cross section resulting in pre-equilibrium reactions) from our model against a more sophisticated Monte Carlo treatment found in the ALICE code. In this figure, the solid red line represents the results from ALICE, and the dotted blue line represents our result. Reactions involving ${ }^{151} \mathrm{Eu}$ and ${ }^{153} \mathrm{Eu}$ are found on the top two panels and bottom two panels, respectively. The exciton model calculations only extend down to the threshold of the respective $(n, 2 n)$ and $(\mathrm{p}, \mathrm{n})$ reactions.

It is apparent from Figure 11 that the exciton model has a tendency to overestimate the preequilibrium fraction in comparison to the more sophisticated model. In the case of the two $(n, 2 n)$ reactions, the over estimation is only on the order of $20-25 \%$ at $14 \mathrm{MeV}$, which would result in a similar increase in the pre-equilibrium cross section. Pre-equilibrium emission is only considered for the first compound nucleus, and hence reactions involving only a single particle in the exit 




Fig. 11. - Comparison of pre-equilibrium fractions resulting from the ALICE Monte Carlo model and the simple exciton model in STAPRE.

channel will be enhanced. The enhancement of these channels result in a smaller remaining portion of the absorption cross section, and consequently the $(\mathrm{n}, 2 \mathrm{n})$ cross sections are reduced by the inclusion of pre-equilibrium. For these two particular $(\mathrm{n}, 2 \mathrm{n})$ reactions, pre-equilibrium emission lowers the total cross section at $14 \mathrm{MeV}$ by $\sim 23 \%$, and substitution of the Monte Carlo preequilibrium fraction would only result in a $\sim 5 \%$ increase in the total cross section. Below $14 \mathrm{MeV}$, pre-equilibrium contributions become less important, and the total cross section becomes less sensitive to the pre-equilibrium model. Above $14 \mathrm{MeV}$, we do not have measured $(\mathrm{n}, 2 \mathrm{n})$ data to compare against.

The case is quite different for the $(\mathrm{p}, \mathrm{n})$ reactions. At $10 \mathrm{MeV}$, the approximate location of the two $(\mathrm{p}, \mathrm{n})$ peaks, pre-equilibrium emission only accounts for $\sim 5 \%$ of the total cross section. By 14 $\mathrm{MeV}$, this fraction has grown to $\sim 55 \%$, and at $20 \mathrm{MeV}$ it is $\sim 97 \%$. At $14 \mathrm{MeV}$, the over estimation of the pre-equilibrium fraction is roughly $50 \%$ (see Figure 11), and using the Monte Carlo model would likely result in a $\sim 25 \%$ reduction in the total $(\mathrm{p}, \mathrm{n})$ cross section. This would improve the agreement between our calculation and the measured data for ${ }^{151} \mathrm{Eu}(\mathrm{p}, \mathrm{n}){ }^{151} \mathrm{Gd}$ at $14 \mathrm{MeV}$. For ${ }^{153} \mathrm{Eu}(\mathrm{p}, \mathrm{n})^{153} \mathrm{Gd}$ the calculation would end up low at $14 \mathrm{MeV}$. At $20 \mathrm{MeV}$, the over estimation of the pre-equilibrium fraction is on the order of $40 \%$. Since pre-equilibrium emission accounts for nearly all of the cross section at $20 \mathrm{MeV}$, using the Monte Carlo pre-equilibrium model would lower the (p,n) cross sections by roughly $40 \%$, bringing both calculations into much better agreement with the measured data.

At the present time, Monte Carlo pre-equilibrium has not been integrated into the STAPRE code. In the UGT analysis, our calculations for charged particle reactions on the stable europium targets will be replaced (Nethaway \& Mustafa 1999). The $(\mathrm{p}, \mathrm{n})$ and $(\mathrm{d}, 2 \mathrm{n})$ reactions are the largest cross sections affecting the production of ${ }^{151,153} \mathrm{Gd}$.

\subsubsection{Comparison to experimental (d,2n) cross sections}

The shapes of the calculated $(\mathrm{d}, 2 \mathrm{n})$ cross sections for ${ }^{151,153} \mathrm{Eu}$ in Figure 9 agrees well with the shape of the measured cross sections. Our calculation for the ${ }^{151} \mathrm{Eu}(\mathrm{d}, 2 \mathrm{n})$ peak is high by $22 \%$. The agreement on the rise from threshold and in the high energy tail is better. For the ${ }^{153} \mathrm{Eu}(\mathrm{d}, 2 \mathrm{n})$ cross section, we underestimate the peak by $\sim 10 \%$. While difficulties in replicating these cross sections may lie partially in the level densities and pre-equilibrium treatment, we also note that the deuteron optical potential utilized is a global potential that assumes spherical nuclei, as opposed to the neutron and proton potentials which involve coupled channels to ac- 
count for collective motion. We will investigate the role played by the deuteron potential further in the next section. Again, we emphasize that the ${ }^{151,153} \mathrm{Eu}(\mathrm{d}, 2 \mathrm{n})$ cross sections used in UGT analysis will be those of (Nethaway \& Mustafa 1999). However, our modeled $(\mathrm{p}, \mathrm{n})$ and $(\mathrm{d}, 2 \mathrm{n})$ reactions will be used should Sm be considered as a loaded detector element.

\subsubsection{Comparison to other experimental (n,x) cross sections}

Finally, Figures 24-26 in Appendix B show the available $(n, 3 n),(n, p),(n, \alpha)$, and $(n, d)$ cross sections. The experimental data is often sparse, and not infrequently exhibits large error bars in gross disagreement with other experiments (or our modeled results) which brings into question the reliability of cross sections from such authors. It is also often available only over a very limited energy range. In nearly all instances our calculations are in good agreement. We note that the $(n, p),(n, \alpha)$ and $(\mathrm{n}, \mathrm{d})$ cross sections in this region are all small, $\sim 25 \mathrm{mb}$ for $(\mathrm{n}, \mathrm{p}), \sim 10 \mathrm{mb}$ for $(\mathrm{n}, \alpha)$, and $\sim 1 \mathrm{mb}$ for $(n, d)$, when compared to the larger $(n, \gamma)$ and $(\mathrm{n}, 2 \mathrm{n})$ neutron induced cross sections. The reason is obvious, the Coulomb barrier in the exit channel prohibits charged particle emission, just as it does in absorption in the entrance channel. Such cross sections should not have a significant impact on RADCHEM analysis and have often been ignored in previous treatments. Our calculations seem to justify this.

\subsection{Sensitivity Studies}

We now illustrate the sensitivity of our modeled results to variations in the input parameters developed in $\S 3$ against the measured activation cross sections for $(n, \gamma),(n, 2 n),(p, n)$, and $(\mathrm{d}, 2 \mathrm{n})$ reactions on ${ }^{151} \mathrm{Eu}$ and ${ }^{153} \mathrm{Eu}$ targets.

\subsubsection{Sensitivity to the Pre-Equilibrium Cross Section}

We adopt a simple exciton model with initial 2-particle 1-hole configuration. Average rates for internal transitions are related by the formulas of Williams (1970), corrected for the Pauli principle by Cline (1972), to the absolute square of the average effective matrix element $|M|$ of the residual interactions as per Eq. [7] of (Uhl \& Strohmaier 1976). The dependence of $|M|^{2}$ on mass number and excitation energy is

$$
|M|^{2}=\langle F M\rangle A^{-3} E^{-1}
$$

Figure 12 shows the sensitivity of the $(n, 2 n)$ and $(\mathrm{p}, \mathrm{n})$ cross sections of ${ }^{151,153} \mathrm{Eu}$ targets for variations of $100 \leq\langle F M\rangle \leq 400$. The variations affect the high energy tail of the $(\mathrm{p}, \mathrm{n})$ cross section, with a value of $\langle F M\rangle=400$ providing the best agreement with the experimental excitation function. However, it is difficult to justify on any physical basis such a high value for $\langle F M\rangle$. Additionally, using $\langle F M\rangle=400$ tends to result in poor agreement with measured data for reactions with charged particles in the exit channel. For these reactions, $\langle F M\rangle=300$ is preferred. The $(\mathrm{p}, \mathrm{n})$ cross sections differ significantly for $\langle F M\rangle=300$ and $\langle F M\rangle=400$ only above $14 \mathrm{MeV}$. As noted previously, the over estimation of the $(\mathrm{p}, \mathrm{n})$ cross sections at incident energies above $14 \mathrm{MeV}$ is likely due to the simplicity of the exciton model. Hence, we adopt a value of $\langle F M\rangle=300$ for all reaction with charged particles in the incident or outgoing channels.

For the reasons stated in Section 4.1, accurately modeling the charged particle reactions on the loaded europium isotopes is not as critical as accurately modeling the neutron cross sections. We note that $\langle F M\rangle=300$ tends to overestimate the $(\mathrm{n}, 2 \mathrm{n})$ cross sections, with $\langle F M\rangle=200$ providing a better match to the data in Figure 12, as well as numerous other $(\mathrm{n}, 2 \mathrm{n})$ and $(\mathrm{n}, 3 \mathrm{n})$ cross sections in the local region of interest where experimental data was available. Hence, for reactions with neutrons only in the incident and exit channels, we adopt $\langle F M\rangle=200$.

\subsubsection{Sensitivity to the Level Density - Various Prescriptions}

The nuclear level density parameters developed for this region (Table 12, Appendix A.5) reflect best choices from the available experimental data in the region of interest. In this section we provide comparisons against three previously developed level density prescriptions where only the overall treatment of the level density prescription was varied, we keep all other parameter input (transmission coefficients, pre-equilibrium, etc.) fixed. An investigation into the effect of varying only the shell correction $\left(\delta_{W}\right)$ in our prescription will be given in the next section.

Figure 13 shows the experimental cross sections for $(n, \gamma),(n, 2 n)$, and $(p, n)$ cross sections on ${ }^{151,153} \mathrm{Eu}$ (for the sources of the experimental data, (CSISRS 2003)). Only data for the activation cross section is shown. For comparison we show our local systematic level density (red-solid 

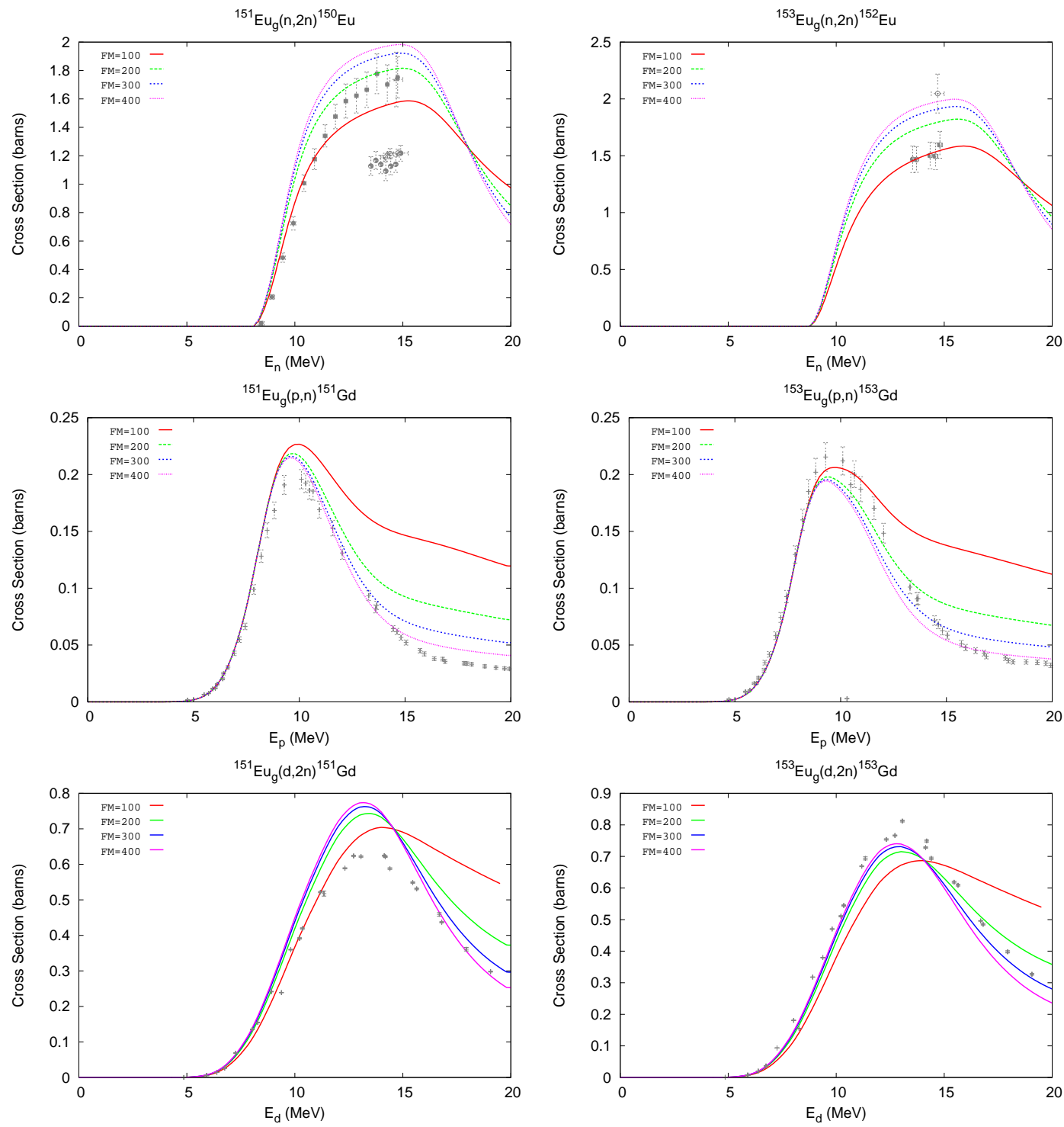

Fig. 12.- Sensitivity to pre-equilibrium matrix element FM.

line, Table 12).

The second level density prescription from (Rauscher et al. 1997) is plotted as the purple dashed line. This prescription is the current standard used in the calculation of cross sections for use in stellar nucleosynthesis calculations (Rauscher et al. 2002). The prescription is very similar to ours, in that our choice for the parameterization of the spin cutoff parameter, backshift, and asymptotic level density parameter are taken directly from this prescription. It differs from ours only in the choice of shell correction $\delta W$. Ours is derived from measured resonance spacing's or the associated local systematic, while the shell corrections for this prescription are the microscopic ground state energies from (Möller et al. 1995).

A third level density prescription is shown as the blue dotted line (Gilbert \& Cameron 1965). This is one of the original prescriptions available and has been used to model some of the cross sections in the current RADCHEM sets (Vonach 1982). This prescription includes pairing and shell corrections but with an energy independent $a(A)$ parameter. It also served as the basis of the prescription used in the first large scale theoretical cross section library developed for use in modeling pre-supernova nucleosynthesis in stars [(Woosley et al. 1978), (Woosley \& Weaver 1995)], and provided remarkable success in predicting measured solar abun- 

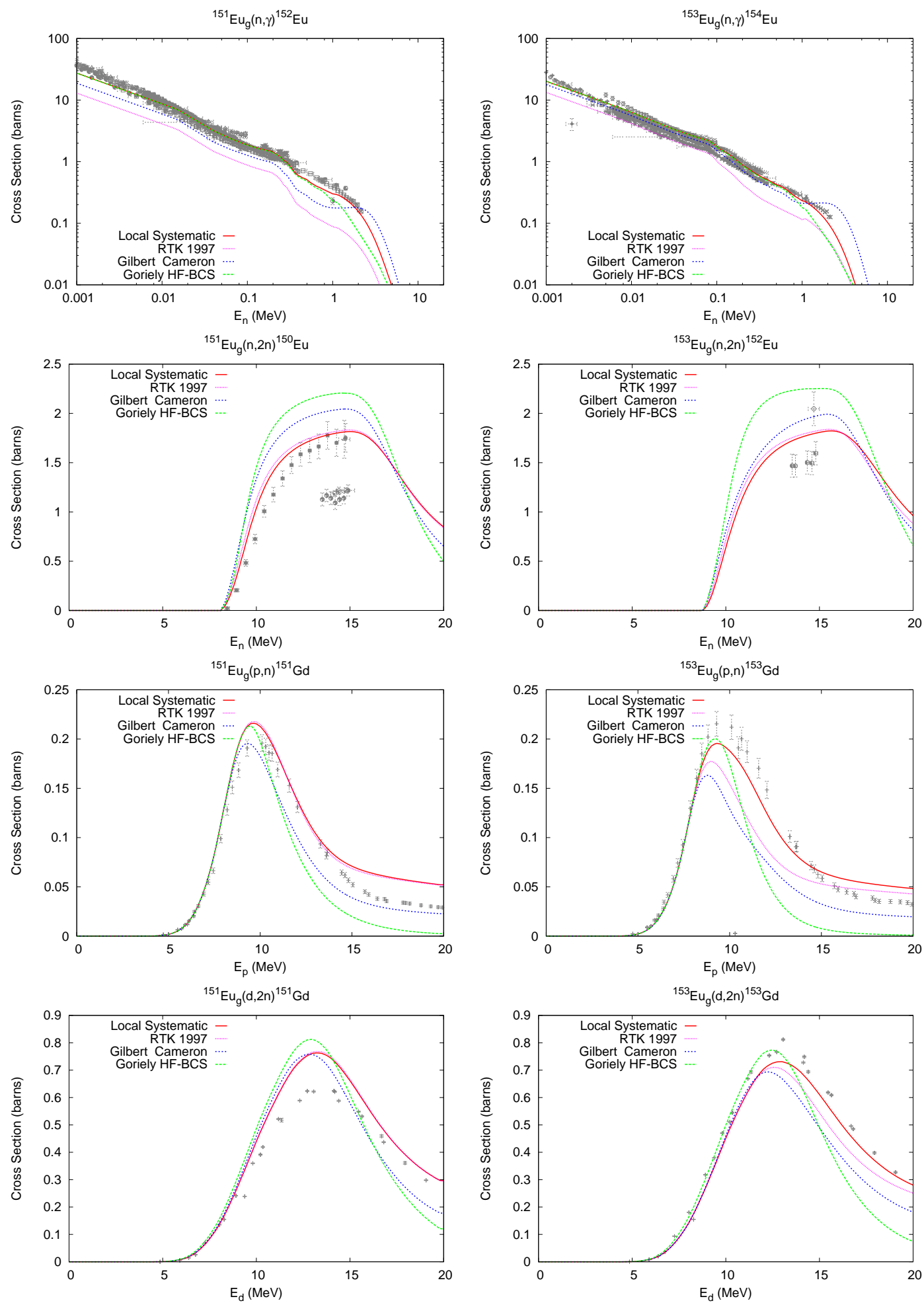

Fig. 13.- Sensitivity to the choice of level density prescription $\rho(U)$.

dances within the context of a study of Galactic Chemical Evolution (i.e. radchem for astrophysics). See (Timmes Woosley \& Weaver 1995).
The last choice (green dashed line) reflects a recent attempt to calculate level densities using microscopic nuclear structure models (Goriely 2002). 
For the $(\mathrm{n}, \gamma)$ capture reactions, we see that our prescription as well as that of Goriely predict the same cross section below $200 \mathrm{keV}$, and are in good agreement with the experimental data below 200 $\mathrm{keV}$. In the case of the ${ }^{153} \mathrm{Eu}$ target, the level densities of (Gilbert \& Cameron 1965) also perform well. The Gilbert and Cameron and Rauscher level densities tend to result in smaller cross sections. The overall behavior of the cross sections up to $1 \mathrm{MeV}$ is quite similar. Above $1 \mathrm{MeV}$, the Goriely and Gilbert and Cameron cross sections exhibit different behaviors. Of course above $1 \mathrm{MeV}$ the values of these cross sections begin to drop rapidly, and would not compete with particle induced reactions for these higher energies. Overall, we believe our local systematic is in good agreement for the important energy range between 10 and $100 \mathrm{keV}$.

For the $(n, 2 n)$ reaction we see that the three Fermi-Gas level densities all predict roughly the same cross section over the entire range of excitation energy studied. The Gilbert and Cameron level density results in a somewhat higher cross section in both cases, by roughly 5-15\%. Again, we consider our local systematic to be preferable to any of the other three. For the ${ }^{153} \mathrm{Eu}$ target, we suggest the data points clustered around 1.5 barn at $14 \mathrm{MeV}$ are misidentified by (CSISRS 2003), they likely reflect the cross section to the ground state of ${ }^{152} \mathrm{Eu}$.

The (p,n) cross sections exhibit significant sensitivity to the level density prescription with large variations in the size of the cross section at the peak, and the rate at which the cross section falls off after the peak. For ${ }^{151} \mathrm{Eu}(\mathrm{p}, \mathrm{n})$, our level density prescription and that of (Rauscher et al. 1997) result in very similar cross sections, and are superior to the other two prescriptions in replicating the cross section up to $14 \mathrm{MeV}$. Above $14 \mathrm{MeV}$, the prescription of (Gilbert \& Cameron 1965) appears superior, but we must reiterate that the over estimation of the cross section using our level densities is most likely due to the simple nature of the exciton model. For ${ }^{153} \mathrm{Eu}(\mathrm{p}, \mathrm{n})$, our level density is clearly superior to the other prescriptions below $14 \mathrm{MeV}$. The results for the $(\mathrm{d}, 2 \mathrm{n})$ reactions are similar to those of the $(\mathrm{p}, \mathrm{n})$ reactions. We again emphasize that the actual measured charged particle induced cross sections in (Nethaway \& Mustafa 1999) will be used in the UGT analysis.

\subsubsection{Sensitivity to the Level Density - Shell Correction Systematic}

The systematic presented in Figure 6 represents a least squares fit to shell corrections $(\delta W)$ derived from known resonance spacing's, assuming a specific form for other Fermi gas level density parameters ( $\S 3.3)$. The error bars correspond to errors in the measured resonance spacing's, obtained by deriving shell corrections from the upper and lower limits of the measurement. The average size of these error bars is $0.38 \mathrm{MeV}$, with $82 \%$ of them being smaller than $0.5 \mathrm{MeV}$.

In Figure 14 we present the effect of varying the shell corrections up or down by $0.5 \mathrm{MeV}$. In each case, the matching energy $E_{x}$ was refit to the spectroscopic data, so that the level density below $E_{c u t}$ (i.e. in the region of known spectroscopic levels) remains unchanged.

We note relatively small changes in the capture cross sections, adding $0.5 \mathrm{MeV}$ to $\delta W$ raised the cross sections by roughly $15 \%$, subtracting 0.5 $\mathrm{MeV}$ made little difference (non-intuitively, it increased it). Below $\sim 1 \mathrm{MeV}$, the changes will be due to corresponding adjustments in the normalization of the photon transmission function that arise from modifications to the level density.

The $(\mathrm{n}, 2 \mathrm{n})$ cross sections exhibit minimal sensitivity to variations in the shell correction. A somewhat more significant, yet still minor, sensitivity occurs in the charged particle induced reactions. The highest degree of sensitivity occurs in the ${ }^{153} \mathrm{Eu}(\mathrm{p}, \mathrm{n})$ cross section, where a $0.5 \mathrm{MeV}$ increase in the shell correction increases the cross section at the peak by $\sim 10 \%$, being most sensitive to the level density of the residual nucleus ${ }^{153} \mathrm{Gd}$. The ${ }^{153} \mathrm{Gd}$ shell correction is derived from a measured resonance spacing, and has a value of 3.579 $\mathrm{MeV}$, with lower and upper limits of 3.141 and 4.129 MeV (see Figure 7 and Table 12, Appendix A.5).

\subsubsection{Sensitivity of (d,2n) Cross Sections to the Deuteron Optical Potential}

The optical model adopted for neutrons and protons (§3.2.3) includes provisions for collective vibrational and rotational motions (see Figure 1). We did not develop such potentials for deuterons and alpha particles, but rather adopted global prescriptions that neglect collective effects. Here we investigate the sensitivity to this input parameter by varying the choice of deuteron potential, leaving all other input fixed to our preferred pre- 

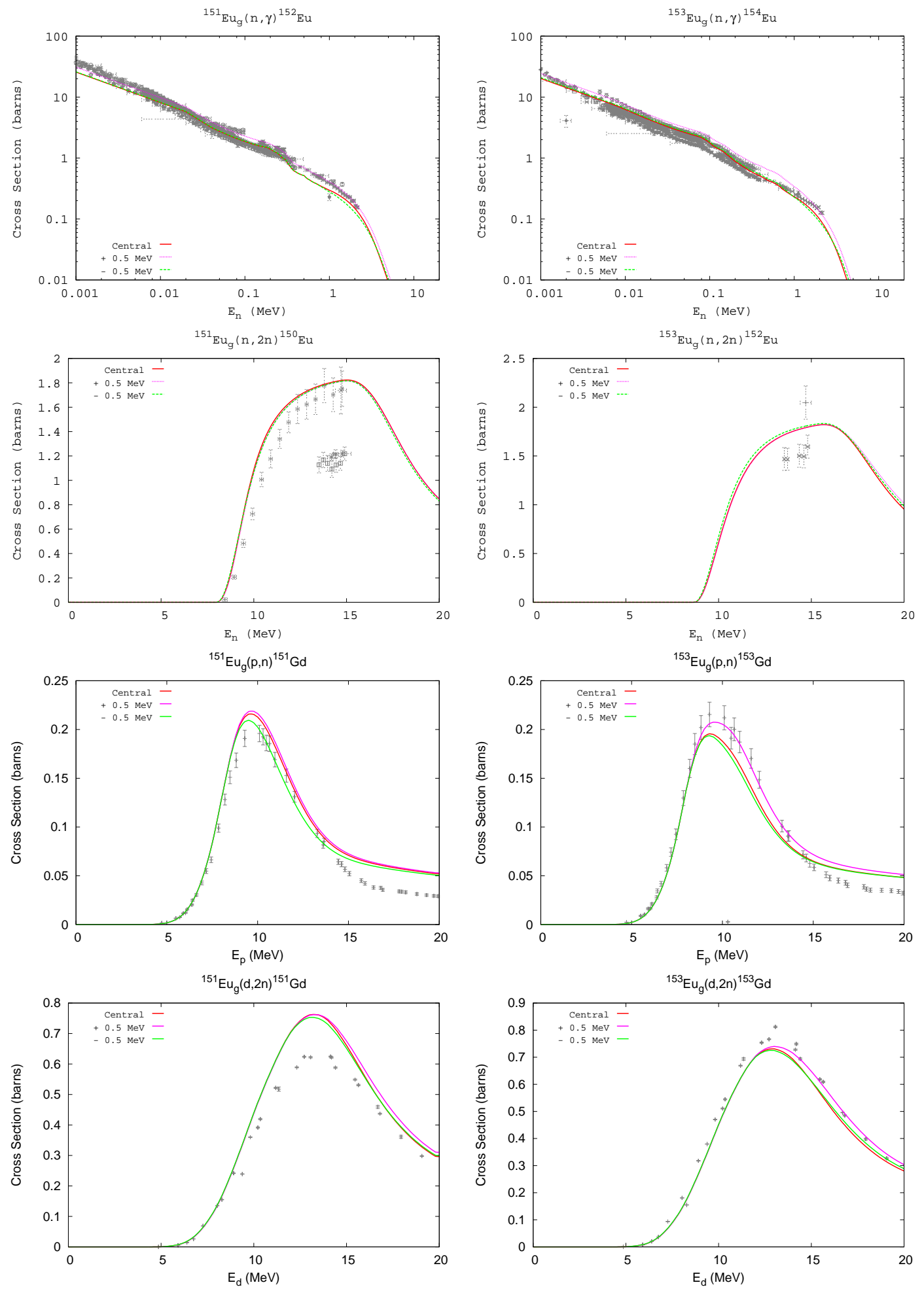

Fig. 14.- Sensitivity to variations in the level density shell correction $\delta W$.

scriptions developed in $\S 3$.

The upper two panels of Figure 15 show calculated $(\mathrm{d}, 2 \mathrm{n})$ cross sections for ${ }^{151,153}$ Eu targets us- ing the optical models of (Lohr \& Haeberli 1974) (solid red line) and (Perey \& Perey 1963) (dotted blue line). The measured cross section data is 
from (Nethaway \& Mustafa 1999). Interestingly, we obtain better agreement with experiment for the ${ }^{151} \mathrm{Eu}(\mathrm{d}, 2 \mathrm{n})$ cross section using the potential of (Perey \& Perey 1963), while the ${ }^{153} \mathrm{Eu}(\mathrm{d}, 2 \mathrm{n})$ cross section favors that of (Lohr \& Haeberli 1974). Ideally, a local optical potentials involving collective effects should be developed for deuterons and alpha particles. For the time being, we adopt the potential of (Lohr \& Haeberli 1974), on the basis that it includes spin-orbit terms.

Another sensitivity in calculating $(\mathrm{d}, 2 \mathrm{n})$ cross sections arises from the treatment of deuteron breakup in the presence of the Coulomb barrier (Udagawa \& Tamura 1986). Since STAPRE-H95 does not include such models, we operationally account for them by a suitable reduction of the deuteron total cross section (Mustafa 1997). For ${ }^{151} \mathrm{Eu}+\mathrm{d}$, we find that the total breakup cross section is about $40 \%$ of the optical model reaction cross section at $10 \mathrm{MeV}$ of incident deuteron energy, dropping to $\sim 31 \%$ around $20 \mathrm{MeV}$. Only about half of this enters through the $(\mathrm{d}, 2 \mathrm{n})$ exit channel, which translates to a roughly $20-30 \%$ reduction in the total deuteron reaction cross section. The breakup calculations do not vary greatly for $148 \leq A \leq 152$, so we include the suggested breakup calculated for ${ }^{151} \mathrm{Eu}+\mathrm{d}$ in our $(\mathrm{d}, 2 \mathrm{n})$ cross sections for all Eu and Sm targets.

The lower two panels in Figure 15 show the effects of inclusion (blue) and exclusion (red) of deuteron breakup on our ${ }^{151,153} \mathrm{Eu}(\mathrm{d}, 2 \mathrm{n})$ cross sections. Both used the optical model of (Lohr \& Haeberli 1974) (the calculations in the upper panels included deuteron breakup). The $(\mathrm{d}, 2 \mathrm{n})$ cross sections were overpredicted in both calculations if breakup was neglected.

\subsubsection{Sensitivity to the Normalization of the $\gamma$ - ray Transmission Coefficient}

Figure 16 shows the sensitivity of the stable $\mathrm{Eu}$ capture cross sections to a $\pm 30 \%$ change to the value of the average s-wave photon width used to normalize the gamma-ray transmission coefficients (§3.2.6, Figure 5). The $30 \%$ variation translates into a nearly identical change in the cross section from $10 \mathrm{keV}$ to $1 \mathrm{MeV}$. The $(\mathrm{n}, 2 \mathrm{n})$ and $(\mathrm{p}, \mathrm{n})$ cross sections do not exhibit any sensitivity because $\mathrm{T}_{\gamma}$ only enters into Eq. [1] in the denominator. In general, for capture reactions, the smaller of the two transmission coefficients in the HF numerator will be the one that determines the cross section, especially if it is much smaller. This is always the case with photon vs. particle widths. The mean
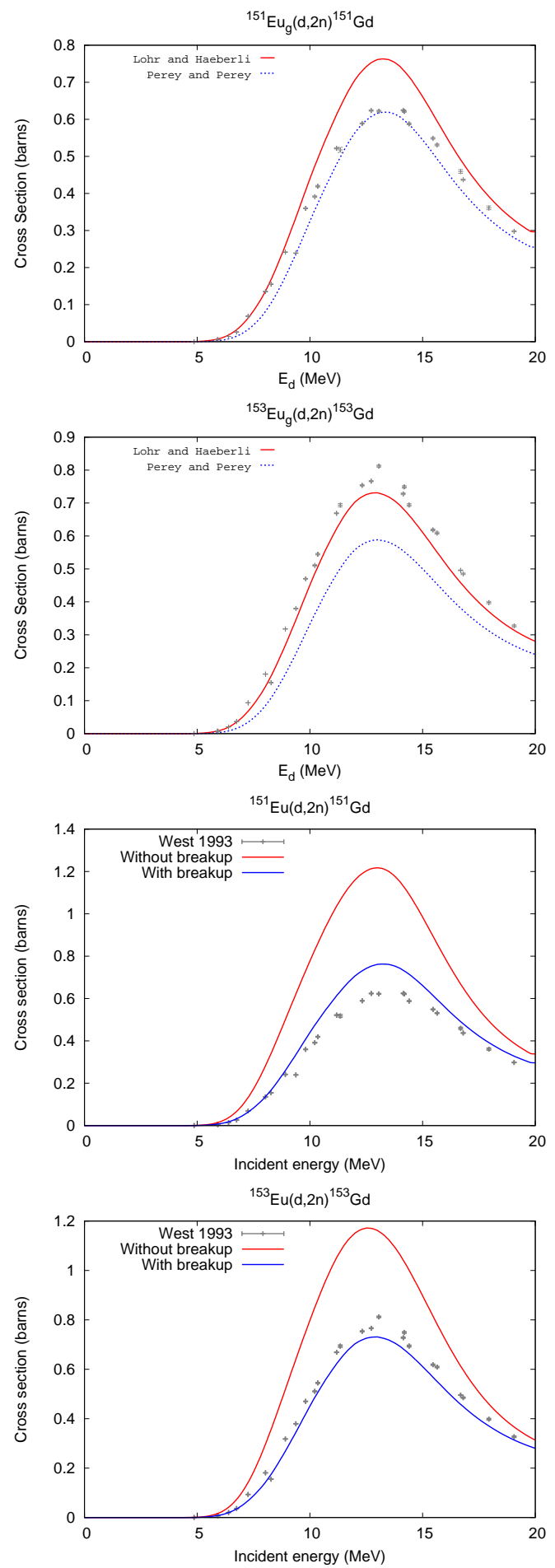

Fig. 15.- Sensitivity of $(\mathrm{d}, 2 \mathrm{n})$ cross sections to choice of deuteron optical potential (upper two panels) and the inclusion/exclusion of deuteron breakup (lower two panels). 



Fig. 16. - Sensitivity to a $\pm 30 \%$ adjustment of the experimental s-wave average photon width $\Gamma_{\gamma}$.

error associated with the experimental values of the gamma ray strength function for samarium, europium, and gadolinium isotopes is $12.6 \%$ with a standard deviation of $6 \%$. Hence, the uncertainties related to the gamma ray strength function in the capture cross sections is likely less than that shown in Figure 16.

\subsubsection{Sensitivity to the Inclusion of Width Fluc- tuation Corrections}

We adopt the Moldauer model of the WFC as embodied in the STAPRE code. For targets in the mass range of interest which have available experimental data, reaction thresholds are always greater than several $\mathrm{MeV}$, and width fluctuation corrections will only be evident for capture reactions. Figure 17 shows the affect for the activation capture cross sections of ${ }^{151,153} \mathrm{Eu}$ both with (solid red line) and without (green dashed line) WFC. As expected a decrease in the capture cross section is noticeable, in both cases less than $20 \%$ below $100 \mathrm{keV}$. When the projectile energy increases, the capture cross section declines rapidly and the elastic enhancement vanishes. The $(n, p)$ and $(p, n)$


Fig. 17.- Sensitivity to inclusion or exclusion of width fluctuation corrections $W$.

channels do not open until the incident neutron energy is well above $2 \mathrm{MeV}$, and so no other channels can compete with the elastic and capture cross sections before the WFC are negligible. Nearly identical behavior is noted for neutron capture on ${ }^{88} \mathrm{Sr}$ and ${ }^{93} \mathrm{Nb}$ (Hilaire Lagrange \& Koning 2003), see their Figures 2 and 3.

\subsubsection{Net Uncertainties in (n, $\gamma)$ Activation Cross Sections Due to Local Systematics}

Of the sensitivities presented thus far, the most significant for neutron capture cross sections are those that arise from estimated errors in our local systematics for the average total s-wave radiation width and the shell correction (or level density treatment in general).

In Figure 18 we present sensitivity of our calculated neutron capture cross sections that arise from simultaneous variations in these two systematics. This study was performed for the loaded stable targets and all the long-lived radioactivities in the RADCHEM Eu0289 and Eu0988 detector sets. The black lines represent our recommended cross section. The red lines indicate 


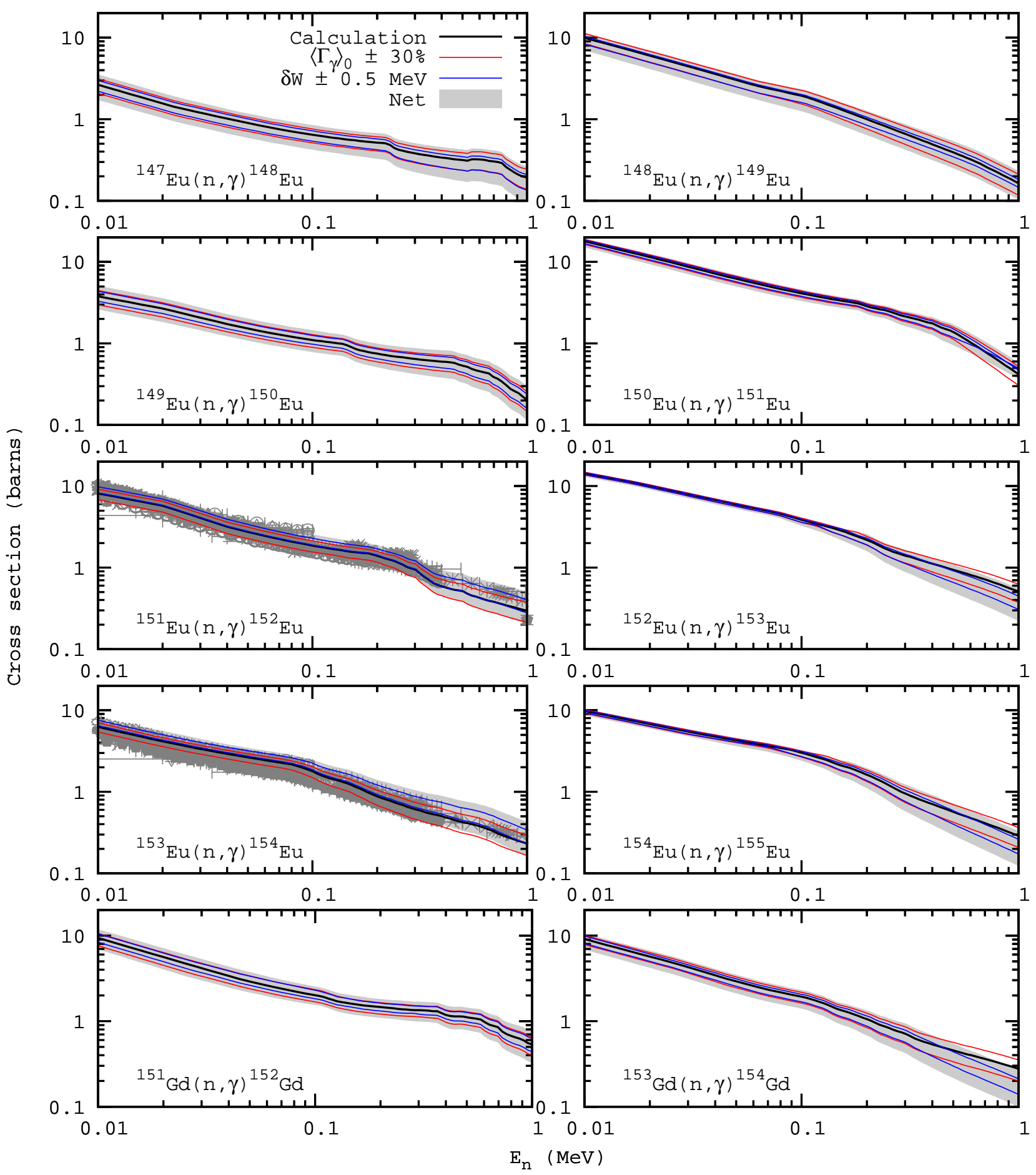

Fig. 18.- Uncertainties in select $30 \mathrm{keV}(\mathrm{n}, \gamma)$ activation cross sections due to simultaneous variations in $\Gamma_{\gamma}$ and $\delta W$.

the effect on the cross section when the radiation width (to which we normalize the photon transmission coefficient, Figure 5) is varied by $\pm 30 \%$. In all cases, an increase in the radiation width results in a larger cross section, a decrease in a lower cross section. The blue lines indicate the effect of altering the shell correction $\delta \mathrm{W}$ by \pm 0.5 $\mathrm{MeV}$. Generally, an increase in the shell correction yields an increase in the cross section, and vice versa. However, on occasion raising (lowering) the 
Table 8: Uncertainties in select $(\mathrm{n}, \gamma) 30 \mathrm{keV}$ activation cross sections arising from local systematics, expressed as an absolute percent difference from our recommended values

\begin{tabular}{lrrrrrr}
\hline \hline & \multicolumn{2}{c}{$\left\langle\Gamma_{\gamma}\right\rangle_{0}$} & \multicolumn{2}{c}{$\delta W$} & $(\mathrm{MeV})$ & \multicolumn{2}{c}{ Cumulative } \\
${ }^{A} Z$ & $+30 \%$ & $-30 \%$ & +0.5 & -0.5 & High & Low \\
\hline${ }^{147} \mathrm{Eu}$ & 18.0 & 21.1 & 12.8 & 16.5 & 32.8 & 34.1 \\
${ }^{148} \mathrm{Eu}$ & 13.3 & 17.0 & 3.5 & 17.8 & 16.9 & 32.4 \\
${ }^{149} \mathrm{Eu}$ & 17.6 & 20.5 & 14.7 & 12.8 & 33.5 & 30.3 \\
${ }^{150} \mathrm{Eu}$ & 6.8 & 10.5 & 4.7 & 7.9 & 10.9 & 19.2 \\
${ }^{151} \mathrm{Eu}$ & 13.6 & 17.1 & 22.4 & 1.2 & 36.7 & 17.1 \\
${ }^{152} \mathrm{Eu}$ & 3.1 & 5.1 & 1.5 & 4.7 & 4.3 & 10.7 \\
${ }^{153} \mathrm{Eu}$ & 10.6 & 13.8 & 20.3 & 3.5 & 33.4 & 13.8 \\
${ }^{154} \mathrm{Eu}$ & 4.8 & 7.3 & 2.9 & 6.1 & 7.3 & 12.2 \\
${ }^{151} \mathrm{Gd}$ & 13.9 & 19.6 & 12.4 & 11.5 & 28.0 & 28.2 \\
${ }^{153} \mathrm{Gd}$ & 11.8 & 14.8 & 6.4 & 12.4 & 18.3 & 27.1 \\
\hline Avg. & 11.4 & 14.7 & 10.1 & 9.4 & 22.2 & 22.5 \\
\hline
\end{tabular}

shell correction will lower (raise) the cross section. This behavior may arise from two factors. First, when a level density parameter is changed, the fit to low-lying spectroscopic levels is re-determined, again attempting to match the cumulative number of levels with the integrated level density at the highest energy up to which the level scheme is complete. Below this energy, the discrete levels are used. Second, any modification is the level density will be accompanied by a variation in the calculated radiation width with the overall normalization of the photon transmission coefficient set to unity. The factor $\left\langle\Gamma_{\gamma}\right\rangle_{0}^{\mathcal{N}=1} /\left\langle\Gamma_{\gamma}\right\rangle_{0}^{\text {Meas./Sys. }}$, and therefore the normalization of the photon transmission function, change with variations in the level density.

The gray hashed areas of Figure 18 indicate the range of uncertainty bounded by calculations using both larger values of $\left\langle\Gamma_{\gamma}\right\rangle_{0}$ and $\delta W$ or both smaller values. Again we note that in some instances lowering the shell correction results in a higher cross section.

We quantitatively evaluate these uncertainties in Table 8 which gives the absolute percent difference between the cross sections calculated using the variations in input depicted in Figure 18 and our recommended cross section at $E_{n}=30 \mathrm{keV}$. In this table the high and low cumulative uncertainties reflect the gray hashed areas in Figure 18, unless a calculation involving the variation of a single input parameter resulted in a larger difference. In such cases the larger percentage is listed.

The total uncertainties arising from these two parameters are on average about $22 \%$. This could account for at least half of the discrepancy between our calculated values and the measured cross sections listed in Table 5. This is not much larger than our demonstrated error in calculating the $(n, 2 n)$ cross sections $(10-15 \%)$.

\subsection{Production and Destruction Cross Sections}

Figure 19 shows the modeled cross sections that directly affect the production and destruction of ${ }^{151} \mathrm{Gd}$ and ${ }^{153} \mathrm{Gd}$. Identical plots arranged in order of increasing neutron number for all other targets listed in Table 9 are presented in Appendix C. In general, variations in a given cross section from target to target are small, although odd-even effects are apparent.

Of these the modeled $(\mathrm{p}, \mathrm{n})$ and $(\mathrm{d}, 2 \mathrm{n})$ cross sections are the largest charged-particle production cross sections. Secondary pathways exist that proceed through other isotopes, the plot suggest that the $(\mathrm{p}, 2 \mathrm{n})$ cross sections may also be of some importance in that they are considerably larger than $(\mathrm{p}, \mathrm{n})$ cross sections and have thresholds well below $14 \mathrm{MeV}$. But these cross sections proceed from unstable ${ }^{152,154} \mathrm{Eu}$, whose abundance must be build up by reactions on the stable Eu targets. By another route, $(\mathrm{p}, 2 \mathrm{n})$ reactions on stable $\mathrm{Eu}$ targets produce ${ }^{150,152} \mathrm{Gd}$, an additional $(\mathrm{n}, \gamma)$ reaction must take place to populate the radioactivity of interest to RADCHEM. Previously, $(\mathrm{p}, 2 \mathrm{n})$ cross sections were not included in the Eu0988 set.

Of the neutron induced destruction cross sections, the largest are the $(n, \gamma)$ capture cross sections (which become significant below $\sim 5 \mathrm{MeV}$ of excitation energy), and the $(n, 2 n)$ reactions. At intermediate energies between 4 and $7 \mathrm{MeV}$ (the $(\mathrm{n}, 2 \mathrm{n})$ threshold), $(\mathrm{p}, \mathrm{n})$ and $(\mathrm{d}, 2 \mathrm{n})$ become the dominant destruction channels. Charged particle destruction reactions on unstable Europium isotopes were not included in Eu0988 set. We do provide modeled charged-particle production reactions on all Sm targets, the stable ones populate the radioactive $\mathrm{Eu}$ isotopes of interest to RADCHEM.

Of course the particle fluences play a dominant role in determining the most important cross sections for RADCHEM. More specific details will be disclosed in a classified analysis presented in a subsequent paper. 

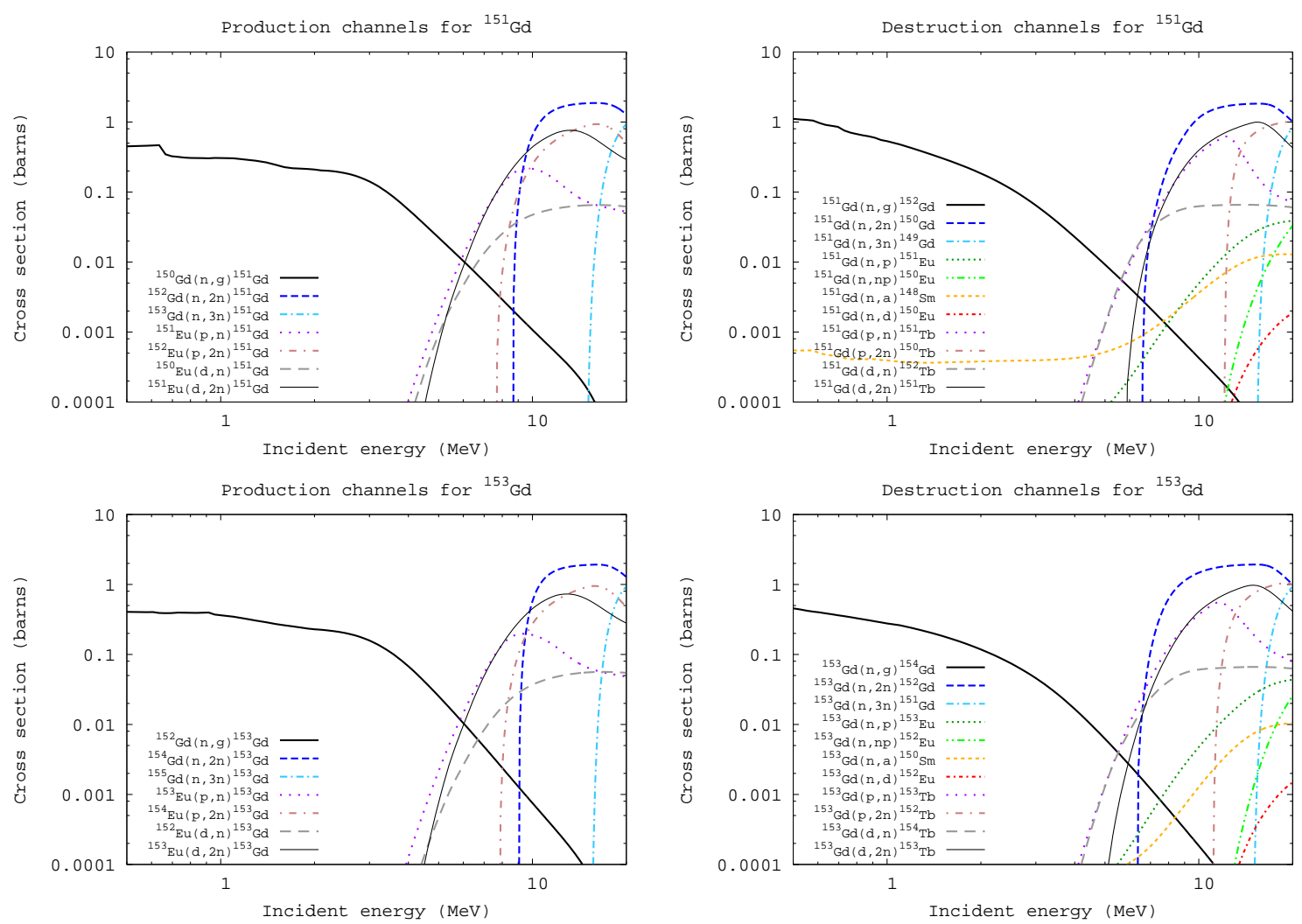

Fig. 19. - Calculated cross sections directly affecting production and destruction of ${ }^{151,153} \mathrm{Gd}$.

\section{Conclusions}

We have developed new neutron and charged particle induced cross section detector sets for radiochemical diagnostics of ${ }^{147-150,152,154} \mathrm{Eu}$ and

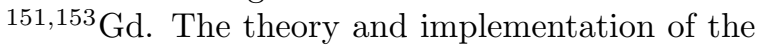
Hauser-Feshbach model was described ( $(2)$, along with the details of the local systematics used to create a set of input parameters that reflect the latest available experimental data in the local region of interest (§3). Modeled cross sections were compared to available experimental cross sections for the loaded detector elements (Figure 9), as well as other stable targets in the region (Appendix B). Sensitivity to reasonable variations in the input models and parameters was explored ( $\$ 4.2)$.

Overall we consider the modeling effort to be quite successful, as our calculated cross sections agree favorably with experimentally measured ones in this region of interest. In particular, we demonstrated an ability to calculate $(\mathrm{n}, 2 \mathrm{n})$ cross sections to about $10-15 \%$ accuracy (Table 7 ), and $(\mathrm{n}, \gamma)$ cross sections to within roughly $35 \%$ accuracy (Table 5). For charged-particle reactions we achieved an accuracy typically within $15-25 \%$.
We also provide suggested normalizations to our $(\mathrm{n}, 2 \mathrm{n})$ and $(\mathrm{n}, \gamma)$ capture cross sections on stable targets to bring them into agreement (in an average sense) with the body of experimental data that exists.

In our attempts to model neutron capture cross sections it should be kept in mind that we are considering compound nuclear systems for which the important input parameters to our reaction model (e.g. those that affect level densities and photontransmission coefficients) are often determined by normalization to experimental data (e.g. from resonance analysis), and so one would expect comparisons to measured capture cross sections to be good. Since these compound nuclei often bracket the systems of most interest to us, namely those which account for the dominant destruction reactions like $(\mathrm{n}, \gamma)$, our systematics should reasonably provide for similar agreement. Width fluctuation corrections are also an important ingredient.

For the $(\mathrm{n}, 2 \mathrm{n})$ and charged-particle induced reactions, the treatment of particle transmission coefficients (e.g. the optical model) and preequilibrium are most important. The former we treat with great care due to the deformed nature of 
this particular region. The later we employ a fairly straight forward model for, largely to normalize cross section behavior at and above $14 \mathrm{MeV}$.

\subsubsection{Recent LLNL experimental efforts}

This modeling effort has benefited from recent collaborative work between the Experimental Nuclear Physics (ENP) and Nuclear Theory and Modeling (NTM) groups in N-Division (PAT) through the development of the "surrogate reaction" technique, which is the subject of a current LDRD effort in N-Division (Escher et al. 2004). If shown to be successful, this technique could enable the determination of neutron induced cross sections on unstable targets.

In brief, the method attemtps to populate a particluar compound nucleus through a direct or transfer reaction, and then measure aspects of its decay products (either particles or photons). Coupled with nuclear theory, the experimental results are used to infer neutron-induced cross sections on unstable targets that would have populated the same compound nucleus through neutron capture. The technique rests on the theory of nuclear amnesia $(\S 2)$, once formed the compound nucleus "forgets" the way in which it was populated. We are testing this theory by examining if certain constants of the reaction are conserved (the angular momentum and parity distributions of incident neutrons vs. charged particles are different).

So far two experiments have been performed, one in the rare-earth region and one near the mass 90 closed shell region. Both were chosen to establish the validity of the method in the special case where the desired cross section had already been measured using traditional techniques.

The first experiment (Bernstein et al. 2005) studied $45 \mathrm{MeV}$ incident ${ }^{3} \mathrm{He}$ nuclei on a highly enrighed ${ }^{157} \mathrm{Gd}$ target. Compound nuclei of ${ }^{156} \mathrm{Gd}$ and ${ }^{157} \mathrm{Gd}$ were populated by the subsequent $\left({ }^{3} \mathrm{He}, \alpha\right)$ and $\left({ }^{3} \mathrm{He},{ }^{3} \mathrm{He}\right)$ reactions. The intensity of the most important $\gamma$-ray transitions in ${ }^{154-156} \mathrm{Gd}$ as a function of ejectile energy were determined and a sum of non-coincident $\gamma$-rays was formed for the strongest transitions observed from the $\left({ }^{3} \mathrm{He}, \alpha \mathrm{xn}\right)$ and $\left({ }^{3} \mathrm{He},{ }^{3} \mathrm{He}^{\prime} \mathrm{xn}\right)$ channels. From this the probabilities of decay through a given exit channel were derived. When these were compared to decay probabilities from the STAPRE code for the appropriate $(\mathrm{n}, \gamma)$ and $(\mathrm{n}, 2 \mathrm{n})$ decay channel the agreement was found to be remarkable. For a discussion of the "ratio method" and its potential problems see (Escher \& Dietrich 2005).
A more recent experiment (Church et al. 2005) has measured ${ }^{102,104} \mathrm{Ru}\left(\alpha, \alpha^{\prime}\right)$ as surrogate reaction for ${ }^{101,103} \mathrm{Ru}(\mathrm{n}, \gamma)$. An analysis of the experimental data along with a more detailed modeling approach is ongoing (Forssen et al. 2005).

We have noted inconsistencies between our modeled $(\mathrm{n}, 2 \mathrm{n})$ cross sections and measured ones (Frehault 1975) for even-even targets. To check this a modern mesurement of ${ }^{150} \mathrm{Sm}(\mathrm{n}, 2 \mathrm{n}){ }^{149} \mathrm{Sm}$ was conducted (Cooper et al. 2004). It also used measurement of partial gamma-rays as did the Gd experiments of (Bernstein et al. 2005), but with a different comparison to our statistical model results. The experiment was in good agreement with our calculated cross section, which provided confidence in our choice of theoretical models and the development of local systematics for the input to our statistical model codes.

Finally, we note a wide discrepancy in the values of the neutron capture cross section of our principle loaded detector isotope ${ }^{151} \mathrm{Eu}$ (Figure 9, Table 5). This has been measured many times (and often quoted with very small errors). A new experiment has been carried out at LANLCE (LBNL) by the ENP in March of 2004 and 2005 (Agvaanluvsan et al. 2005). Initial yield data has been analysed and we anticipate cross section results in FY06.

This work was performed under the auspices of the U.S. Department of Energy by the University of California Lawrence Livermore National Laboratory under contract W-7405-ENG-48.

\section{REFERENCES}

Agvaanluvsan, U., \& 26 others, "Neutron Capture Cross Section in ${ }^{151}$ Eu", UCRL-ABS-212344, LLNL, 2005

Avrigeanu, M. \& Avrigeanu, V. IPNE-Bucharest Report No. NP-86-1995 (September 1995) http://www.nea.fr/abs/html/iaea0971.html

Avrigeanu, V., Hodgson, P.E., \& Avrigeanu, M. 1994, Phys. Rev. C49, 2136

Bao, Z. Y., Beer, H., Kappeler, F.,, Voss, F., \& Wisshak, K. 2000, Atomic Data \& Nuclear Data Tables, 76, 70

Bauer, R. 2001, private communication.

Beer, H., Voss, F., \& Winters, R. R. 1992, ApJS, 80, 403

Bernstein \& 25 others, "Surrogate Nuclear Reactions using STARS", Intl. Conf. on Nucl. Data 
for Science and Technology, Santa Fe, AIP 769, pg. 890,2005

Bohr, A., \& Mottelson, B. Nuclear Structure Vol. 1, Single-Particle Motion, World Scientific, 1998

Experimental Nuclear Reaction Data File, Brookhaven National Laboratory, US Dept. of Energy, http://www.nndc.bnl.gov/nndc/exfor/

Chadwick, M. 1998, the GNASH statistical model code, http://www.nea.fr/abs/html/psr0125.html

Church, J. et. al. "Determining Neutron Capture Cross Sections with the Surrogate Reaction Technique: Measuring decay probabilities with STARS," 2005, Nucl. Phys. A758 126c$129 \mathrm{c}$.

Cline, C. K. 1972, Nucl. Phys. A195, 353

Cooper, J. R., Becker, J. A., Dashdorj, D., Dietrich, F. S., Garrett, P. E., Hoffman, R., Younes, W., "Measurement of ${ }^{150} \mathrm{Sm}(\mathrm{n}, 2 \mathrm{n} \gamma) 149 \mathrm{Sm}$ Cross Section between Threshold and $20 \mathrm{MeV}$ ", UCRL-TR-205760, LLNL, 2005

A $=150$ Level Scheme, Compilers analysis, ed. N.B. Gove, April 1964, Data Sheets, 5, 1

Dietrich, F.S., 2001, private communication.

ENSDF: Evaluated Nuclear Structure Data File, Brookhaven National Laboratory, US Dept. of Energy, http://www.nndc.bnl.gov/nndc/ensdf/

Escher, J., Ahle, L., Bernstein, L., Church, J.A., Dietrich, F., Forssen, C., and Hoffman, R., "Surrogate nuclear reactions and the origin of the heavy elements", UCRL-PROC-205320, LLNL, 2004

Escher, J., \& Dietrich, F., "Examination of the Validity of the Surrogate Ratio Method for Determining $(\mathrm{n}, \mathrm{f})$ and $(\mathrm{n}, \gamma)$ Cross Sections of Actinides," UCRL-TR-212509-Draft, LLNL, 2005.

Forssen, C. et al. , "Theoretical Challenges of Determining Low-energy Neutron Capture Cross Sections via the Surrogate Technique," 2005, Nucl. Phys. A758 130c-133c.

Frehaut, J., Bertin, A., Bois, R., \& Jary, J. STATUS OF $(\mathrm{N}, 2 \mathrm{~N})$ CROSS SECTION MEASUREMENTS AT BRUYERES-LE-CHATEL (private communication) 1975.
Gardner, D. G. MODEL CALCULATIONS AS ONE MEANS OF SATISFYING THE NEUTRON CROSS SECTION REQUIREMENTS OF THE CTR PROGRAM, NBS Special Publication 425, Washington DC, March 3-7, 1975

Gilbert, A., \& Cameron, A.G.W. 1965, Can. J. Phys., 43, 1446

Demetriou, P., \& Goriely, S. 2001, Nucl. Phys. A695, 95

Hilaire, S., Lagrange, Ch., \& Koning, A. J. 2003, Ann. of Phys. 306, 209

Hofmann, H.M., Richert, J, Tepel, J. W. \& Weidenmuller, H.A. 1975, Ann. of Phys. 90, 403

Hoffman, R. D., Rauscher, T., Woosley, S. E. \& Thielemann, F.-K. 1999, ApJ, 521, 735

Hoffman, R.D., Dietrich, F.S., Bauer, R., Kelley, K., and Mustafa, M., "Neutron and ChargedParticle Induced Cross Sections for Radiochemistry in the Region of Bromine and Krypton", UCRL-TR-205563, LLNL, 2004a

Hoffman, R.D., Dietrich, F.S., Bauer, R., Kelley, K., and Mustafa, M., "Neutron and ChargedParticle Induced Cross Sections for Radiochemistry in the Region of Iodine and Xenon", UCRL-TR-206721, LLNL, 2004b

Iljinov, A.S., Mebel, M.V., Bianchi, N., De Sanctis, E., Guaraldo, C., Lucherini, V., Muccifora, V., Polli, E., Reolon, A.R., \& Rossi, P. 1992, Nucl. Phys. A543, 517

Keisch, B. 1963, Phys. Rev. 129, 769

Koning, A.J., \& Delaroche, J.P. 2003, Nucl. Phys. A713, 231

Kopecky, J., Uhl, M., \& Chrien, R.E. 1993, Phys. Rev. C47, 312

Lohr, J.M. \& Haeberli, W. 1974, Nuc. Phys. A232, 381

McFadden, L., and Satchler, G.R., 1966, Nuc. Phys. 84, 177

Mclane, V., Dunford, C., \& Rose, P. Neutron Cross Sections, Vol. 2, Neutron Cross Section Curves, Academic Press, 1988

Milazzo-Colli, L., and Braga-Marcazzan, G.M., 1973, Nuc. Phys. A210, 297

Moldauer, P. A. 1976 Phys. Rev. C14 764 
Möller, P., Nix, J.R., Myers, W.D., \& Swiatecki, W.J. 1995, Atomic Data \& Nuclear Data Tables, 59, 185

Mughabghab, S. F. and Garber, D. I., Neutron Cross Sections, Brookhaven National Laboratory, 1973, BNL-325/ED3

Mughabghab, S. F., Divadeenam, M., and Holden, N.E., Neutron Cross Sections, 1982, Academic Press, New York

Mustafa, M., Nuclear Data for Science and Technology Proc. 59, p. 274, 1997. Trieste

Nethaway, D.R., Memo, "The Cross-Section Sets Used With the Watusi Program." LLNL ADivision memo, 5 Nov., 1998

Nethaway, D.R., and Mustafa, M. G., "Measured Data Used in the Watusi Cross-Section Sets", UCRL-ID-133269, LLNL, 1999

Nuclear Data Sheets for $\mathrm{A}=150$, ed. C.M. Baglin, 1976, Nuclear Data Sheets, 18, 223

Nuclear Data Sheets for $\mathrm{A}=150$, ed. E. der Mateosian, 1986, Nuclear Data Sheets, 48, 345

Nuclear Data Sheets for $\mathrm{A}=150$, ed. E. der Mateosian \& J.K. Tuli, 1995, Nuclear Data Sheets, 75, 827

Perey, C.M. and Perey, F.G., 1963, Phys. Rev. 132,755

Rauscher, T., Thielemann, F.-K., \& Kratz, K.-L. 1997, Phys. Rev. C., 56, 1613

Rauscher, T., Thielemann, F.-K. 2000, Atomic Data \& Nuclear Data Tables, 79, 47

Rauscher, T., Heger, A. Hoffman, R. D. \& Woosley, S. E. 2002, ApJ, 576, 323

Reffo, G. 1978, ICTP Lecture Series, 17 Jan. - 10 Mar., Trieste

Raynal, J. "ECIS96", Proceedings of the Specialists' Meeting on the Nucleon $\mathrm{Nu}-$ cleus Optical Model up to $200 \mathrm{MeV}, 13-15$ November 1996, Bruyeres-le-Chatel, Franc (http://www.nea.fr/html/science/om200/raynal.pdf)

Handbook for calculations of nuclear reaction data, Reference input parameter library. 1998, IAEATECDOC-1034

Soromel-Stanco et al. IKP Annual Report (unpublished) Juel-Spex-202, p. 54, 1982
Timmes, F. X., Woosley, S. E., \& Weaver, T. A. 1995, ApJS, 98,617

Woosley, S. E., Fowler, W. A., Holmes, J. A. \& Zimmerman, B. A. 1978, Atomic Data \& Nuclear Data Tables, 22, 371

Woosley, S. E., \& Weaver, T. A. 1995, ApJS, 101, 181

Uhl, M., \& Strohmaier, B. IRK-Vienna Report IRK-76/01 1976 (Upd. 1978)

Vonach, H. 1982, UCID-19549, LLNL

Udagawa, T., and Tamura, T. Phys. Rev. C 24, 1348 (1981); 33, 494(1986)

Walter, G. et. al. 1986, Nucl Sci. Eng., 93, 357

West, H., Lanier, R., Mustafa, M., Nuckolls, R., Nagle, R., O'Brien, H., Frehaut, J., Adam, A., and Philis, C., "Some Light-Ion Excitation Fucntion Measurements on Titanium, Yttrium, and Europium, and Associated Results", UCRL-ID-115738, LLNL, 1993

Williams, F. C. Jr. 1970, Phys. Lett. 31B, 184

This 2-column preprint was prepared with the AAS $\mathrm{LAT}_{\mathrm{E}} \mathrm{X}$ macros v5.0. 
A. Basic Nuclear Structure Data

A.1. New Samarium-Europium-Gadolinium Detector Set

Table 9:: Reactions calculated for new cross section sets

\begin{tabular}{|c|c|c|c|c|c|c|c|c|c|c|c|c|c|}
\hline$\overline{{ }^{A} Z}$ & lifetime & $(\mathrm{n}, 2 \mathrm{n})$ & $(\mathrm{n}, 3 \mathrm{n})$ & $\left(\mathrm{n}, \mathrm{n}^{\prime}\right)$ & $(\mathrm{n}, \gamma)$ & $(\mathrm{n}, \mathrm{p})$ & $(\mathrm{n}, \mathrm{np})$ & $(\mathrm{n}, \alpha)$ & $(\mathrm{n}, \mathrm{d})$ & $(\mathrm{p}, \mathrm{n})$ & $(\mathrm{p}, 2 \mathrm{n})$ & $(\mathrm{d}, \mathrm{n})$ & $(\mathrm{d}, 2 \mathrm{n})$ \\
\hline${ }^{144} \mathrm{Sm}$ & $3.07 \%$ & $\bullet$ & $\bullet$ & & $\bullet$ & $\bullet$ & $\bullet$ & $\bullet$ & $\bullet$ & $\bullet$ & $\bullet$ & $\bullet$ & $\bullet$ \\
\hline${ }^{145} \mathrm{Sm}$ & $340 \mathrm{~d}$ & - & - & & - & - & - & - & - & - & - & - & - \\
\hline${ }^{146} \mathrm{Sm}$ & $10.3 \times 10^{7} \mathrm{y}$ & $\bullet$ & $\bullet$ & & $\bullet$ & $\bullet$ & • & • & • & $\bullet$ & $\bullet$ & • & $\bullet$ \\
\hline${ }^{147} \mathrm{Sm}$ & $14.99 \%$ & $\bullet$ & $\bullet$ & & $\bullet$ & $\bullet$ & • & • & $\bullet$ & $\bullet$ & $\bullet$ & $\bullet$ & $\bullet$ \\
\hline${ }^{148} \mathrm{Sm}$ & $11.24 \%$ & $\bullet$ & $\bullet$ & & $\bullet$ & $\bullet$ & • & • & $\bullet$ & $\bullet$ & $\bullet$ & $\bullet$ & $\bullet$ \\
\hline${ }^{149} \mathrm{Sm}$ & $13.82 \%$ & $\bullet$ & $\bullet$ & & • & $\bullet$ & • & • & $\bullet$ & $\bullet$ & - & $\bullet$ & $\bullet$ \\
\hline${ }^{150} \mathrm{Sm}$ & $7.38 \%$ & $\bullet$ & $\bullet$ & & $\bullet$ & $\bullet$ & $\bullet$ & $\bullet$ & $\bullet$ & $\bullet$ & $\bullet$ & $\bullet$ & $\bullet$ \\
\hline${ }^{151} \mathrm{Sm}$ & $90 \mathrm{y}$ & • & • & $\bullet$ & • & $\bullet$ & • & • & $\bullet$ & $\bullet$ & $\bullet$ & $\bullet$ & $\bullet$ \\
\hline${ }^{151} \mathrm{Sm}_{m}$ & $1.4 \mu \mathrm{s}$ & $\bullet$ & $\bullet$ & $\bullet$ & $\bullet$ & $\bullet$ & $\bullet$ & $\bullet$ & $\bullet$ & $\bullet$ & $\bullet$ & $\bullet$ & $\bullet$ \\
\hline${ }^{152} \mathrm{Sm}$ & $26.75 \%$ & • & • & & • & $\bullet$ & $\bullet$ & $\bullet$ & $\bullet$ & $\bullet$ & $\bullet$ & $\bullet$ & $\bullet$ \\
\hline${ }^{153} \mathrm{Sm}$ & $46.284 \mathrm{~h}$ & $\bullet$ & $\bullet$ & $\bullet$ & $\bullet$ & $\bullet$ & $\bullet$ & $\bullet$ & $\bullet$ & $\bullet$ & $\bullet$ & $\bullet$ & $\bullet$ \\
\hline${ }^{153} \mathrm{Sm}_{m}$ & $10.6 \mathrm{~ms}$ & $\bullet$ & $\bullet$ & $\bullet$ & $\bullet$ & $\bullet$ & $\bullet$ & $\bullet$ & $\bullet$ & $\bullet$ & $\bullet$ & $\bullet$ & $\bullet$ \\
\hline${ }^{154} \mathrm{Sm}$ & $22.75 \%$ & • & • & & • & • & $\bullet$ & $\bullet$ & $\bullet$ & $\bullet$ & $\bullet$ & $\bullet$ & $\bullet$ \\
\hline${ }^{155} \mathrm{Sm}$ & $22.3 \mathrm{~m}$ & - & - & & - & - & - & - & - & - & - & - & $\bullet$ \\
\hline${ }^{145} \mathrm{Eu}$ & $5.93 \mathrm{~d}$ & - & $\bullet$ & & $\bullet$ & $\bullet$ & - & $\bullet$ & - & $\bullet$ & - & $\bullet$ & $\bullet$ \\
\hline${ }^{146} \mathrm{Eu}$ & $4.61 \mathrm{~d}$ & $\bullet$ & $\bullet$ & & • & $\bullet$ & $\bullet$ & $\bullet$ & $\bullet$ & $\bullet$ & $\bullet$ & $\bullet$ & $\bullet$ \\
\hline${ }^{147} \mathrm{Eu}$ & $24.1 \mathrm{~d}$ & $\bullet$ & $\bullet$ & & $\bullet$ & $\bullet$ & $\bullet$ & $\bullet$ & $\bullet$ & $\bullet$ & $\bullet$ & $\bullet$ & $\bullet$ \\
\hline${ }^{148} \mathrm{Eu}$ & $54.5 \mathrm{~d}$ & $\bullet$ & $\bullet$ & & • & $\bullet$ & $\bullet$ & $\bullet$ & $\bullet$ & $\bullet$ & $\bullet$ & $\bullet$ & $\bullet$ \\
\hline${ }^{149} \mathrm{Eu}$ & $93.1 \mathrm{~d}$ & $\bullet$ & $\bullet$ & $\bullet$ & • & $\bullet$ & $\bullet$ & $\bullet$ & $\bullet$ & $\bullet$ & $\bullet$ & $\bullet$ & $\bullet$ \\
\hline${ }^{149} \mathrm{Eu}_{m}$ & $2.45 \mu \mathrm{s}$ & $\bullet$ & $\bullet$ & $\bullet$ & $\bullet$ & $\bullet$ & $\bullet$ & $\bullet$ & $\bullet$ & $\bullet$ & $\bullet$ & $\bullet$ & $\bullet$ \\
\hline${ }^{150} \mathrm{Eu}$ & $36.9 \mathrm{y}$ & • & • & $\bullet$ & • & $\bullet$ & $\bullet$ & $\bullet$ & $\bullet$ & $\bullet$ & $\bullet$ & $\bullet$ & $\bullet$ \\
\hline${ }^{150} \mathrm{Eu}_{m}$ & $12.8 \mathrm{~h}$ & $\bullet$ & $\bullet$ & $\bullet$ & $\bullet$ & $\bullet$ & $\bullet$ & $\bullet$ & $\bullet$ & $\bullet$ & $\bullet$ & $\bullet$ & $\bullet$ \\
\hline${ }^{151} \mathrm{Eu}$ & $47.81 \%$ & $\bullet$ & $\bullet$ & $\bullet$ & $\bullet$ & $\bullet$ & $\bullet$ & $\bullet$ & $\bullet$ & $\bullet$ & $\bullet$ & $\bullet$ & $\bullet$ \\
\hline${ }^{151} \mathrm{Eu}_{m}$ & $58.9 \mu \mathrm{s}$ & $\bullet$ & $\bullet$ & $\bullet$ & $\bullet$ & $\bullet$ & $\bullet$ & $\bullet$ & $\bullet$ & $\bullet$ & $\bullet$ & $\bullet$ & $\bullet$ \\
\hline${ }^{152} \mathrm{Eu}$ & $13.52 \mathrm{y}$ & $\bullet$ & $\bullet$ & $\bullet$ & $\bullet$ & $\bullet$ & $\bullet$ & $\bullet$ & $\bullet$ & $\bullet$ & $\bullet$ & $\bullet$ & $\bullet$ \\
\hline${ }^{152} \mathrm{Eu}_{m 1}$ & $9.31 \mathrm{~h}$ & • & $\bullet$ & $\bullet$ & $\bullet$ & $\bullet$ & $\bullet$ & $\bullet$ & $\bullet$ & $\bullet$ & $\bullet$ & $\bullet$ & $\bullet$ \\
\hline${ }^{152} \mathrm{Eu}_{m 2}$ & $96 \mathrm{~m}$ & $\bullet$ & $\bullet$ & $\bullet$ & $\bullet$ & $\bullet$ & $\bullet$ & $\bullet$ & $\bullet$ & $\bullet$ & $\bullet$ & $\bullet$ & $\bullet$ \\
\hline${ }^{153} \mathrm{Eu}$ & $52.19 \%$ & $\bullet$ & $\bullet$ & & $\bullet$ & $\bullet$ & $\bullet$ & $\bullet$ & - & $\bullet$ & - & $\bullet$ & - \\
\hline${ }^{154} \mathrm{Eu}$ & $8.592 \mathrm{y}$ & $\bullet$ & $\bullet$ & $\bullet$ & - & - & - & $\bullet$ & - & $\bullet$ & - & - & - \\
\hline${ }^{154} \mathrm{Eu}_{m 1}$ & $2.2 \mu \mathrm{s}$ & $\bullet$ & $\bullet$ & $\bullet$ & $\bullet$ & $\bullet$ & $\bullet$ & $\bullet$ & $\bullet$ & $\bullet$ & $\bullet$ & $\bullet$ & $\bullet$ \\
\hline${ }^{154} \mathrm{Eu}_{m 2}$ & $46.3 \mathrm{~m}$ & $\bullet$ & $\bullet$ & $\bullet$ & $\bullet$ & $\bullet$ & $\bullet$ & $\bullet$ & $\bullet$ & $\bullet$ & $\bullet$ & $\bullet$ & $\bullet$ \\
\hline${ }^{155} \mathrm{Eu}$ & $4.761 \mathrm{y}$ & $\bullet$ & $\bullet$ & & $\bullet$ & $\bullet$ & $\bullet$ & $\bullet$ & $\bullet$ & $\bullet$ & - & - & - \\
\hline${ }^{156} \mathrm{Eu}$ & $15.19 \mathrm{~d}$ & $\bullet$ & $\bullet$ & & $\bullet$ & $\bullet$ & $\bullet$ & $\bullet$ & $\bullet$ & $\bullet$ & - & - & - \\
\hline${ }^{146} \mathrm{Gd}$ & $48.27 \mathrm{~d}$ & $\bullet$ & $\bullet$ & & - & - & - & - & - & - & $\bullet$ & $\bullet$ & $\bullet$ \\
\hline${ }^{147} \mathrm{Gd}$ & $38.06 \mathrm{~h}$ & $\bullet$ & - & & - & - & - & - & - & - & - & - & - \\
\hline${ }^{148} \mathrm{Gd}$ & 74.6 y & $\bullet$ & $\bullet$ & & $\bullet$ & $\bullet$ & $\bullet$ & $\bullet$ & $\bullet$ & $\bullet$ & $\bullet$ & $\bullet$ & $\bullet$ \\
\hline${ }^{149} \mathrm{Gd}$ & $9.28 \mathrm{~d}$ & $\bullet$ & $\bullet$ & & $\bullet$ & $\bullet$ & $\bullet$ & $\bullet$ & $\bullet$ & $\bullet$ & $\bullet$ & $\bullet$ & $\bullet$ \\
\hline${ }^{150} \mathrm{Gd}$ & $1.79 \times 10^{6} \mathrm{y}$ & $\bullet$ & - & & - & - & - & - & - & - & - & - & $\bullet$ \\
\hline${ }^{151} \mathrm{Gd}$ & $124 \mathrm{~d}$ & - & - & & - & - & - & - & - & - & - & - & $\bullet$ \\
\hline${ }^{152} \mathrm{Gd}$ & $0.20 \%$ & $\bullet$ & $\bullet$ & & $\bullet$ & $\bullet$ & $\bullet$ & $\bullet$ & $\bullet$ & $\bullet$ & $\bullet$ & $\bullet$ & $\bullet$ \\
\hline${ }^{153} \mathrm{Gd}$ & $240.4 \mathrm{~d}$ & $\bullet$ & $\bullet$ & $\bullet$ & $\bullet$ & $\bullet$ & $\bullet$ & $\bullet$ & $\bullet$ & $\bullet$ & $\bullet$ & $\bullet$ & $\bullet$ \\
\hline${ }^{153} \mathrm{Gd}_{m 1}$ & $3.5 \mu \mathrm{s}$ & $\bullet$ & $\bullet$ & $\bullet$ & $\bullet$ & $\bullet$ & $\bullet$ & $\bullet$ & $\bullet$ & $\bullet$ & $\bullet$ & $\bullet$ & $\bullet$ \\
\hline${ }^{153} \mathrm{Gd}_{m 2}$ & $76 \mu \mathrm{s}$ & $\bullet$ & $\bullet$ & - & $\bullet$ & - & - & - & - & - & - & - & - \\
\hline${ }^{154} \mathrm{Gd}$ & $2.18 \%$ & $\bullet$ & $\bullet$ & & $\bullet$ & $\bullet$ & $\bullet$ & $\bullet$ & $\bullet$ & $\bullet$ & - & - & - \\
\hline${ }^{155} \mathrm{Gd}$ & $14.80 \%$ & $\bullet$ & $\bullet$ & $\bullet$ & $\bullet$ & $\bullet$ & $\bullet$ & $\bullet$ & $\bullet$ & $\bullet$ & $\bullet$ & $\bullet$ & $\bullet$ \\
\hline${ }^{155} \mathrm{Gd}_{m}$ & $32 \mathrm{~ms}$ & - & - & - & - & $\bullet$ & $\bullet$ & $\bullet$ & $\bullet$ & - & $\bullet$ & $\bullet$ & $\bullet$ \\
\hline${ }^{156} \mathrm{Gd}$ & $20.47 \%$ & - & - & & - & - & - & - & - & - & - & $\bullet$ & $\bullet$ \\
\hline
\end{tabular}


Table 9: (continued)

\begin{tabular}{|c|c|c|c|c|c|c|c|c|c|c|c|c|}
\hline${ }^{A} Z$ & lifetime & $(\mathrm{n}, 2 \mathrm{n})$ & $\overline{(\mathrm{n}, 3 \mathrm{n})\left(\mathrm{n}, \mathrm{n}^{\prime}\right)}$ & $(\mathrm{n}, \gamma)$ & $(\mathrm{n}, \mathrm{p})$ & $(\mathrm{n}, \mathrm{np})$ & $(\mathrm{n}, \alpha)$ & $(\mathrm{n}, \mathrm{d})$ & $(\mathrm{p}, \mathrm{n})$ & $(p, 2 n)$ & $(\mathrm{d}, \mathrm{n})$ & $(\mathrm{d}, 2 \mathrm{n})$ \\
\hline${ }^{157} \mathrm{Gd}$ & $15.65 \%$ & $\bullet$ & $\bullet$ & $\bullet$ & $\bullet$ & $\bullet$ & $\bullet$ & $\bullet$ & $\bullet$ & $\bullet$ & $\bullet$ & - \\
\hline${ }^{158} \mathrm{Gd}$ & $24.84 \%$ & $\bullet$ & • & • & $\bullet$ & • & $\bullet$ & $\bullet$ & - & - & • & - \\
\hline${ }^{160} \mathrm{Gd}$ & $21.86 \%$ & • & $\bullet$ & $\bullet$ & $\bullet$ & $\bullet$ & $\bullet$ & $\bullet$ & $\bullet$ & - & & \\
\hline
\end{tabular}




\section{A.2. Binding and Separation Energies}

Table 10:: Spins, parities, binding energies, and separation energies for new samarium and europium sets

\begin{tabular}{|c|c|c|c|c|c|c|}
\hline${ }^{A} Z$ & $J^{\pi}$ & $\mathrm{BE}(\mathrm{MeV})$ & $S_{n}(\mathrm{MeV})$ & $S_{p}(\mathrm{MeV})$ & $S_{\alpha}(\mathrm{MeV})$ & $\overline{S_{d}(\mathrm{MeV})}$ \\
\hline${ }^{144} \mathrm{Sm}$ & $0+$ & 1195.74 & 10.52 & 6.29 & -0.08 & 14.03 \\
\hline${ }^{145} \mathrm{Sm}$ & $7 / 2-$ & 1202.50 & 6.76 & 6.53 & -1.12 & 10.83 \\
\hline${ }^{146} \mathrm{Sm}$ & $0+$ & 1210.91 & 8.41 & 7.02 & -2.53 & 12.72 \\
\hline${ }^{147} \mathrm{Sm}$ & $7 / 2-$ & 1217.26 & 6.34 & 7.10 & -2.31 & 11.13 \\
\hline${ }^{148} \mathrm{Sm}$ & $0+$ & 1225.40 & 8.14 & 7.58 & -1.99 & 13.02 \\
\hline${ }^{149} \mathrm{Sm}$ & $7 / 2-$ & 1231.27 & 5.87 & 7.56 & -1.87 & 11.23 \\
\hline${ }^{150} \mathrm{Sm}$ & $0+$ & 1239.25 & 7.99 & 8.28 & -1.45 & 13.32 \\
\hline${ }^{151} \mathrm{Sm}$ & $5 / 2-$ & 1244.85 & 5.60 & 8.27 & -1.15 & 11.65 \\
\hline${ }^{151} \mathrm{Sm}_{m}$ & $11 / 2$ & $(-261 \mathrm{keV})$ & & & & \\
\hline${ }^{152} \mathrm{Sm}$ & $0+$ & 1253.11 & 8.26 & 8.66 & -0.22 & 14.30 \\
\hline${ }^{153} \mathrm{Sm}$ & $3 / 2+$ & 1258.98 & 5.87 & 8.59 & 0.61 & 12.31 \\
\hline${ }^{153} \mathrm{Sm}_{m}$ & $11 / 2$ & $(-98 \mathrm{keV})$ & & & & \\
\hline${ }^{154} \mathrm{Sm}$ & $0+$ & 1266.94 & 7.97 & 9.07 & 1.19 & 14.33 \\
\hline${ }^{155} \mathrm{Sm}$ & $3 / 2-$ & 1272.75 & 5.81 & 9.07 & 1.67 & 12.65 \\
\hline${ }^{156} \mathrm{Sm}$ & $0+$ & 1279.99 & 7.24 & 9.68 & 1.63 & 14.09 \\
\hline${ }^{145} \mathrm{Eu}$ & $5 / 2+$ & 1199.06 & 10.41 & 3.32 & -0.05 & 11.61 \\
\hline${ }^{146} \mathrm{Eu}$ & 4- & 1206.25 & 7.20 & 3.76 & -1.53 & 8.29 \\
\hline${ }^{147} \mathrm{Eu}$ & $5 / 2+$ & 1214.75 & 8.50 & 3.84 & -2.99 & 10.03 \\
\hline${ }^{148} \mathrm{Eu}$ & $5-$ & 1221.51 & 6.76 & 4.25 & -2.76 & 8.37 \\
\hline${ }^{149} \mathrm{Eu}$ & $5 / 2+$ & 1229.79 & 8.28 & 4.39 & -2.40 & 10.31 \\
\hline${ }^{149} \mathrm{Eu}_{m}$ & $11 / 2$ & $(-496 \mathrm{keV})$ & & & & \\
\hline${ }^{150} \mathrm{Eu}$ & $5-$ & 1236.21 & 6.42 & 4.94 & -2.24 & 8.59 \\
\hline${ }^{150} \mathrm{Eu}_{m}$ & $0-$ & $(-42 \mathrm{keV})$ & & & & \\
\hline${ }^{151} \mathrm{Eu}$ & $5 / 2+$ & 1244.14 & 7.93 & 4.89 & -1.97 & 10.65 \\
\hline${ }^{151} \mathrm{Eu}_{m}$ & $11 / 2$ & $(-196 \mathrm{keV})$ & & & & \\
\hline${ }^{152} \mathrm{Eu}$ & $3-$ & 1250.45 & 6.31 & 5.60 & -1.56 & 8.97 \\
\hline${ }^{152} \mathrm{Eu}_{m 1}$ & $0-$ & $(-46 \mathrm{keV})$ & & & & \\
\hline${ }^{152} \mathrm{Eu}_{m 2}$ & 8- & $(-148 \mathrm{keV})$ & & & & \\
\hline${ }^{153} \mathrm{Eu}$ & $5 / 2+$ & 1259.00 & 8.55 & 5.89 & -0.28 & 11.93 \\
\hline${ }^{154} \mathrm{Eu}$ & $3-$ & 1265.44 & 6.44 & 6.47 & 0.56 & 10.11 \\
\hline${ }^{154} \mathrm{Eu}_{m 1}$ & $2+$ & $(-68 \mathrm{keV})$ & & & & \\
\hline${ }^{154} \mathrm{Eu}_{m 2}$ & 8- & $(-145 \mathrm{keV})$ & & & & \\
\hline${ }^{155} \mathrm{Eu}$ & $5 / 2+$ & 1273.59 & 8.15 & 6.65 & 0.85 & 12.39 \\
\hline${ }^{156} \mathrm{Eu}$ & $0+$ & 1279.93 & 6.34 & 7.18 & 1.24 & 10.76 \\
\hline${ }^{157} \mathrm{Eu}$ & $5 / 2+$ & 1287.38 & 7.45 & 7.39 & 1.21 & 12.41 \\
\hline${ }^{146} \mathrm{Gd}$ & $0+$ & 1204.44 & 11.22 & 5.39 & -0.47 & 13.57 \\
\hline${ }^{147} \mathrm{Gd}$ & $7 / 2-$ & 1211.78 & 7.34 & 5.53 & -1.74 & 10.50 \\
\hline${ }^{148} \mathrm{Gd}$ & $0+$ & 1220.77 & 8.98 & 6.01 & -3.27 & 12.29 \\
\hline${ }^{149} \mathrm{Gd}$ & $7 / 2-$ & 1227.69 & 6.93 & 6.19 & -3.10 & 10.72 \\
\hline${ }^{150} \mathrm{Gd}$ & $0+$ & 1236.40 & 8.71 & 6.61 & -2.81 & 12.67 \\
\hline${ }^{151} \mathrm{Gd}$ & $7 / 2-$ & 1242.90 & 6.50 & 6.69 & -2.65 & 10.88 \\
\hline${ }^{152} \mathrm{Gd}$ & $0+$ & 1251.49 & 8.59 & 7.34 & -2.21 & 13.05 \\
\hline${ }^{153} \mathrm{Gd}$ & $3 / 2-$ & 1257.73 & 6.25 & 7.28 & -1.83 & 11.37 \\
\hline${ }^{153} \mathrm{Gd}_{m 1}$ & $9 / 2+$ & $(-95 \mathrm{keV})$ & & & & \\
\hline${ }^{153} \mathrm{Gd}_{m 2}$ & $11 / 2$ & $(-171 \mathrm{keV})$ & & & & \\
\hline${ }^{154} \mathrm{Gd}$ & $0+$ & 1266.63 & 8.89 & 7.63 & -0.92 & 13.95 \\
\hline${ }^{155} \mathrm{Gd}$ & $3 / 2-$ & 1273.06 & 6.44 & 7.62 & -0.08 & 11.84 \\
\hline${ }^{155} \mathrm{Gd}_{m}$ & $11 / 2$ & $(-121 \mathrm{keV})$ & & & & \\
\hline
\end{tabular}


Table 10: (continued)

\begin{tabular}{lcccccc}
\hline \hline${ }^{A} Z$ & $J^{\pi}$ & $\mathrm{BE}(\mathrm{MeV})$ & $S_{n}(\mathrm{MeV})$ & $S_{p}(\mathrm{MeV})$ & $S_{\alpha}(\mathrm{MeV})$ & $S_{d}(\mathrm{MeV})$ \\
\hline${ }^{156} \mathrm{Gd}$ & $0+$ & 1281.60 & 8.54 & 8.01 & 0.20 & 13.93 \\
${ }^{157} \mathrm{Gd}$ & $3 / 2-$ & 1287.96 & 6.36 & 8.03 & 0.69 & 12.14 \\
${ }^{158} \mathrm{Gd}$ & $0+$ & 1295.90 & 7.94 & 8.52 & 0.66 & 13.74 \\
${ }^{159} \mathrm{Gd}$ & $3 / 2-$ & 1301.84 & 5.94 & 8.64 & 0.80 & 12.24 \\
${ }^{160} \mathrm{Gd}$ & $0+$ & 1309.29 & 7.45 & 9.18 & 1.00 & 13.87 \\
${ }^{161} \mathrm{Gd}$ & $5 / 2-$ & 1314.93 & 5.64 & 9.43 & 1.20 & 12.59 \\
\hline
\end{tabular}




\section{A.3. Q-values for Select Reactions}

Table 11:: Q-values $(\mathrm{MeV})$ for select reactions in the new samarium and europium sets

\begin{tabular}{|c|c|c|c|c|c|c|c|c|c|c|}
\hline Target & $(\mathrm{n}, 2 \mathrm{n})$ & $(\mathrm{n}, 3 \mathrm{n})$ & $(\mathrm{n}, \mathrm{p})$ & $(\mathrm{n}, \mathrm{np})$ & $(\mathrm{n}, \mathrm{a})$ & $(\mathrm{n}, \mathrm{d})$ & $(\mathrm{p}, \mathrm{n})$ & $(\mathrm{p}, 2 \mathrm{n})$ & $(\mathrm{d}, \mathrm{n})$ & $(\mathrm{d}, 2 \mathrm{n})$ \\
\hline${ }^{144} \mathrm{Sm}$ & -10.52 & -19.12 & 0.23 & -6.29 & 7.87 & -4.07 & -7.10 & -16.58 & 1.09 & -9.32 \\
\hline${ }^{145} \mathrm{Sm}$ & -6.76 & -17.28 & 1.40 & -6.53 & 10.94 & -4.30 & -3.44 & -13.85 & 1.53 & -5.67 \\
\hline${ }^{146} \mathrm{Sm}$ & -8.41 & -15.17 & -0.76 & -7.02 & 8.65 & -4.79 & -4.66 & -11.86 & 1.61 & -6.88 \\
\hline${ }^{147} \mathrm{Sm}$ & -6.34 & -14.76 & 0.56 & -7.10 & 10.13 & -4.88 & -2.50 & -11.00 & 2.03 & -4.73 \\
\hline${ }^{148} \mathrm{Sm}$ & -8.14 & -14.48 & -1.69 & -7.58 & 7.74 & -5.36 & -3.89 & -10.65 & 2.17 & -6.11 \\
\hline${ }^{149} \mathrm{Sm}$ & -5.87 & -14.01 & -0.29 & -7.56 & 9.43 & -5.33 & -1.48 & -9.76 & 2.72 & -3.70 \\
\hline${ }^{150} \mathrm{Sm}$ & -7.99 & -13.86 & -2.67 & -8.28 & 6.74 & -6.05 & -3.04 & -9.46 & 2.67 & -5.27 \\
\hline${ }^{151} \mathrm{Sm}$ & -5.60 & -13.58 & -0.41 & -8.27 & 8.48 & -6.04 & -0.71 & -8.64 & 3.38 & -2.93 \\
\hline${ }^{152} \mathrm{Sm}$ & -8.26 & -13.85 & -2.72 & -8.66 & 5.26 & -6.44 & -2.66 & -8.96 & 3.67 & -4.88 \\
\hline${ }^{153} \mathrm{Sm}$ & -5.87 & -14.13 & -1.10 & -8.59 & 6.77 & -6.36 & 0.03 & -8.52 & 4.24 & -2.20 \\
\hline${ }^{154} \mathrm{Sm}$ & -7.97 & -13.83 & -3.26 & -9.07 & 4.14 & -6.84 & -1.50 & -7.94 & 4.43 & -3.72 \\
\hline${ }^{155} \mathrm{Sm}$ & -5.81 & -13.77 & -2.44 & -9.07 & 5.61 & -6.85 & 0.84 & -7.31 & 4.96 & -1.38 \\
\hline${ }^{145} \mathrm{Eu}$ & -10.41 & $\begin{array}{l}-19.89 \\
\end{array}$ & 3.44 & $\begin{array}{l}-3.32 \\
\end{array}$ & 8.73 & $\begin{array}{c}-1.09 \\
\end{array}$ & -5.84 & -14.94 & 3.16 & -8.06 \\
\hline${ }^{146} \mathrm{Eu}$ & -7.20 & -17.61 & 4.66 & -3.76 & 11.49 & -1.53 & -1.81 & -13.03 & 3.30 & -4.04 \\
\hline${ }^{147} \mathrm{Eu}$ & -8.50 & -15.69 & 2.50 & -3.84 & 9.52 & -1.61 & -2.97 & -10.31 & 3.79 & -5.19 \\
\hline${ }^{148} \mathrm{Eu}$ & -6.76 & -15.25 & 3.89 & -4.25 & 10.69 & -2.03 & -0.74 & -9.72 & 3.96 & -2.97 \\
\hline${ }^{149} \mathrm{Eu}$ & -8.28 & -15.04 & 1.48 & -4.39 & 8.66 & -2.17 & -2.10 & -9.03 & 4.39 & -4.32 \\
\hline${ }^{150} \mathrm{Eu}$ & -6.42 & -14.70 & 3.04 & -4.94 & 9.90 & -2.72 & 0.19 & -8.52 & 4.46 & -2.04 \\
\hline${ }^{151} \mathrm{Eu}$ & -7.93 & -14.35 & 0.71 & -4.89 & 7.86 & -2.67 & -1.25 & -7.74 & 5.12 & -3.47 \\
\hline${ }^{152} \mathrm{Eu}$ & -6.31 & -14.24 & 2.66 & -5.60 & 8.82 & -3.38 & 1.04 & -7.55 & 5.06 & -1.19 \\
\hline${ }^{153} \mathrm{Eu}$ & -8.55 & -14.86 & -0.03 & -5.89 & 5.88 & -3.67 & -1.27 & -7.51 & 5.40 & -3.49 \\
\hline${ }^{154} \mathrm{Eu}$ & -6.44 & -14.99 & 1.50 & -6.47 & 7.30 & -4.24 & 1.19 & -7.71 & 5.40 & -1.04 \\
\hline${ }^{155} \mathrm{Eu}$ & -8.15 & -14.59 & -0.84 & -6.65 & 5.09 & -4.43 & -0.53 & -6.97 & 5.78 & -2.75 \\
\hline${ }^{156} \mathrm{Eu}$ & -6.34 & -14.49 & 0.06 & -7.18 & 6.24 & -4.96 & 1.67 & -6.87 & 5.80 & -0.56 \\
\hline${ }^{146} \mathrm{Gd}$ & -11.22 & -20.32 & 1.81 & -5.39 & 9.08 & -3.16 & -9.05 & -18.70 & -0.28 & -11.28 \\
\hline${ }^{147} \mathrm{Gd}$ & -7.34 & -18.56 & 2.97 & -5.53 & 12.25 & -3.30 & -5.39 & -16.39 & 0.21 & -7.62 \\
\hline${ }^{148} \mathrm{Gd}$ & -8.98 & -16.32 & 0.74 & -6.01 & 10.03 & -3.79 & -6.55 & -14.38 & 0.28 & -8.77 \\
\hline${ }^{149} \mathrm{Gd}$ & -6.93 & -15.91 & 2.10 & -6.19 & 11.51 & -3.96 & -4.42 & -13.47 & 1.04 & -6.64 \\
\hline${ }^{150} \mathrm{Gd}$ & -8.71 & -15.64 & -0.19 & -6.61 & 9.15 & -4.39 & -5.44 & -13.13 & 0.93 & -7.66 \\
\hline${ }^{151} \mathrm{Gd}$ & -6.50 & -15.20 & 1.25 & -6.69 & 10.79 & -4.46 & -3.35 & -11.94 & 1.60 & -5.57 \\
\hline${ }^{152} \mathrm{Gd}$ & -8.59 & -15.09 & -1.04 & -7.34 & 8.08 & -5.12 & -4.77 & -11.94 & 1.67 & -6.99 \\
\hline${ }^{153} \mathrm{Gd}$ & -6.25 & -14.84 & 1.27 & -7.28 & 9.81 & -5.06 & -2.35 & -11.02 & 2.33 & -4.58 \\
\hline${ }^{154} \mathrm{Gd}$ & -8.89 & -15.14 & -1.19 & -7.63 & 6.52 & -5.40 & -4.34 & -11.25 & 2.61 & -6.56 \\
\hline${ }^{155} \mathrm{Gd}$ & -6.44 & -15.33 & 0.53 & -7.62 & 8.34 & -5.40 & -1.60 & -10.77 & 3.08 & -3.83 \\
\hline${ }^{156} \mathrm{Gd}$ & -8.54 & -14.97 & -1.67 & -8.01 & 5.67 & -5.78 & -3.23 & -10.14 & 3.29 & -5.45 \\
\hline${ }^{157} \mathrm{Gd}$ & -6.36 & -14.90 & -0.58 & -8.03 & 7.28 & -5.80 & -0.84 & -9.59 & 3.71 & -3.07 \\
\hline${ }^{158} \mathrm{Gd}$ & -7.94 & -14.30 & -2.70 & -8.52 & 5.15 & -6.29 & -2.00 & -8.78 & 3.91 & -4.23 \\
\hline${ }^{159} \mathrm{Gd}$ & -5.94 & -13.88 & -1.73 & -8.64 & 6.45 & -6.42 & 0.19 & -7.95 & 4.34 & -2.04 \\
\hline${ }^{160} \mathrm{Gd}$ & -7.45 & -13.40 & -3.79 & -9.18 & 4.43 & -6.96 & -0.89 & -7.26 & 4.58 & -3.11 \\
\hline
\end{tabular}




\section{A.4. Adopted Level Schemes}

In Figure 20 we present the nuclear energy level diagrams for the isotopes of samarium, europium, and gadolinium with $81 \leq N \leq 94$. Select level diagrams for the nuclei of $\mathrm{Nd}$, Pm, and $\mathrm{Tb}$ that also were modified can be obtained from the authors on request. All other level schemes were adopted from the RIPL data file BUDAPEST.DAT (RIPL 1998). The level and transition line energies are in keV, the branching ratios from the initial state are given in \%. Only the first twenty levels are shown in the diagrams. For some nuclei more levels than those shown were used, the number $(\mathrm{N})$ is given in the level density parameter tables (12). 


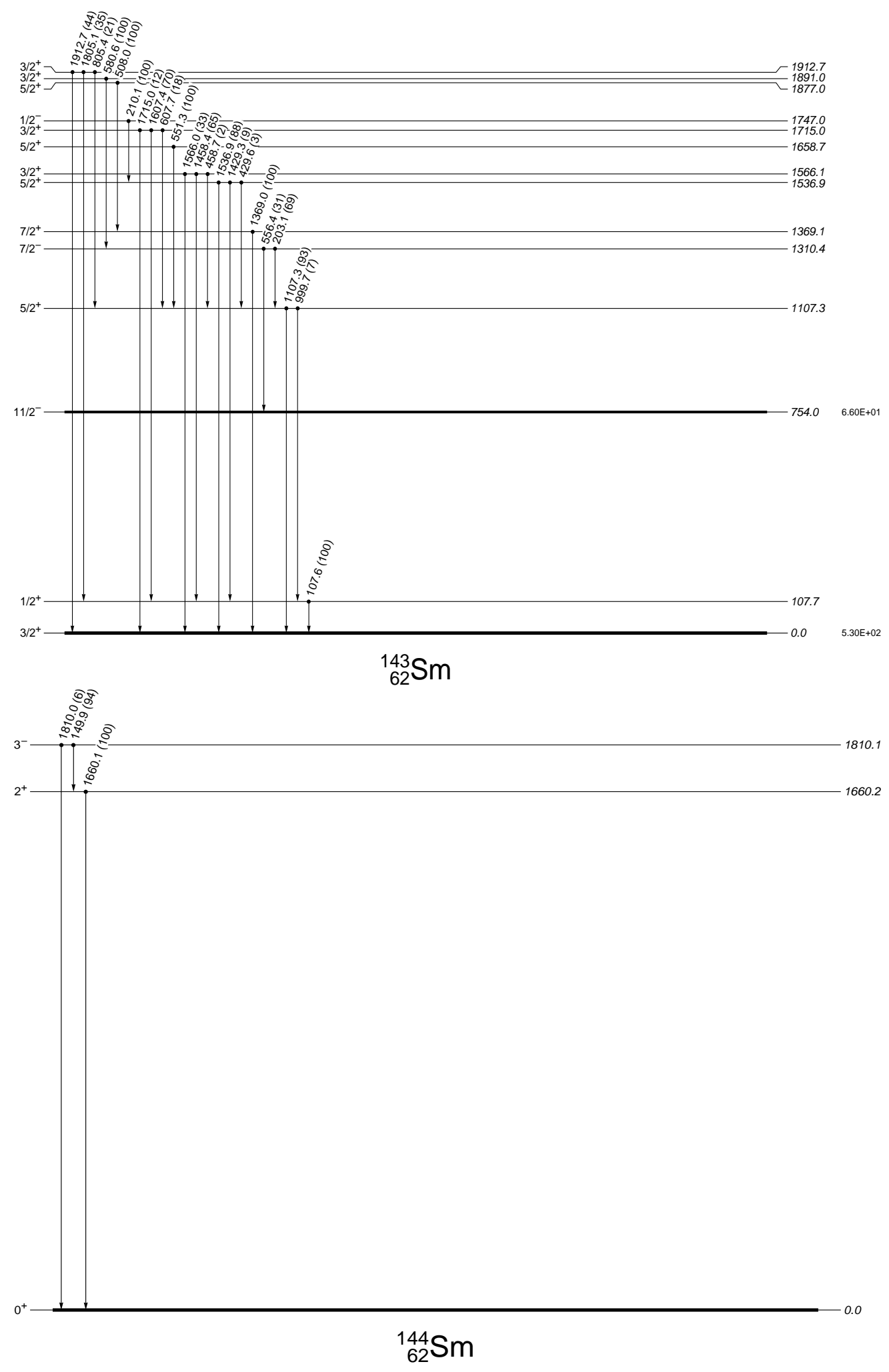

Fig. 20.- Adopted level schemes for Sm, Eu, and Gd nuclei. 

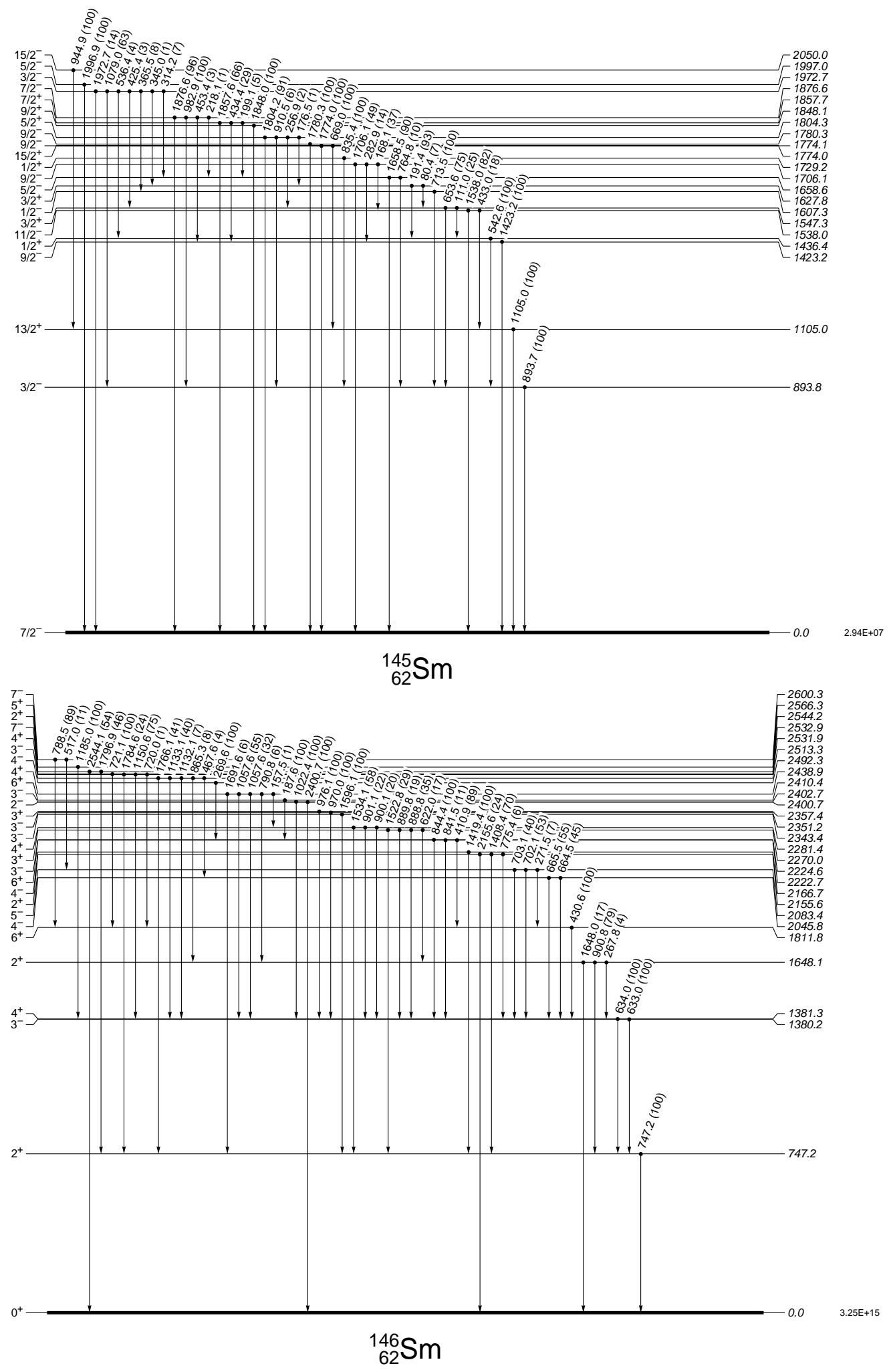

Fig. 20.- (continued) 

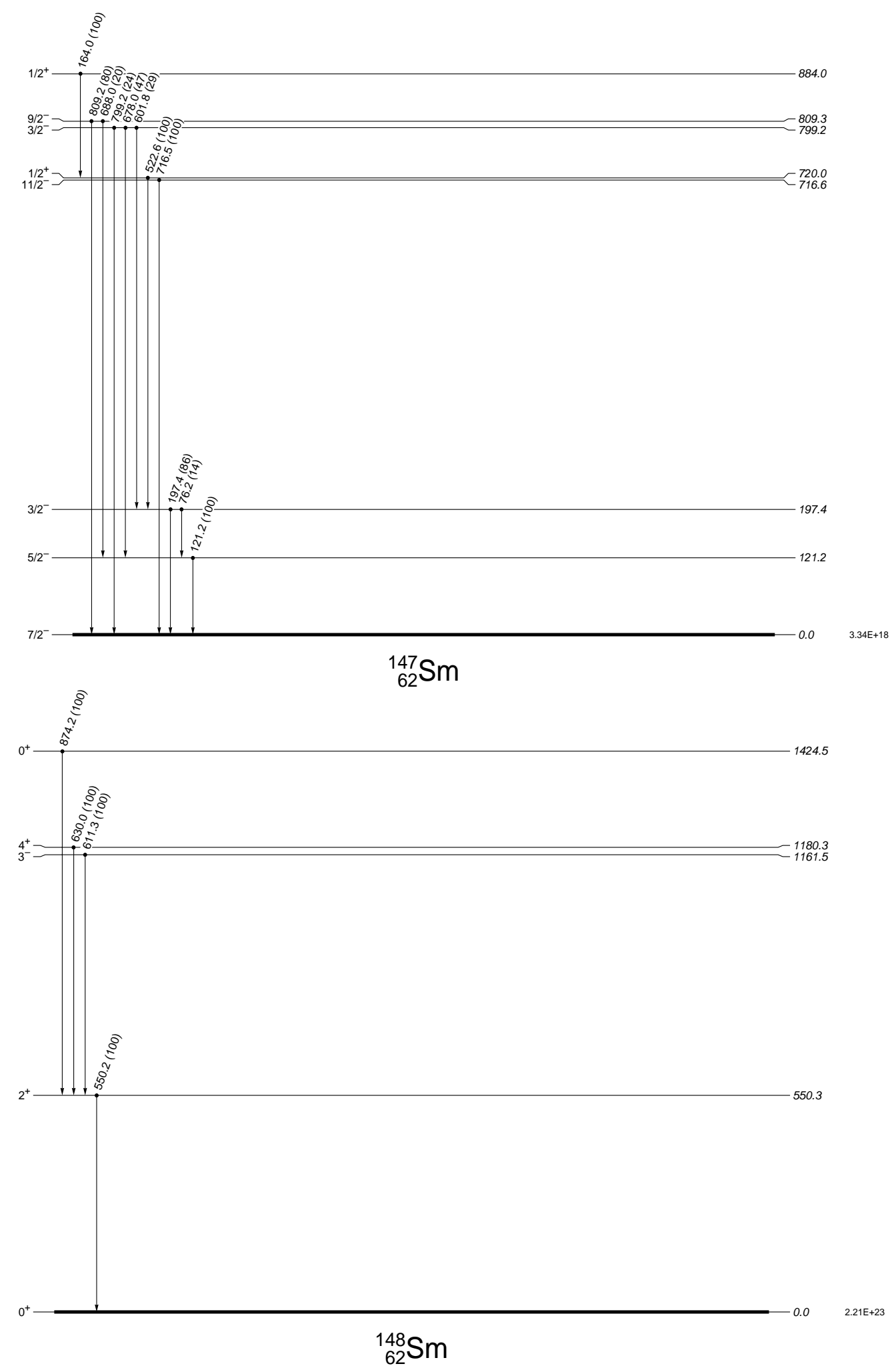

Fig. 20.- (continued) 

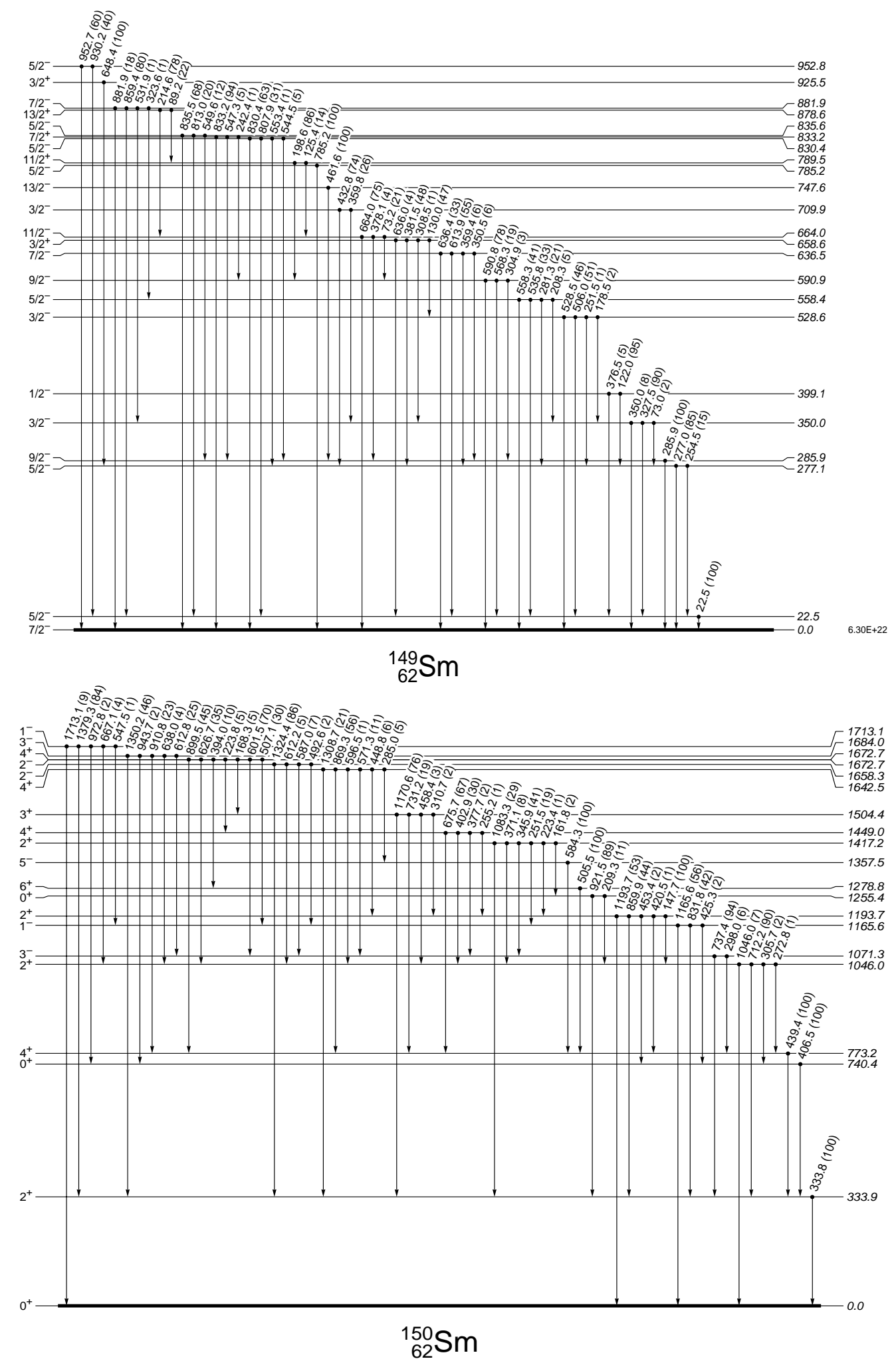

Fig. 20.- (continued) 

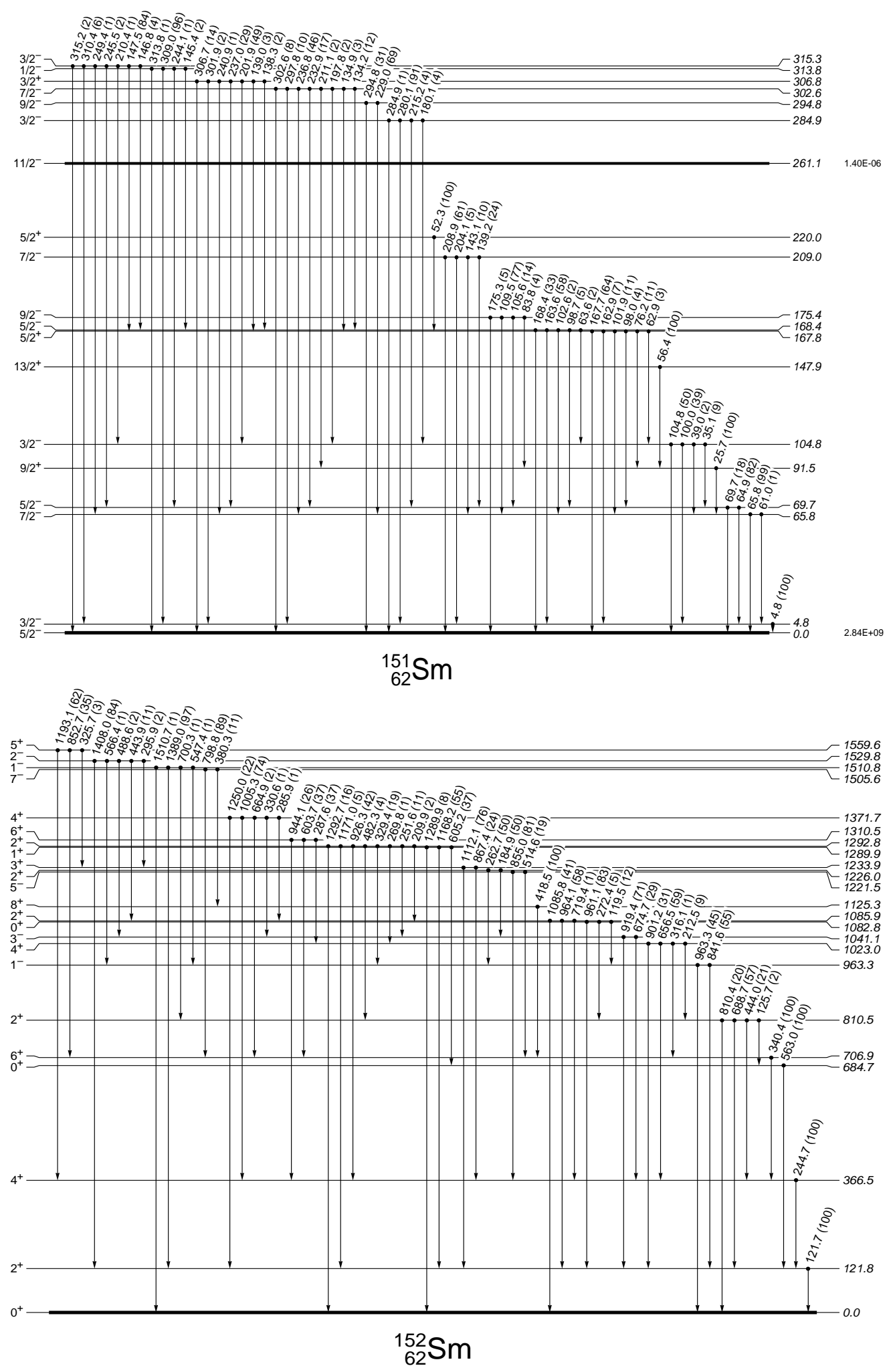

Fig. 20.- (continued) 

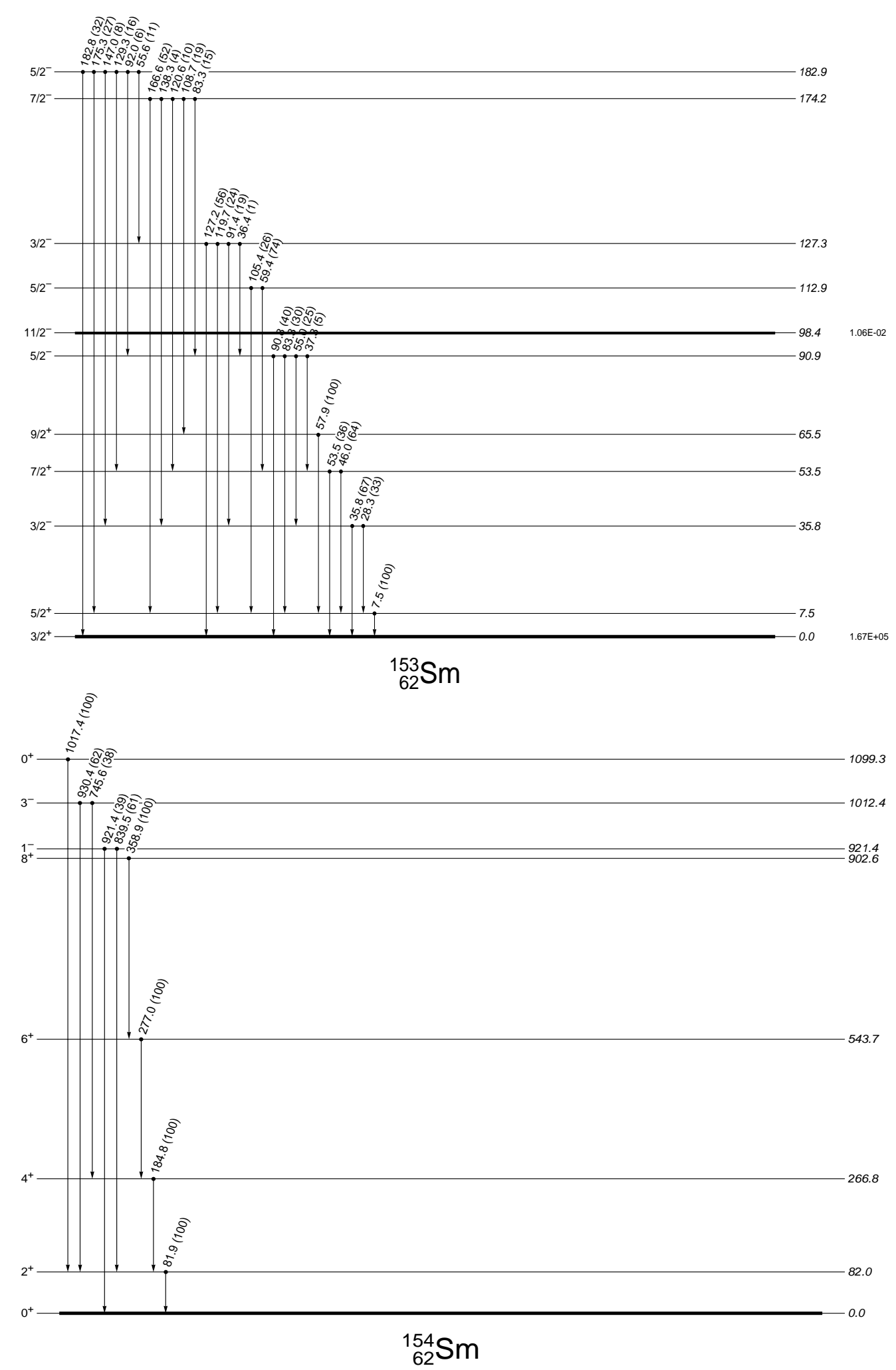

Fig. 20.- (continued) 

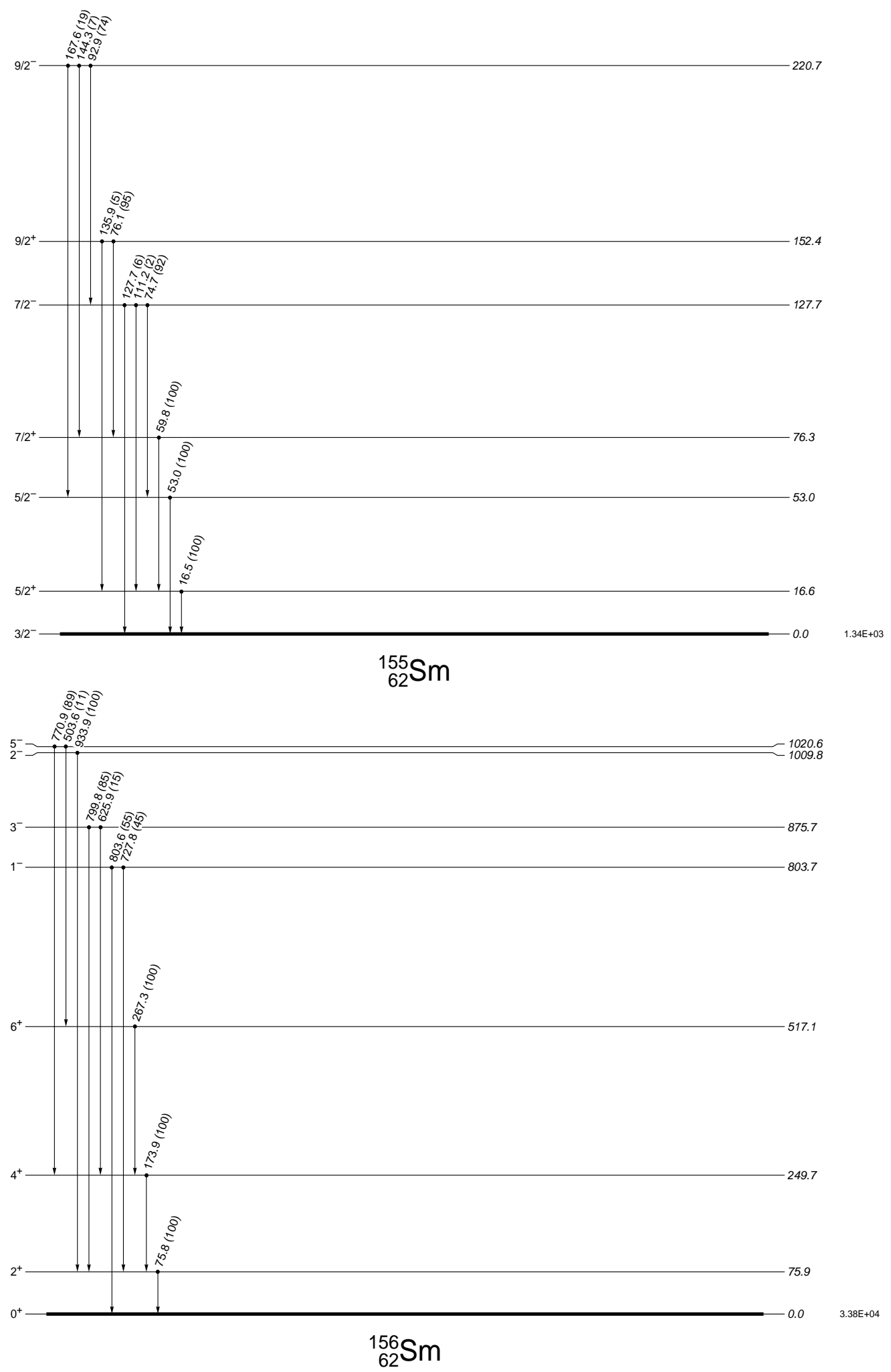

Fig. 20.- (continued) 

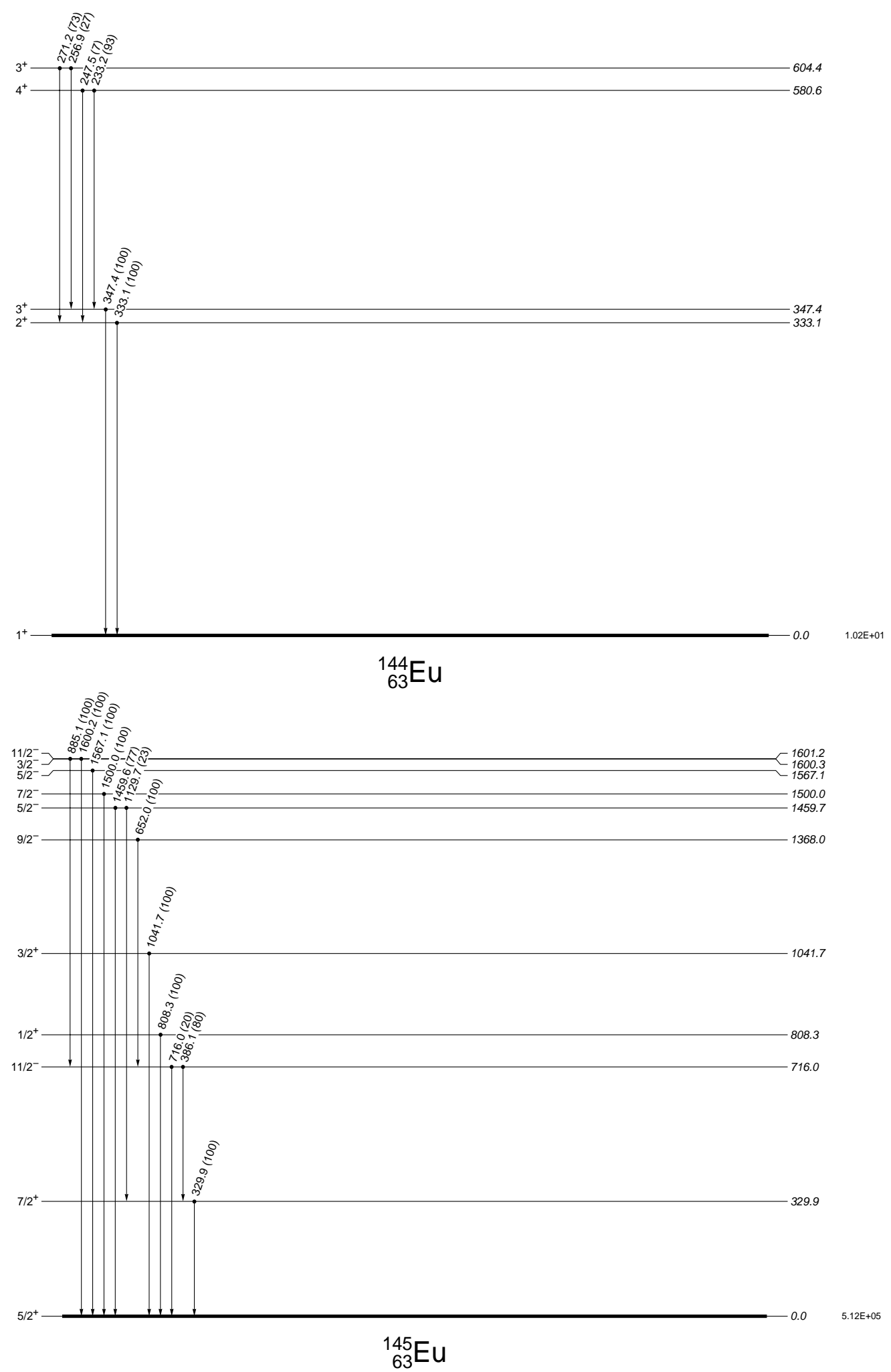

Fig. 20.- (continued) 

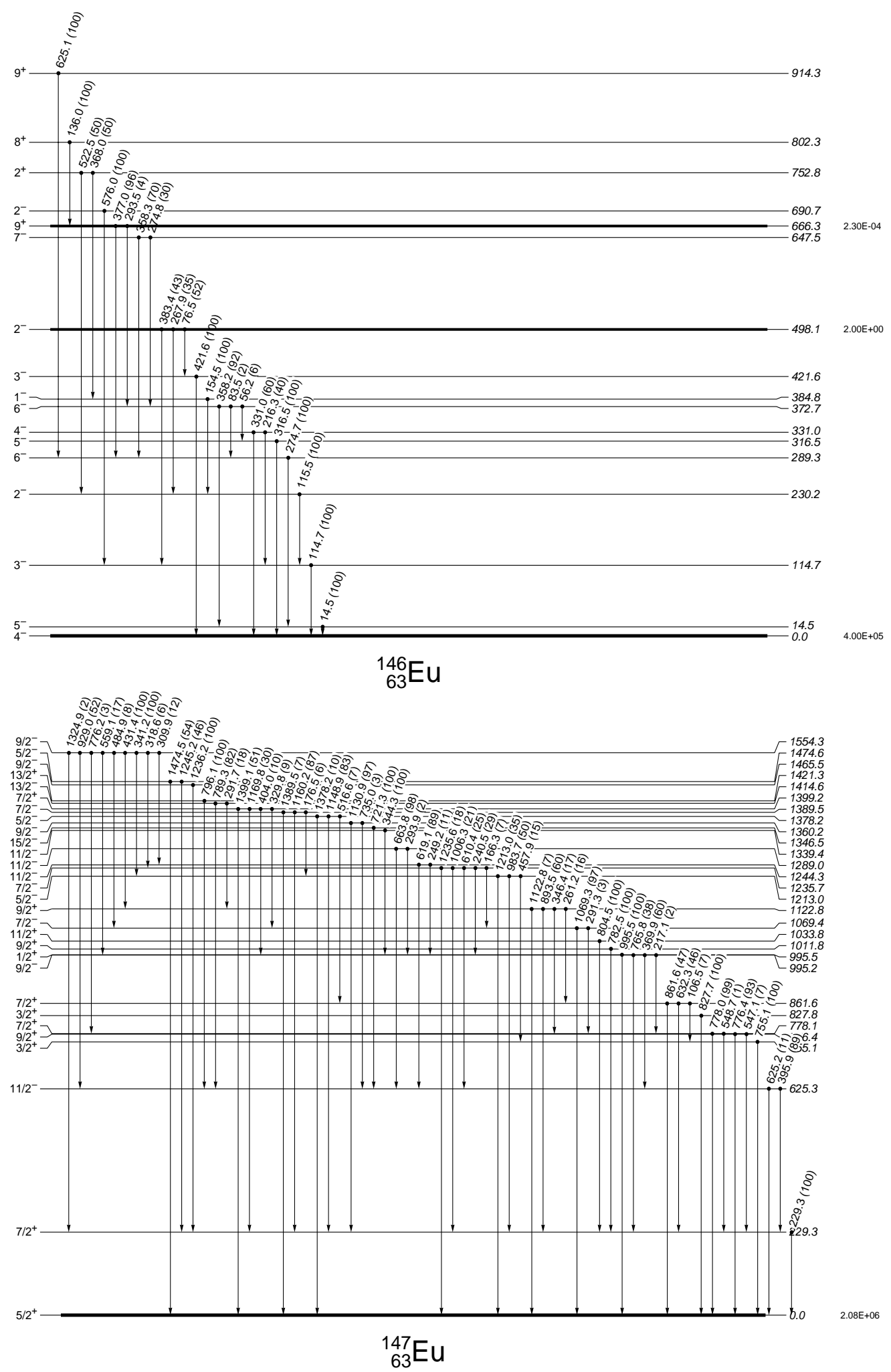

Fig. 20.- (continued) 

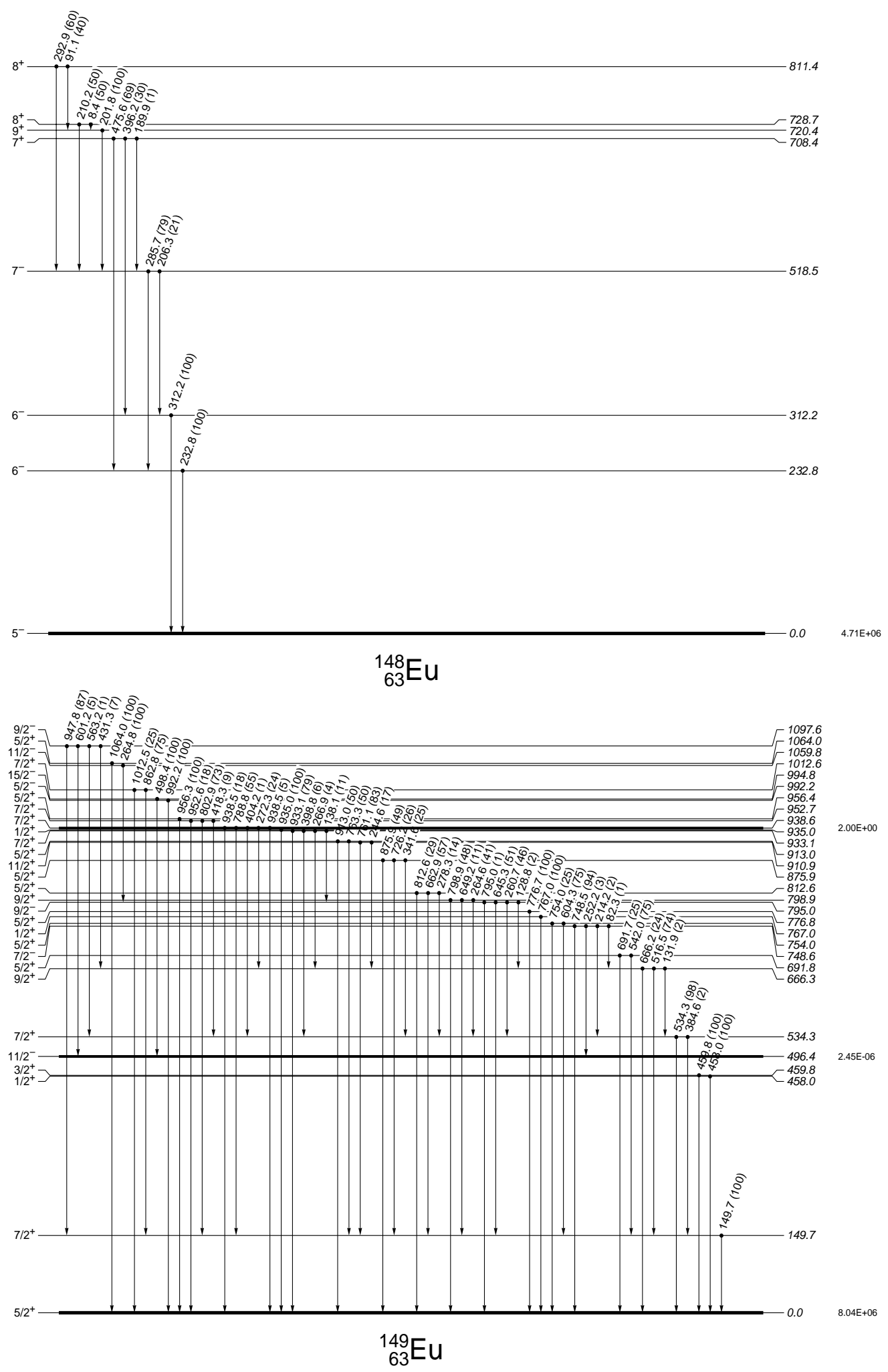

Fig. 20.- (continued) 

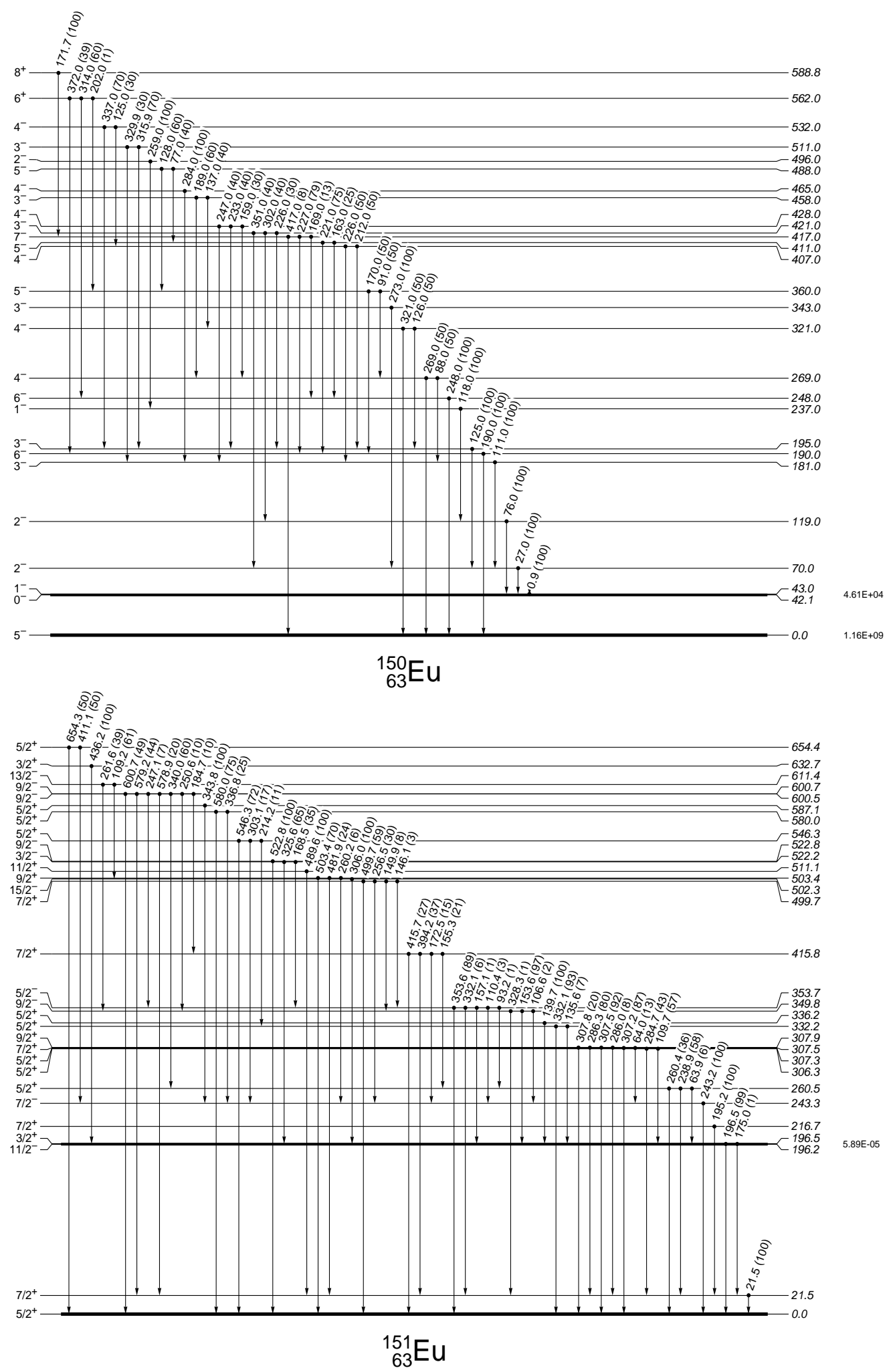

Fig. 20.- (continued) 

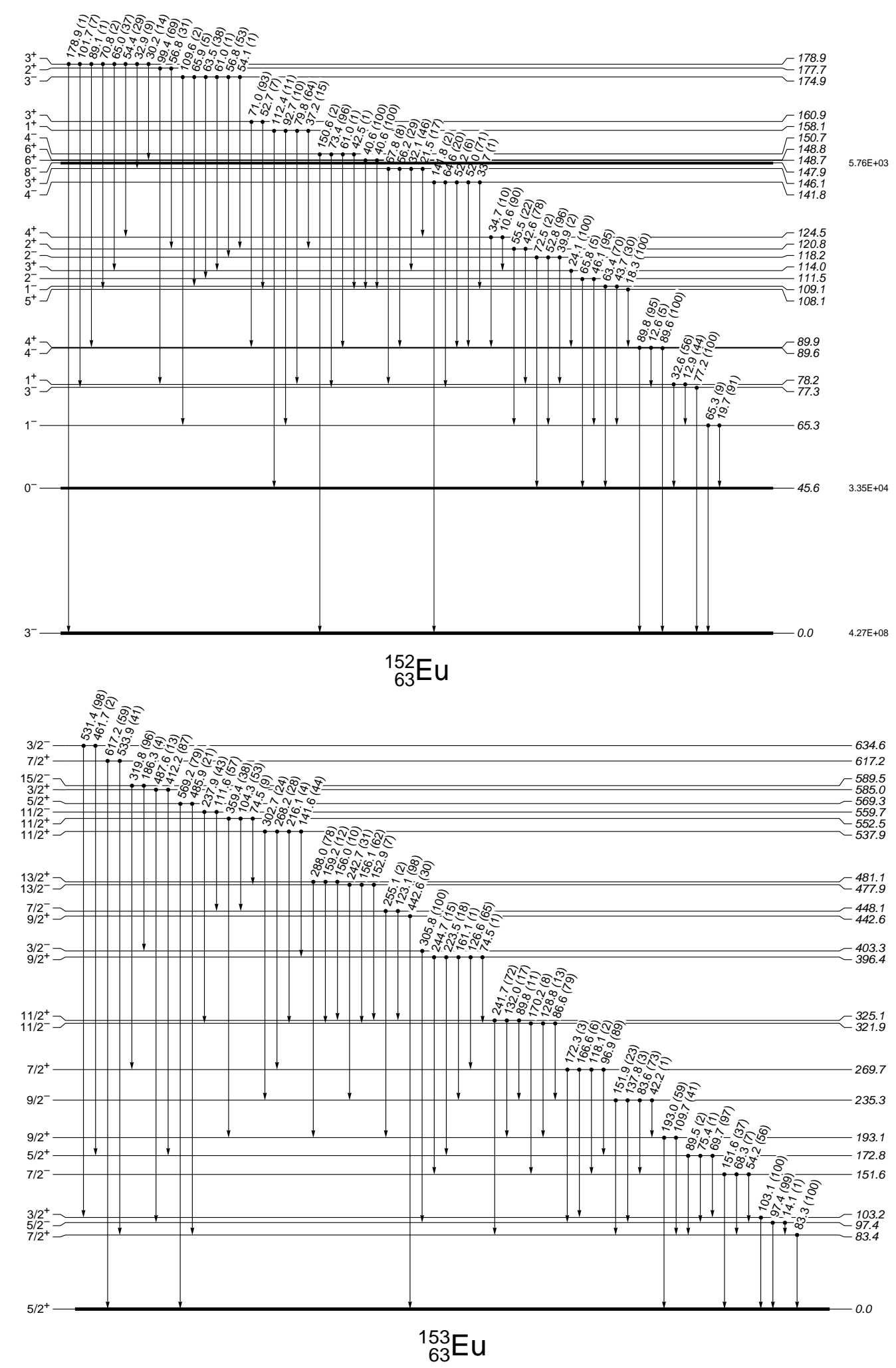

Fig. 20.- (continued) 

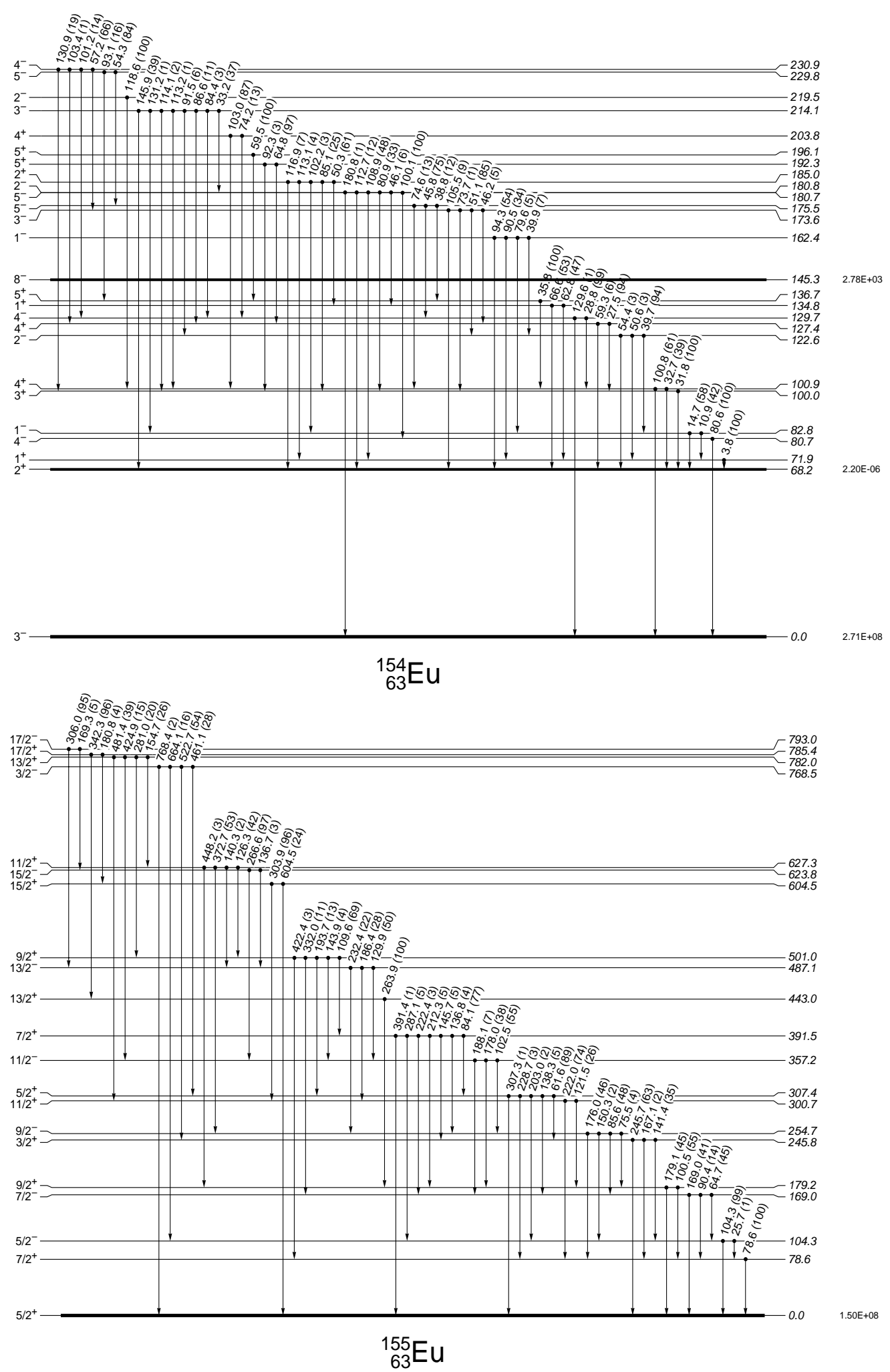

Fig. 20.- (continued) 



Fig. 20.- (continued) 



Fig. 20.- (continued) 


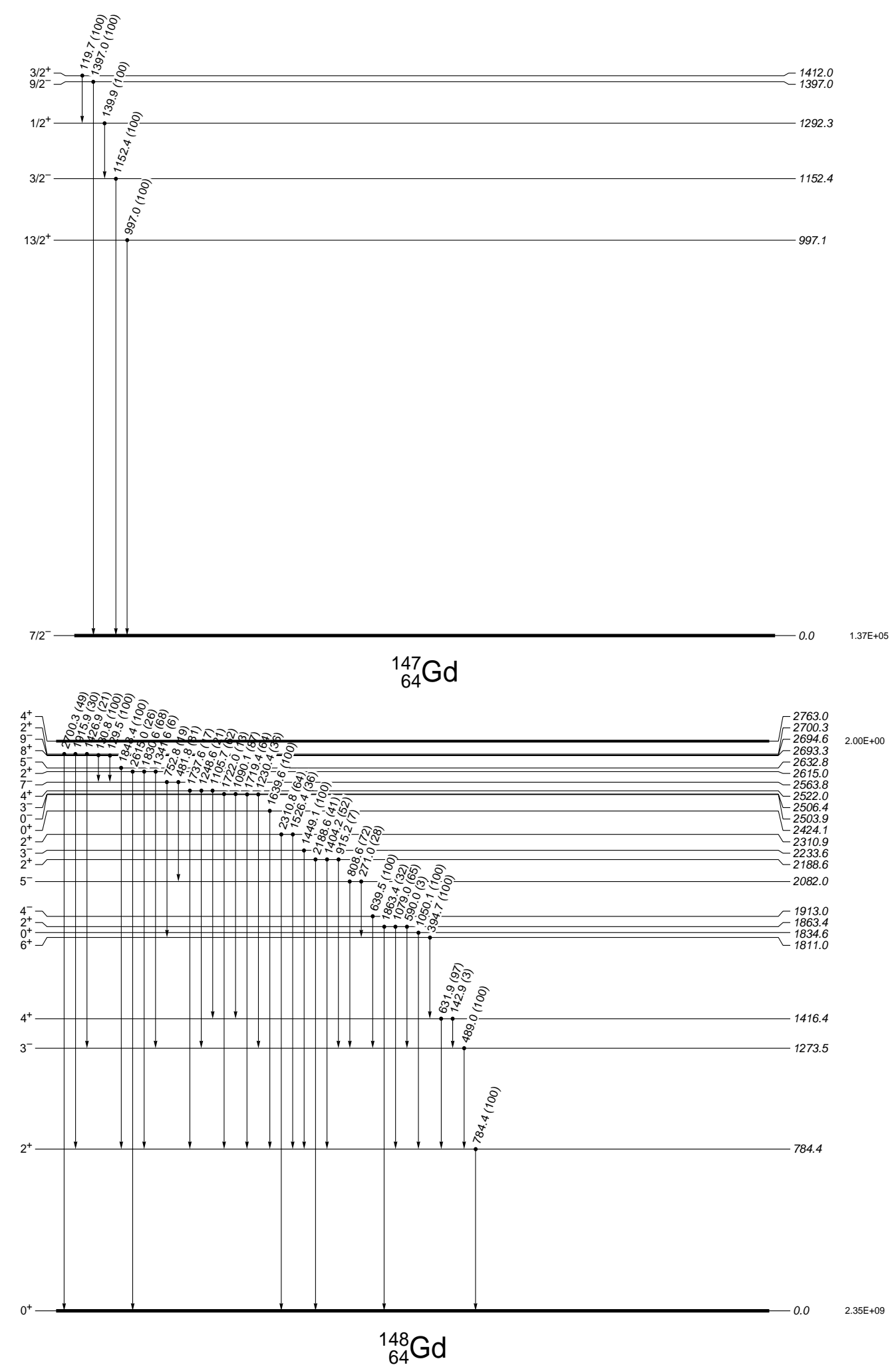

Fig. 20.- (continued) 


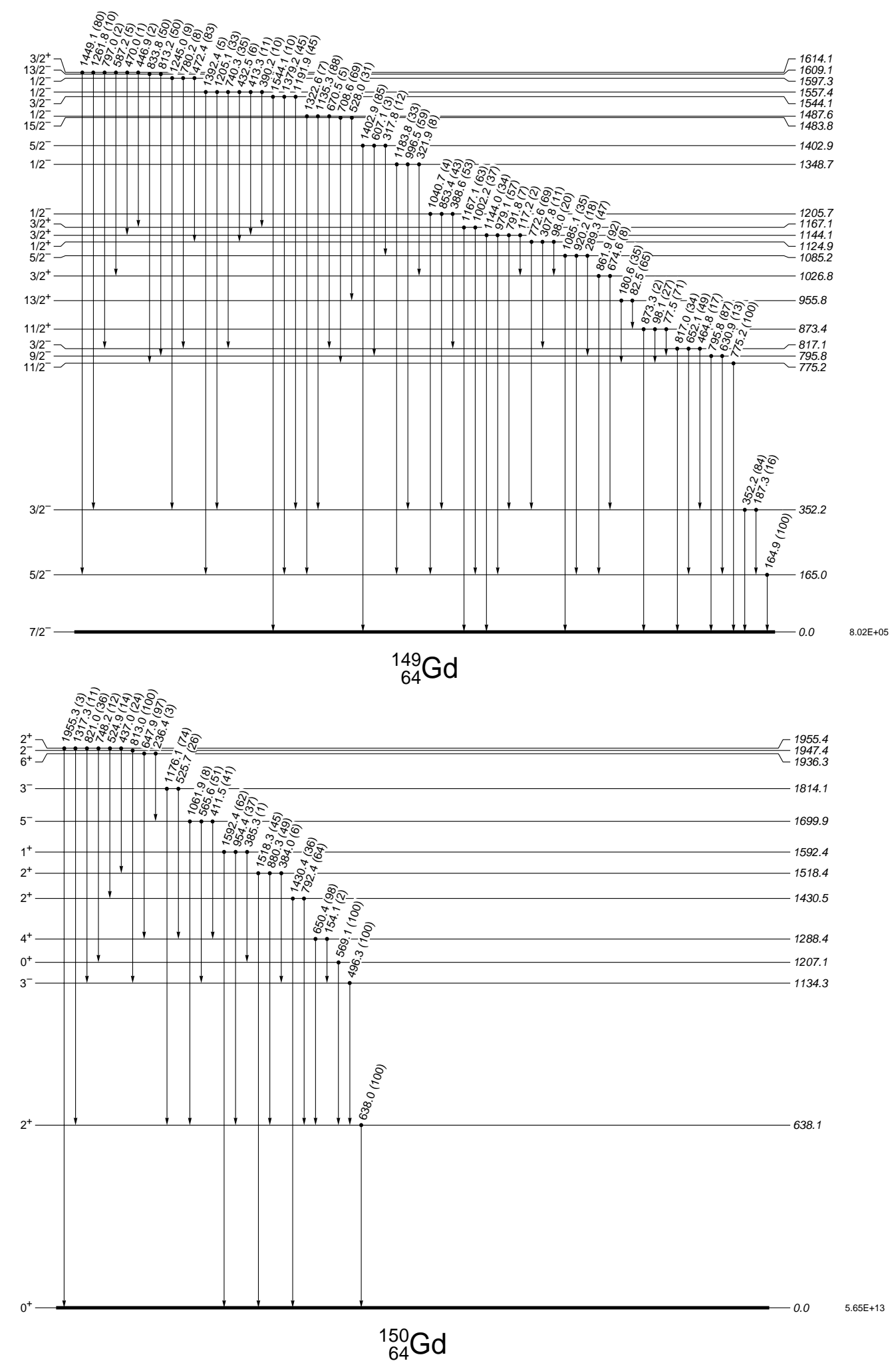

Fig. 20.- (continued) 

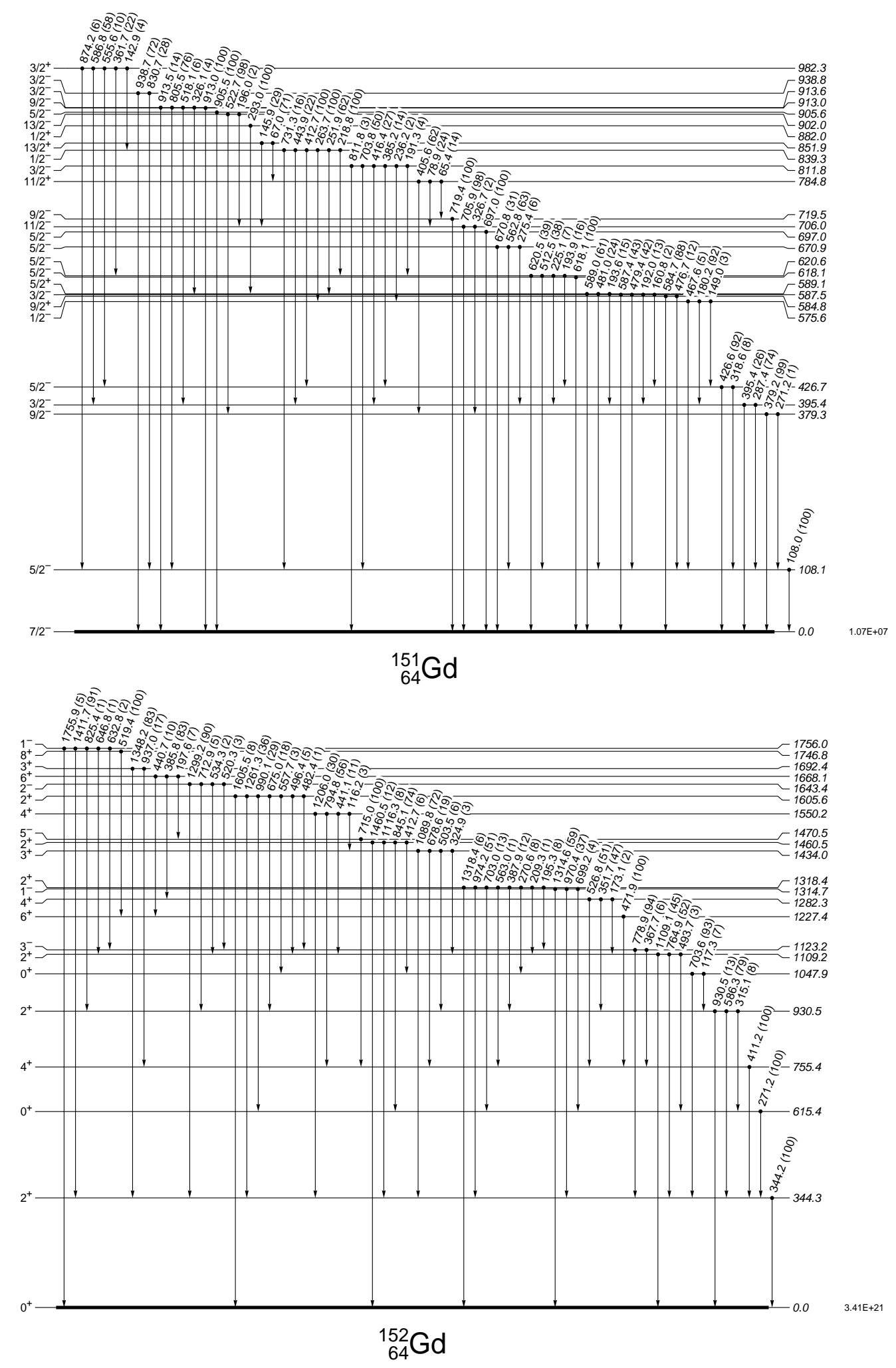

Fig. 20.- (continued) 


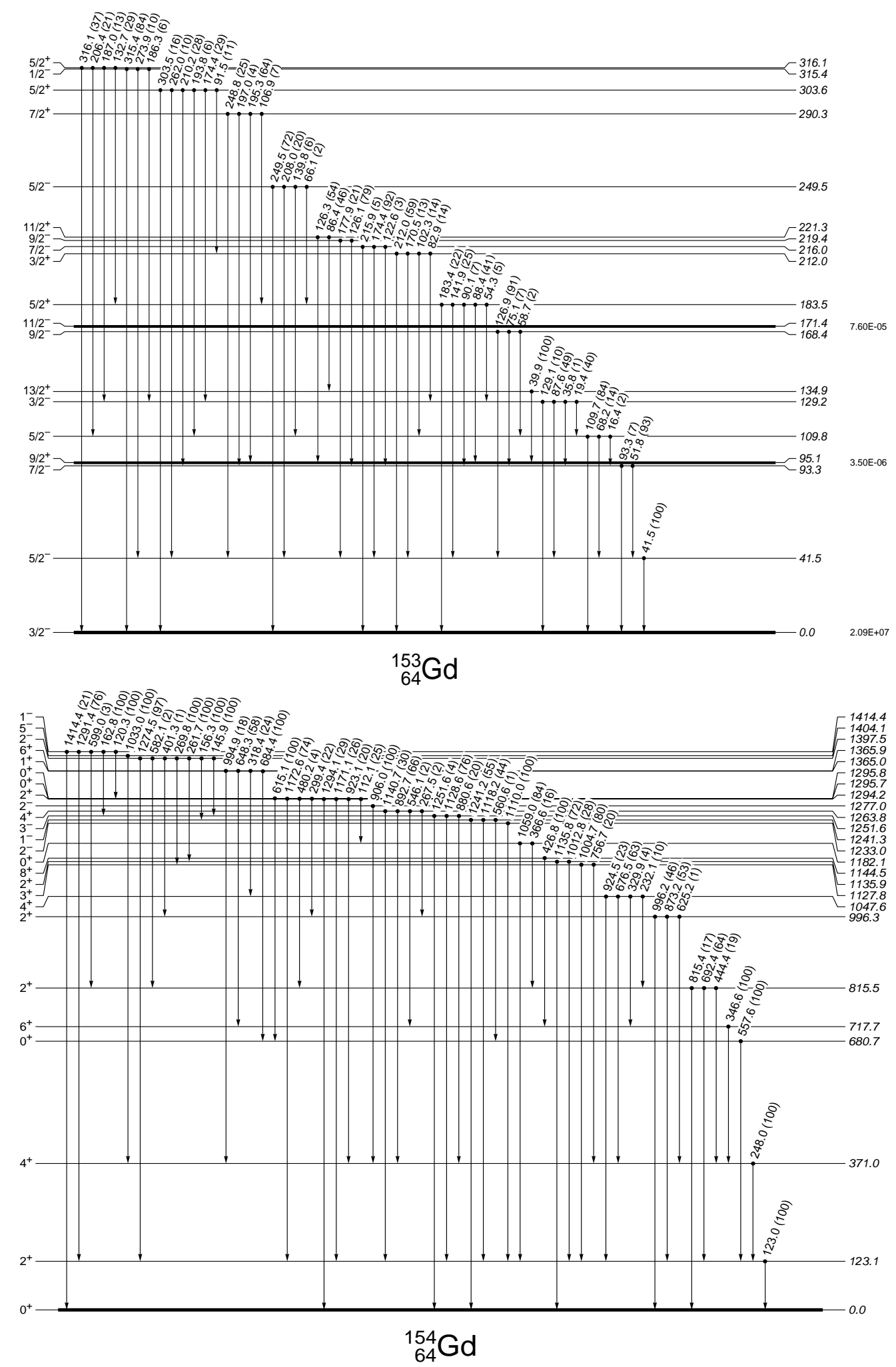

Fig. 20.- (continued) 

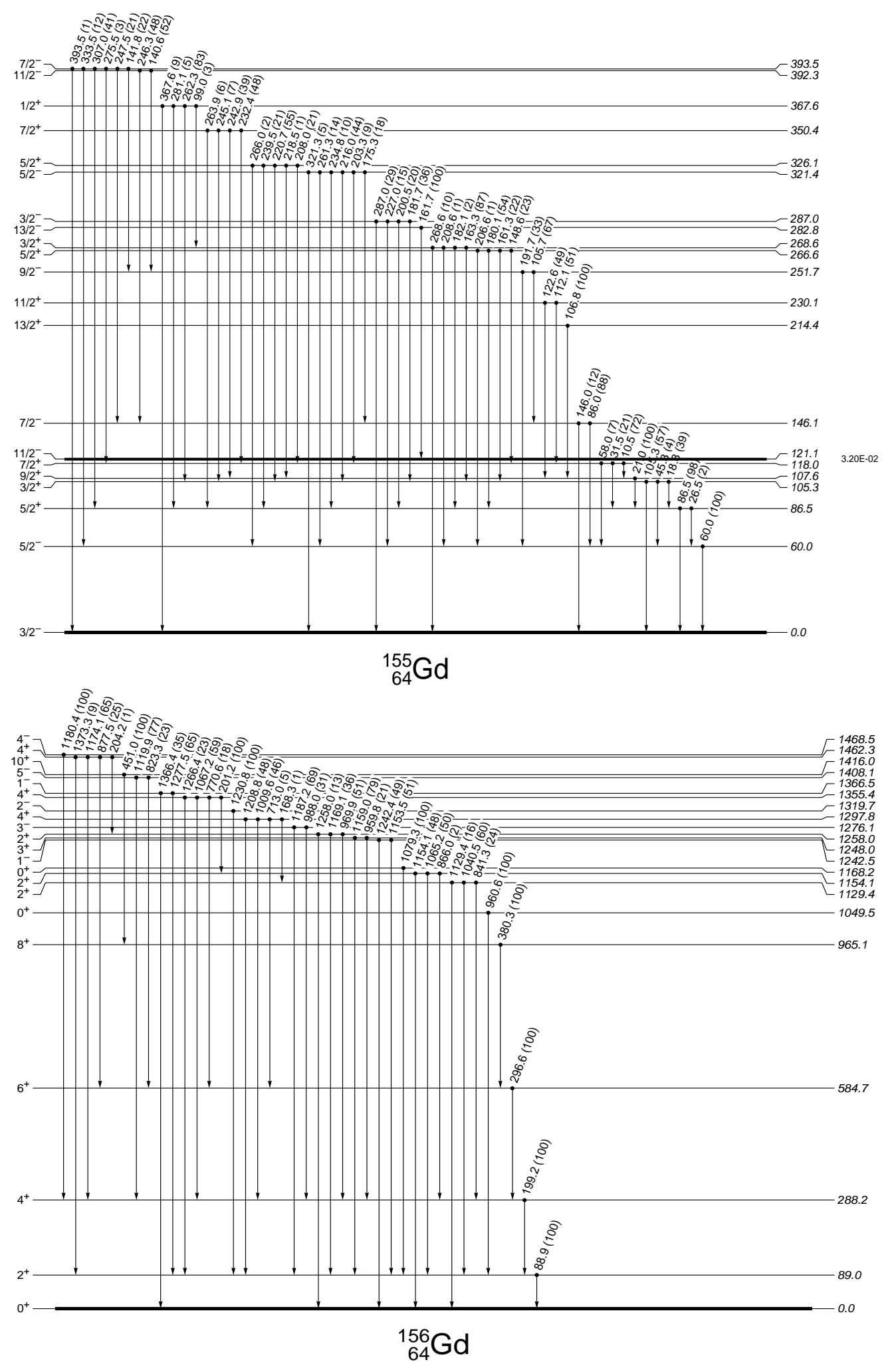

Fig. 20.- (continued) 

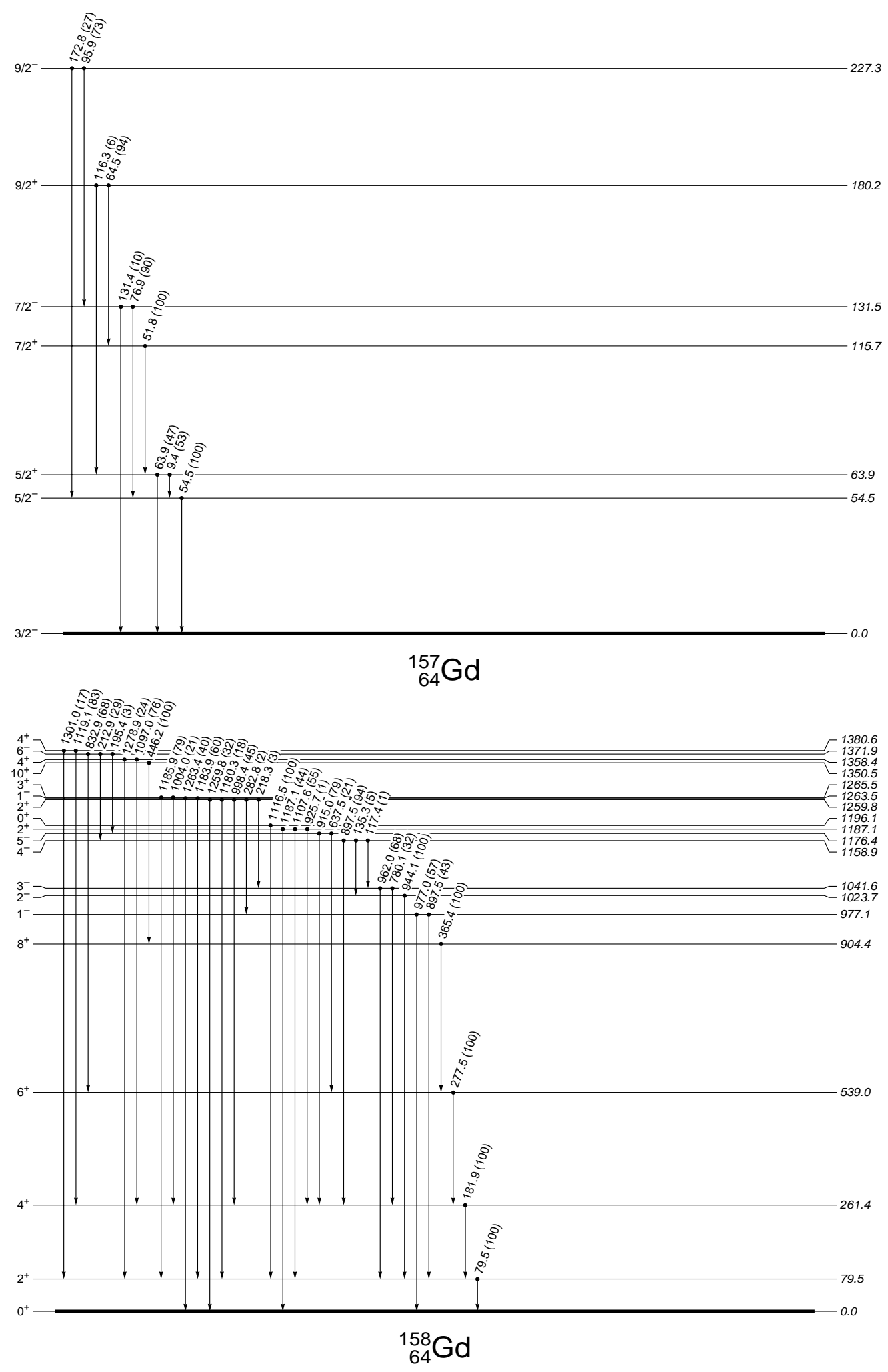

Fig. 20.- (continued) 


\section{A.5. Nuclear Level Density Parameters}

Table 12:: Level density parameters

\begin{tabular}{|c|c|c|c|c|c|c|c|c|c|}
\hline${ }^{A} Z$ & $\begin{array}{r}\tilde{a}(A) \\
(\mathrm{MeV})^{-1}\end{array}$ & $\begin{array}{c}\Delta \\
(\mathrm{MeV})\end{array}$ & $\begin{array}{c}\delta W \\
(\mathrm{MeV})\end{array}$ & $\mathrm{x} / \mathrm{s}$ & $\overline{\sigma^{2}}\left(E_{x}\right)$ & $\begin{array}{c}E_{x} \\
(\mathrm{MeV})\end{array}$ & $\begin{array}{c}E_{0} \\
(\mathrm{MeV})\end{array}$ & $\begin{array}{c}T \\
(\mathrm{MeV})\end{array}$ & $\mathrm{N}$ \\
\hline${ }^{136} \mathrm{Ce}$ & 16.445 & 1.320 & -9.790 & $\mathrm{~s}$ & 6.861 & 8.882 & -2.448 & 0.929 & 6 \\
\hline${ }^{137} \mathrm{Ce}$ & 16.571 & -0.033 & -8.553 & $\mathrm{~s}$ & 6.436 & 6.099 & -3.419 & 0.851 & 5 \\
\hline${ }^{138} \mathrm{Ce}$ & 16.696 & 1.447 & -7.315 & $\mathrm{~s}$ & 6.232 & 7.155 & -1.931 & 0.805 & 18 \\
\hline${ }^{139} \mathrm{Ce}$ & 16.821 & 0.145 & -6.078 & $\mathrm{~s}$ & 3.864 & 1.000 & -0.665 & 0.497 & 7 \\
\hline${ }^{140} \mathrm{Ce}$ & 16.946 & 1.845 & -4.840 & $\mathrm{~s}$ & 5.409 & 5.411 & -0.491 & 0.644 & 17 \\
\hline${ }^{141} \mathrm{Ce}$ & 17.072 & 0.210 & -3.592 & $\mathrm{x}$ & 3.675 & 1.000 & -0.512 & 0.426 & 5 \\
\hline${ }^{142} \mathrm{Ce}$ & 17.197 & 1.415 & -0.893 & $\mathrm{x}$ & 4.732 & 3.890 & -0.322 & 0.505 & 9 \\
\hline${ }^{143} \mathrm{Ce}$ & 17.322 & -0.102 & -1.641 & $\mathrm{x}$ & 3.920 & 1.000 & -0.942 & 0.407 & 0 \\
\hline${ }^{144} \mathrm{Ce}$ & 17.448 & 1.353 & 0.110 & $\mathrm{~s}$ & 4.856 & 4.140 & -0.631 & 0.508 & 3 \\
\hline${ }^{145} \mathrm{Ce}$ & 17.573 & 0.195 & 1.348 & S & 5.105 & 3.729 & -2.391 & 0.537 & 0 \\
\hline${ }^{146} \mathrm{Ce}$ & 17.698 & 1.410 & 2.585 & S & 4.895 & 4.507 & -0.869 & 0.494 & 11 \\
\hline${ }^{147} \mathrm{Ce}$ & 17.824 & 0.068 & 3.585 & $\mathrm{~s}$ & 4.799 & 3.000 & -2.104 & 0.471 & 0 \\
\hline${ }^{148} \mathrm{Ce}$ & 17.949 & 1.165 & 3.064 & $\mathrm{~s}$ & 4.906 & 4.255 & -1.121 & 0.485 & 11 \\
\hline${ }^{149} \mathrm{Ce}$ & 18.074 & 0.207 & 2.544 & $\mathrm{~s}$ & 5.112 & 3.714 & -2.398 & 0.513 & 0 \\
\hline${ }^{150} \mathrm{Ce}$ & 18.200 & 1.268 & 2.023 & $\mathrm{~s}$ & 5.012 & 4.393 & -1.023 & 0.494 & 4 \\
\hline${ }^{151} \mathrm{Ce}$ & 18.325 & 0.270 & 1.503 & $\mathrm{~s}$ & 5.202 & 3.763 & -2.295 & 0.520 & 0 \\
\hline${ }^{152} \mathrm{Ce}$ & 18.451 & 1.245 & 0.982 & $\mathrm{~S}$ & 5.484 & 5.400 & -1.823 & 0.561 & 3 \\
\hline${ }^{137} \mathrm{Pr}$ & 16.571 & -0.070 & -9.790 & $\mathrm{~s}$ & 7.403 & 10.443 & -5.171 & 1.010 & 7 \\
\hline${ }^{138} \mathrm{Pr}$ & 16.696 & -0.910 & -8.553 & $\mathrm{~s}$ & 6.514 & 5.433 & -4.407 & 0.855 & 6 \\
\hline${ }^{139} \mathrm{Pr}$ & 16.821 & 0.045 & -7.315 & $\mathrm{~s}$ & 6.079 & 5.093 & -2.964 & 0.772 & 10 \\
\hline${ }^{140} \mathrm{Pr}$ & 16.946 & -0.820 & -6.078 & $\mathrm{~s}$ & 5.512 & 2.751 & -3.085 & 0.668 & 1 \\
\hline${ }^{141} \mathrm{Pr}$ & 17.072 & 0.445 & -4.840 & $\mathrm{~s}$ & 5.176 & 3.373 & -1.486 & 0.602 & 10 \\
\hline${ }^{142} \mathrm{Pr}$ & 17.197 & -0.705 & -3.768 & $\mathrm{x}$ & 5.369 & 2.851 & -3.094 & 0.618 & 1 \\
\hline${ }^{143} \mathrm{Pr}$ & 17.322 & -0.007 & -2.365 & $\mathrm{~s}$ & 5.291 & 3.542 & -2.459 & 0.593 & 1 \\
\hline${ }^{144} \mathrm{Pr}$ & 17.448 & -0.895 & -1.127 & $\mathrm{~s}$ & 4.570 & 1.165 & -2.341 & 0.473 & 4 \\
\hline${ }^{145} \mathrm{Pr}$ & 17.573 & -0.082 & 0.110 & $\mathrm{~s}$ & 4.339 & 1.666 & -1.327 & 0.431 & 5 \\
\hline${ }^{146} \mathrm{Pr}$ & 17.698 & -0.683 & 1.348 & $\mathrm{~S}$ & 5.123 & 2.845 & -3.264 & 0.535 & 0 \\
\hline${ }^{147} \mathrm{Pr}$ & 17.824 & -0.125 & 2.585 & $\mathrm{~S}$ & 5.075 & 3.395 & -2.741 & 0.518 & 0 \\
\hline${ }^{148} \mathrm{Pr}$ & 17.949 & -0.805 & 3.585 & $\mathrm{~s}$ & 5.042 & 2.708 & -3.446 & 0.505 & 0 \\
\hline${ }^{149} \mathrm{Pr}$ & 18.074 & -0.040 & 3.064 & $\mathrm{~s}$ & 5.085 & 3.467 & -2.661 & 0.508 & 0 \\
\hline${ }^{150} \mathrm{Pr}$ & 18.200 & -0.620 & 2.544 & S & 5.129 & 2.880 & -3.221 & 0.511 & 1 \\
\hline${ }^{151} \mathrm{Pr}$ & 18.325 & -0.085 & 2.023 & $\mathrm{~s}$ & 5.174 & 3.408 & -2.666 & 0.514 & 0 \\
\hline${ }^{152} \mathrm{Pr}$ & 18.451 & -0.585 & 1.503 & $\mathrm{~s}$ & 5.219 & 2.902 & -3.145 & 0.517 & 0 \\
\hline${ }^{153} \mathrm{Pr}$ & 18.576 & -0.080 & 0.982 & $\mathrm{~s}$ & 5.265 & 3.400 & -2.619 & 0.520 & 0 \\
\hline${ }^{138} \mathrm{Nd}$ & 16.696 & 1.265 & -9.790 & $\mathrm{~s}$ & 7.149 & 10.039 & -3.057 & 0.958 & 14 \\
\hline${ }^{139} \mathrm{Nd}$ & 16.821 & 0.018 & -8.553 & $\mathrm{~s}$ & 6.702 & 7.074 & -3.846 & 0.878 & 12 \\
\hline${ }^{140} \mathrm{Nd}$ & 16.946 & 1.417 & -7.315 & $\mathrm{~s}$ & 5.970 & 6.011 & -1.337 & 0.748 & 8 \\
\hline${ }^{141} \mathrm{Nd}$ & 17.072 & 0.173 & -6.078 & $\mathrm{~s}$ & 4.593 & 1.837 & -0.987 & 0.541 & 20 \\
\hline${ }^{142} \mathrm{Nd}$ & 17.197 & 1.860 & -4.840 & $\mathrm{~s}$ & 5.474 & 5.482 & -0.513 & 0.641 & 5 \\
\hline${ }^{143} \mathrm{Nd}$ & 17.322 & 0.242 & -2.939 & $\mathrm{x}$ & 3.632 & 1.000 & -0.449 & 0.406 & 5 \\
\hline${ }^{144} \mathrm{Nd}$ & 17.448 & 1.377 & -1.802 & $\mathrm{x}$ & 4.879 & 3.969 & -0.423 & 0.521 & 19 \\
\hline${ }^{145} \mathrm{Nd}$ & 17.573 & -0.027 & -1.531 & $\mathrm{x}$ & 3.877 & 1.000 & -0.822 & 0.395 & 16 \\
\hline${ }^{146} \mathrm{Nd}$ & 17.698 & 1.205 & -0.307 & $\mathrm{x}$ & 5.058 & 4.321 & -1.011 & 0.530 & 15 \\
\hline${ }^{147} \mathrm{Nd}$ & 17.824 & 0.145 & 1.255 & $\mathrm{x}$ & 4.850 & 2.926 & -1.861 & 0.487 & 10 \\
\hline${ }^{148} \mathrm{Nd}$ & 17.949 & 1.225 & 4.204 & $\mathrm{x}$ & 4.662 & 3.863 & -0.725 & 0.444 & 13 \\
\hline${ }^{149} \mathrm{Nd}$ & 18.074 & 0.118 & 3.362 & $\mathrm{x}$ & 5.005 & 3.449 & -2.370 & 0.494 & 21 \\
\hline${ }^{150} \mathrm{Nd}$ & 18.200 & 1.335 & 3.064 & $\mathrm{~s}$ & 5.206 & 5.125 & -1.518 & 0.522 & 11 \\
\hline${ }^{151} \mathrm{Nd}$ & 18.325 & 0.245 & 2.276 & $\mathrm{x}$ & 5.538 & 4.867 & -3.262 & 0.574 & 3 \\
\hline
\end{tabular}


Table 12: (continued)

\begin{tabular}{|c|c|c|c|c|c|c|c|c|c|}
\hline$\overline{{ }^{A} Z}$ & $\begin{array}{r}\tilde{a}(A) \\
(\mathrm{MeV})^{-1}\end{array}$ & $\begin{array}{c}\Delta \\
(\mathrm{MeV})\end{array}$ & $\begin{array}{c}\delta W \\
(\mathrm{MeV})\end{array}$ & $\mathrm{x} / \mathrm{s}$ & $\overline{\overline{\sigma^{2}}\left(E_{x}\right)}$ & $\begin{array}{c}E_{x} \\
(\mathrm{MeV})\end{array}$ & $\begin{array}{c}E_{0} \\
(\mathrm{MeV})\end{array}$ & $\begin{array}{c}T \\
(\mathrm{MeV})\end{array}$ & $\mathrm{N}$ \\
\hline${ }^{152} \mathrm{Nd}$ & 18.451 & 1.325 & 2.023 & $\mathrm{~s}$ & 5.252 & 4.978 & -1.385 & 0.521 & 4 \\
\hline${ }^{153} \mathrm{Nd}$ & 18.576 & 0.240 & 1.503 & $\mathrm{~s}$ & 5.236 & 3.720 & -2.316 & 0.515 & 0 \\
\hline${ }^{154} \mathrm{Nd}$ & 18.702 & 1.317 & 0.982 & $\mathrm{~s}$ & 5.810 & 6.391 & -2.492 & 0.604 & 2 \\
\hline${ }^{139} \mathrm{Pm}$ & 16.821 & -0.113 & -9.790 & $\mathrm{~s}$ & 7.488 & 10.550 & -5.293 & 1.005 & 18 \\
\hline${ }^{140} \mathrm{Pm}$ & 16.946 & -0.935 & -8.553 & $\mathrm{~s}$ & 5.744 & 2.636 & -3.017 & 0.724 & 1 \\
\hline${ }^{141} \mathrm{Pm}$ & 17.072 & -0.002 & -7.315 & $\mathrm{~s}$ & 6.222 & 5.375 & -3.202 & 0.780 & 17 \\
\hline${ }^{142} \mathrm{Pm}$ & 17.197 & -0.832 & -6.078 & $\mathrm{~s}$ & 4.825 & 1.169 & -2.175 & 0.562 & 10 \\
\hline${ }^{143} \mathrm{Pm}$ & 17.322 & 0.440 & -4.840 & $\mathrm{~s}$ & 4.922 & 2.749 & -1.106 & 0.555 & 26 \\
\hline${ }^{144} \mathrm{Pm}$ & 17.448 & -0.690 & -3.603 & S & 5.450 & 3.000 & -3.179 & 0.618 & 10 \\
\hline${ }^{145} \mathrm{Pm}$ & 17.573 & -0.037 & -2.365 & S & 4.502 & 1.755 & -1.294 & 0.469 & 18 \\
\hline${ }^{146} \mathrm{Pm}$ & 17.698 & -0.970 & -1.127 & $\mathrm{~S}$ & 5.266 & 2.557 & -3.461 & 0.566 & 0 \\
\hline${ }^{147} \mathrm{Pm}$ & 17.824 & -0.090 & 0.110 & $\mathrm{~s}$ & 4.573 & 2.000 & -1.571 & 0.453 & 1 \\
\hline${ }^{148} \mathrm{Pm}$ & 17.949 & -0.648 & 1.442 & $\mathrm{x}$ & 3.372 & 0.000 & -1.218 & 0.320 & 5 \\
\hline${ }^{149} \mathrm{Pm}$ & 18.074 & 0.005 & 2.585 & $\mathrm{~s}$ & 4.930 & 3.048 & -2.233 & 0.485 & 17 \\
\hline${ }^{150} \mathrm{Pm}$ & 18.200 & -0.683 & 3.585 & $\mathrm{~s}$ & 5.077 & 2.817 & -3.314 & 0.500 & 0 \\
\hline${ }^{151} \mathrm{Pm}$ & 18.325 & 0.035 & 3.064 & $\mathrm{~s}$ & 4.912 & 3.000 & -2.154 & 0.471 & 17 \\
\hline${ }^{152} \mathrm{Pm}$ & 18.451 & -0.600 & 2.544 & $\mathrm{~s}$ & 3.319 & 0.000 & -1.130 & 0.297 & 9 \\
\hline${ }^{153} \mathrm{Pm}$ & 18.576 & 0.010 & 2.023 & $\mathrm{~S}$ & 5.208 & 3.490 & -2.563 & 0.509 & 1 \\
\hline${ }^{154} \mathrm{Pm}$ & 18.702 & -0.545 & 1.503 & $\mathrm{~s}$ & 4.887 & 2.059 & -2.425 & 0.458 & 13 \\
\hline${ }^{155} \mathrm{Pm}$ & 18.827 & 0.020 & 0.982 & $\mathrm{~s}$ & 3.862 & 1.000 & -0.721 & 0.339 & 14 \\
\hline${ }^{156} \mathrm{Pm}$ & 18.953 & -0.562 & 0.462 & $\mathrm{~s}$ & 5.347 & 2.899 & -3.072 & 0.519 & 0 \\
\hline${ }^{140} \mathrm{Sm}$ & 16.946 & 1.285 & -9.790 & $\mathrm{~s}$ & 6.960 & 8.770 & -2.465 & 0.911 & 14 \\
\hline${ }^{141} \mathrm{Sm}$ & 17.072 & 0.015 & -8.553 & $\mathrm{~s}$ & 6.898 & 7.738 & -4.196 & 0.893 & 6 \\
\hline${ }^{142} \mathrm{Sm}$ & 17.197 & 1.397 & -7.315 & $\mathrm{~s}$ & 5.848 & 5.467 & -1.064 & 0.715 & 2 \\
\hline${ }^{143} \mathrm{Sm}$ & 17.322 & 0.145 & -6.078 & $\mathrm{~s}$ & 3.927 & 1.000 & -0.649 & 0.483 & 13 \\
\hline${ }^{144} \mathrm{Sm}$ & 17.448 & 1.882 & -4.840 & $\mathrm{~s}$ & 5.362 & 5.101 & -0.234 & 0.611 & 2 \\
\hline${ }^{145} \mathrm{Sm}$ & 17.573 & 0.270 & -4.211 & $\mathrm{x}$ & 3.695 & 1.000 & -0.434 & 0.426 & 21 \\
\hline${ }^{146} \mathrm{Sm}$ & 17.698 & 1.367 & -2.365 & $\mathrm{~s}$ & 5.015 & 4.093 & -0.512 & 0.533 & 27 \\
\hline${ }^{147} \mathrm{Sm}$ & 17.824 & -0.015 & -1.127 & $\mathrm{~S}$ & 2.583 & 0.118 & 0.000 & 0.000 & 7 \\
\hline${ }^{148} \mathrm{Sm}$ & 17.949 & 1.212 & 0.338 & $\mathrm{x}$ & 4.632 & 3.401 & -0.342 & 0.455 & 4 \\
\hline${ }^{149} \mathrm{Sm}$ & 18.074 & 0.073 & 1.217 & $\mathrm{x}$ & 4.717 & 2.482 & -1.655 & 0.458 & 22 \\
\hline${ }^{150} \mathrm{Sm}$ & 18.200 & 1.242 & 2.513 & $\mathrm{x}$ & 4.877 & 4.104 & -0.853 & 0.472 & 32 \\
\hline${ }^{151} \mathrm{Sm}$ & 18.325 & -0.012 & 3.379 & $\mathrm{x}$ & 5.235 & 3.850 & -2.937 & 0.521 & 21 \\
\hline${ }^{152} \mathrm{Sm}$ & 18.451 & 1.290 & 3.477 & $\mathrm{x}$ & 5.062 & 4.633 & -1.213 & 0.488 & 32 \\
\hline${ }^{153} \mathrm{Sm}$ & 18.576 & 0.182 & 2.729 & $\mathrm{x}$ & 5.539 & 4.752 & -3.307 & 0.562 & 10 \\
\hline${ }^{154} \mathrm{Sm}$ & 18.702 & 1.260 & 2.023 & $\mathrm{~S}$ & 5.177 & 4.608 & -1.209 & 0.499 & 7 \\
\hline${ }^{155} \mathrm{Sm}$ & 18.827 & 0.198 & 0.767 & $\mathrm{x}$ & 5.559 & 4.352 & -2.865 & 0.557 & 6 \\
\hline${ }^{156} \mathrm{Sm}$ & 18.953 & 1.290 & 0.982 & $\mathrm{~s}$ & 5.434 & 5.066 & -1.484 & 0.531 & 7 \\
\hline${ }^{157} \mathrm{Sm}$ & 19.079 & 0.207 & 0.462 & $\mathrm{~s}$ & 5.365 & 3.663 & -2.299 & 0.517 & 1 \\
\hline${ }^{158} \mathrm{Sm}$ & 19.204 & 1.192 & -0.059 & $\mathrm{~s}$ & 5.929 & 6.156 & -2.467 & 0.601 & 2 \\
\hline${ }^{159} \mathrm{Sm}$ & 19.330 & 0.248 & -0.580 & $\mathrm{~s}$ & 5.463 & 3.691 & -2.214 & 0.524 & 0 \\
\hline${ }^{141} \mathrm{Eu}$ & 17.072 & -0.143 & -9.790 & $\mathrm{~s}$ & 7.488 & 10.122 & -5.154 & 0.987 & 14 \\
\hline${ }^{142} \mathrm{Eu}$ & 17.197 & -0.910 & -8.553 & $\mathrm{~s}$ & 5.785 & 2.646 & -2.986 & 0.717 & 1 \\
\hline${ }^{143} \mathrm{Eu}$ & 17.322 & 0.002 & -7.315 & $\mathrm{~s}$ & 6.413 & 5.914 & -3.504 & 0.796 & 4 \\
\hline${ }^{144} \mathrm{Eu}$ & 17.448 & -0.902 & -6.078 & $\mathrm{~s}$ & 3.996 & 0.000 & -1.707 & 0.482 & 4 \\
\hline${ }^{145} \mathrm{Eu}$ & 17.573 & 0.473 & -4.840 & $\mathrm{~s}$ & 4.979 & 2.814 & -1.093 & 0.552 & 10 \\
\hline${ }^{146} \mathrm{Eu}$ & 17.698 & -0.798 & -3.603 & $\mathrm{~s}$ & 4.599 & 1.000 & -2.046 & 0.487 & 10 \\
\hline${ }^{147} \mathrm{Eu}$ & 17.824 & -0.055 & -2.365 & $\mathrm{~s}$ & 3.976 & 1.000 & -0.867 & 0.406 & 25 \\
\hline${ }^{148} \mathrm{Eu}$ & 17.949 & -0.923 & -1.127 & $\mathrm{~s}$ & 3.799 & 0.000 & -1.653 & 0.375 & 0 \\
\hline${ }^{149} \mathrm{Eu}$ & 18.074 & -0.017 & 0.110 & $\mathrm{~s}$ & 4.697 & 2.237 & -1.616 & 0.460 & 28 \\
\hline
\end{tabular}


Table 12: (continued)

\begin{tabular}{|c|c|c|c|c|c|c|c|c|c|}
\hline${ }^{\prime{ }^{A} Z}$ & $\begin{array}{r}\tilde{a}(A) \\
(\mathrm{MeV})^{-1}\end{array}$ & $\begin{array}{c}\Delta \\
(\mathrm{MeV})\end{array}$ & $\begin{array}{c}\delta W \\
(\mathrm{MeV})\end{array}$ & $\mathrm{x} / \mathrm{s}$ & $\overline{\overline{\sigma^{2}\left(E_{x}\right)}}$ & $\begin{array}{c}E_{x} \\
(\mathrm{MeV})\end{array}$ & $\begin{array}{c}E_{0} \\
(\mathrm{MeV})\end{array}$ & $\begin{array}{c}T \\
(\mathrm{MeV})\end{array}$ & $\mathrm{N}$ \\
\hline${ }^{150} \mathrm{Eu}$ & 18.200 & -0.708 & 1.348 & $\mathrm{~s}$ & 3.479 & 0.000 & -1.303 & 0.322 & 20 \\
\hline${ }^{151} \mathrm{Eu}$ & 18.325 & 0.000 & 2.585 & $\mathrm{~s}$ & 5.022 & 3.176 & -2.345 & 0.489 & 32 \\
\hline${ }^{152} \mathrm{Eu}$ & 18.451 & -0.690 & 4.165 & $\mathrm{x}$ & 5.070 & 2.764 & -3.302 & 0.487 & 41 \\
\hline${ }^{153} \mathrm{Eu}$ & 18.576 & 0.037 & 3.064 & $\mathrm{~s}$ & 5.055 & 3.262 & -2.360 & 0.483 & 36 \\
\hline${ }^{154} \mathrm{Eu}$ & 18.702 & -0.635 & 2.716 & $\mathrm{x}$ & 5.199 & 2.868 & -3.248 & 0.501 & 40 \\
\hline${ }^{155} \mathrm{Eu}$ & 18.827 & 0.025 & 2.023 & $\mathrm{~s}$ & 4.604 & 2.094 & -1.463 & 0.414 & 31 \\
\hline${ }^{156} \mathrm{Eu}$ & 18.953 & -0.605 & 1.503 & $\mathrm{~S}$ & 4.614 & 1.406 & -2.045 & 0.413 & 30 \\
\hline${ }^{157} \mathrm{Eu}$ & 19.079 & -0.030 & 0.982 & $\mathrm{~s}$ & 4.668 & 2.000 & -1.478 & 0.419 & 1 \\
\hline${ }^{158} \mathrm{Eu}$ & 19.204 & -0.590 & 0.462 & $\mathrm{~s}$ & 5.382 & 2.859 & -3.092 & 0.514 & 0 \\
\hline${ }^{159} \mathrm{Eu}$ & 19.330 & -0.040 & -0.059 & $\mathrm{~s}$ & 5.186 & 2.824 & -2.084 & 0.483 & 4 \\
\hline${ }^{160} \mathrm{Eu}$ & 19.455 & -0.575 & -0.580 & $\mathrm{~s}$ & 5.480 & 2.862 & -3.033 & 0.522 & 0 \\
\hline${ }^{142} \mathrm{Gd}$ & 17.197 & 1.445 & -9.790 & $\mathrm{~S}$ & 7.282 & 10.304 & -2.935 & 0.944 & 4 \\
\hline${ }^{143} \mathrm{Gd}$ & 17.322 & 0.080 & -8.553 & $\mathrm{~s}$ & 7.009 & 8.081 & -4.280 & 0.895 & 5 \\
\hline${ }^{144} \mathrm{Gd}$ & 17.448 & 1.300 & -7.315 & $\mathrm{~s}$ & 5.831 & 5.189 & -1.062 & 0.699 & 10 \\
\hline${ }^{145} \mathrm{Gd}$ & 17.573 & 0.212 & -6.078 & $\mathrm{~s}$ & 3.879 & 1.000 & -0.557 & 0.475 & 6 \\
\hline${ }^{146} \mathrm{Gd}$ & 17.698 & 1.902 & -4.840 & $\mathrm{~s}$ & 4.892 & 4.047 & 0.458 & 0.535 & 5 \\
\hline${ }^{147} \mathrm{Gd}$ & 17.824 & 0.242 & -3.603 & $\mathrm{~s}$ & 3.725 & 1.000 & -0.450 & 0.408 & 5 \\
\hline${ }^{148} \mathrm{Gd}$ & 17.949 & 1.387 & -2.365 & $\mathrm{~s}$ & 3.486 & 2.000 & 0.768 & 0.376 & 22 \\
\hline${ }^{149} \mathrm{Gd}$ & 18.074 & 0.003 & -1.127 & $\mathrm{~s}$ & 3.269 & 0.500 & -0.565 & 0.351 & 6 \\
\hline${ }^{150} \mathrm{Gd}$ & 18.200 & 1.240 & 0.110 & $\mathrm{~s}$ & 4.650 & 3.373 & -0.271 & 0.450 & 12 \\
\hline${ }^{151} \mathrm{Gd}$ & 18.325 & 0.035 & 1.348 & $\mathrm{~s}$ & 4.660 & 2.274 & -1.569 & 0.441 & 25 \\
\hline${ }^{152} \mathrm{Gd}$ & 18.451 & 1.255 & 2.585 & $\mathrm{~s}$ & 4.871 & 4.026 & -0.773 & 0.461 & 29 \\
\hline${ }^{153} \mathrm{Gd}$ & 18.576 & -0.007 & 3.579 & $\mathrm{x}$ & 5.229 & 3.751 & -2.855 & 0.509 & 18 \\
\hline${ }^{154} \mathrm{Gd}$ & 18.702 & 1.247 & 3.064 & $\mathrm{~s}$ & 5.278 & 5.016 & -1.592 & 0.512 & 26 \\
\hline${ }^{155} \mathrm{Gd}$ & 18.827 & 0.103 & 2.946 & $\mathrm{x}$ & 5.293 & 3.840 & -2.710 & 0.510 & 41 \\
\hline${ }^{156} \mathrm{Gd}$ & 18.953 & 1.223 & 1.540 & $\mathrm{x}$ & 5.344 & 4.839 & -1.446 & 0.516 & 20 \\
\hline${ }^{157} \mathrm{Gd}$ & 19.079 & 0.130 & 1.417 & $\mathrm{x}$ & 5.399 & 3.822 & -2.595 & 0.519 & 6 \\
\hline${ }^{158} \mathrm{Gd}$ & 19.204 & 1.190 & 0.712 & $\mathrm{x}$ & 5.495 & 4.980 & -1.586 & 0.531 & 18 \\
\hline${ }^{159} \mathrm{Gd}$ & 19.330 & 0.162 & 0.434 & $\mathrm{x}$ & 5.737 & 4.546 & -3.070 & 0.564 & 8 \\
\hline${ }^{160} \mathrm{Gd}$ & 19.455 & 1.185 & -0.059 & $\mathrm{~s}$ & 5.455 & 4.640 & -1.305 & 0.516 & 4 \\
\hline${ }^{161} \mathrm{Gd}$ & 19.581 & 0.170 & -0.694 & $\mathrm{x}$ & 5.505 & 3.602 & -2.280 & 0.521 & 1 \\
\hline${ }^{143} \mathrm{~Tb}$ & 17.322 & 0.027 & -9.790 & $\mathrm{~s}$ & 5.702 & 3.000 & -1.669 & 0.716 & 3 \\
\hline${ }^{144} \mathrm{~Tb}$ & 17.448 & -0.832 & -8.553 & $\mathrm{~s}$ & 6.152 & 3.628 & -3.376 & 0.754 & 5 \\
\hline${ }^{145} \mathrm{~Tb}$ & 17.573 & -0.032 & -7.315 & $\mathrm{~s}$ & 5.786 & 3.671 & -2.290 & 0.686 & 3 \\
\hline${ }^{146} \mathrm{~Tb}$ & 17.698 & -0.987 & -6.078 & $\mathrm{~s}$ & 5.629 & 2.540 & -3.230 & 0.647 & 0 \\
\hline${ }^{147} \mathrm{~Tb}$ & 17.824 & 0.525 & -4.840 & $\mathrm{~s}$ & 4.925 & 2.696 & -0.935 & 0.535 & 5 \\
\hline${ }^{148} \mathrm{~Tb}$ & 17.949 & -0.932 & -3.603 & $\mathrm{~s}$ & 4.719 & 1.000 & -2.263 & 0.493 & 6 \\
\hline${ }^{149} \mathrm{~Tb}$ & 18.074 & -0.093 & -2.365 & $\mathrm{~s}$ & 5.003 & 2.481 & -1.868 & 0.516 & 17 \\
\hline${ }^{150} \mathrm{~Tb}$ & 18.200 & -0.892 & -1.127 & $\mathrm{~s}$ & 3.797 & 0.000 & -1.604 & 0.368 & 0 \\
\hline${ }^{151} \mathrm{~Tb}$ & 18.325 & -0.030 & 0.110 & $\mathrm{~s}$ & 5.545 & 4.216 & -3.127 & 0.579 & 3 \\
\hline${ }^{152} \mathrm{~Tb}$ & 18.451 & -0.698 & 1.348 & $\mathrm{~s}$ & 3.493 & 0.000 & -1.285 & 0.317 & 0 \\
\hline${ }^{153} \mathrm{~Tb}$ & 18.576 & 0.033 & 2.585 & $\mathrm{~s}$ & 5.028 & 3.129 & -2.251 & 0.480 & 5 \\
\hline${ }^{154} \mathrm{~Tb}$ & 18.702 & -0.728 & 3.585 & $\mathrm{~s}$ & 3.472 & 0.000 & -1.310 & 0.293 & 0 \\
\hline${ }^{155} \mathrm{~Tb}$ & 18.827 & 0.057 & 3.064 & $\mathrm{~s}$ & 4.956 & 2.951 & -2.078 & 0.458 & 20 \\
\hline${ }^{156} \mathrm{~Tb}$ & 18.953 & -0.627 & 2.544 & $\mathrm{~s}$ & 4.763 & 1.758 & -2.359 & 0.429 & 6 \\
\hline${ }^{157} \mathrm{~Tb}$ & 19.079 & 0.015 & 2.023 & $\mathrm{~s}$ & 4.901 & 2.592 & -1.856 & 0.445 & 20 \\
\hline${ }^{158} \mathrm{~Tb}$ & 19.204 & -0.625 & 1.503 & $\mathrm{~s}$ & 4.674 & 1.431 & -2.098 & 0.413 & 5 \\
\hline${ }^{159} \mathrm{~Tb}$ & 19.330 & 0.010 & 0.982 & $\mathrm{~s}$ & 5.006 & 2.616 & -1.864 & 0.454 & 10 \\
\hline${ }^{160} \mathrm{~Tb}$ & 19.455 & -0.643 & 0.005 & $\mathrm{x}$ & 5.306 & 2.460 & -2.868 & 0.495 & 8 \\
\hline${ }^{161} \mathrm{~Tb}$ & 19.581 & 0.000 & -0.059 & $\mathrm{~s}$ & 5.095 & 2.591 & -1.843 & 0.462 & 8 \\
\hline
\end{tabular}


B. Modeled Cross Sections vs. Experiment 

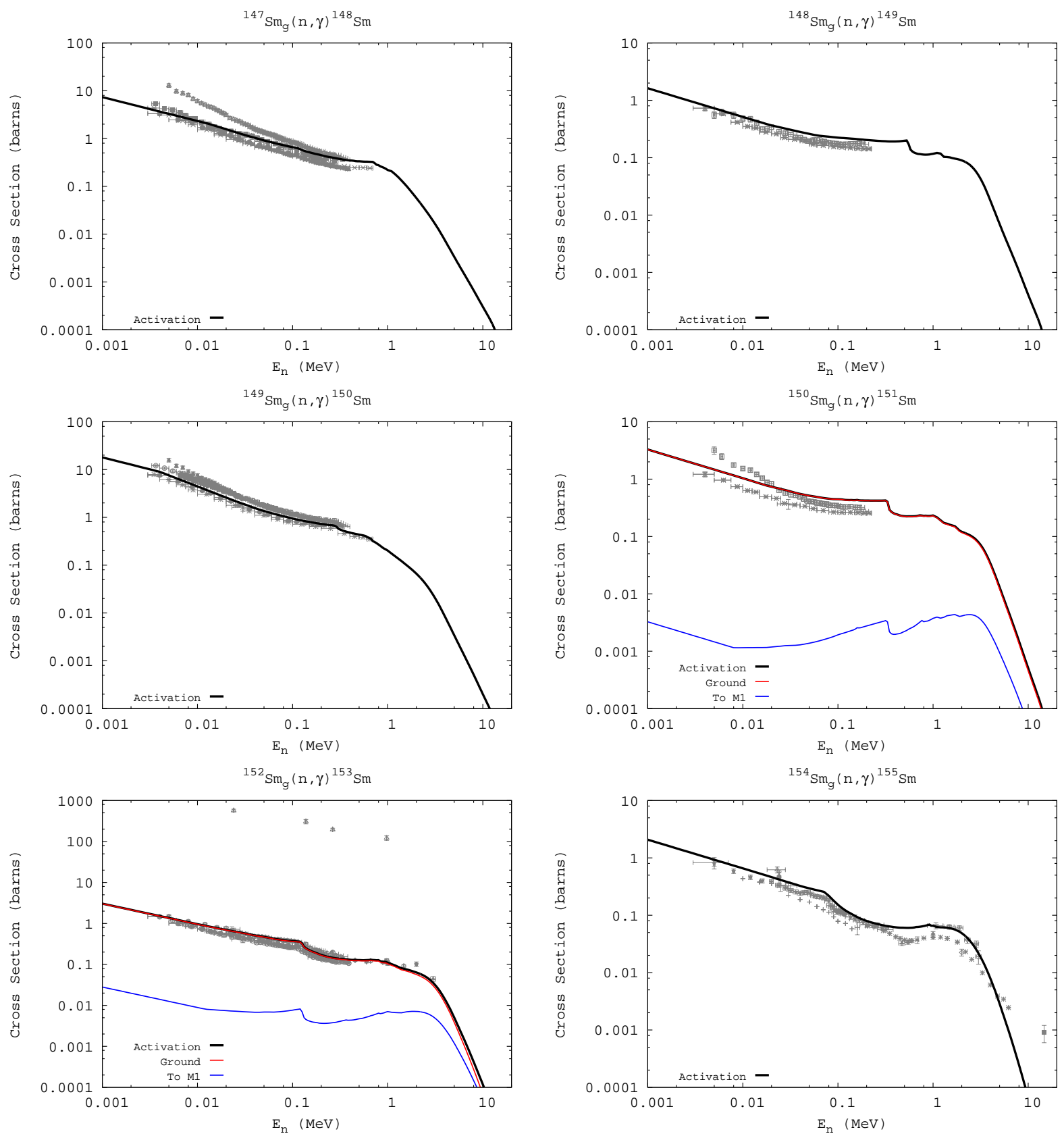

Fig. 21.- Measured vs. calculated $(\mathrm{n}, \gamma)$ cross sections on select targets. 

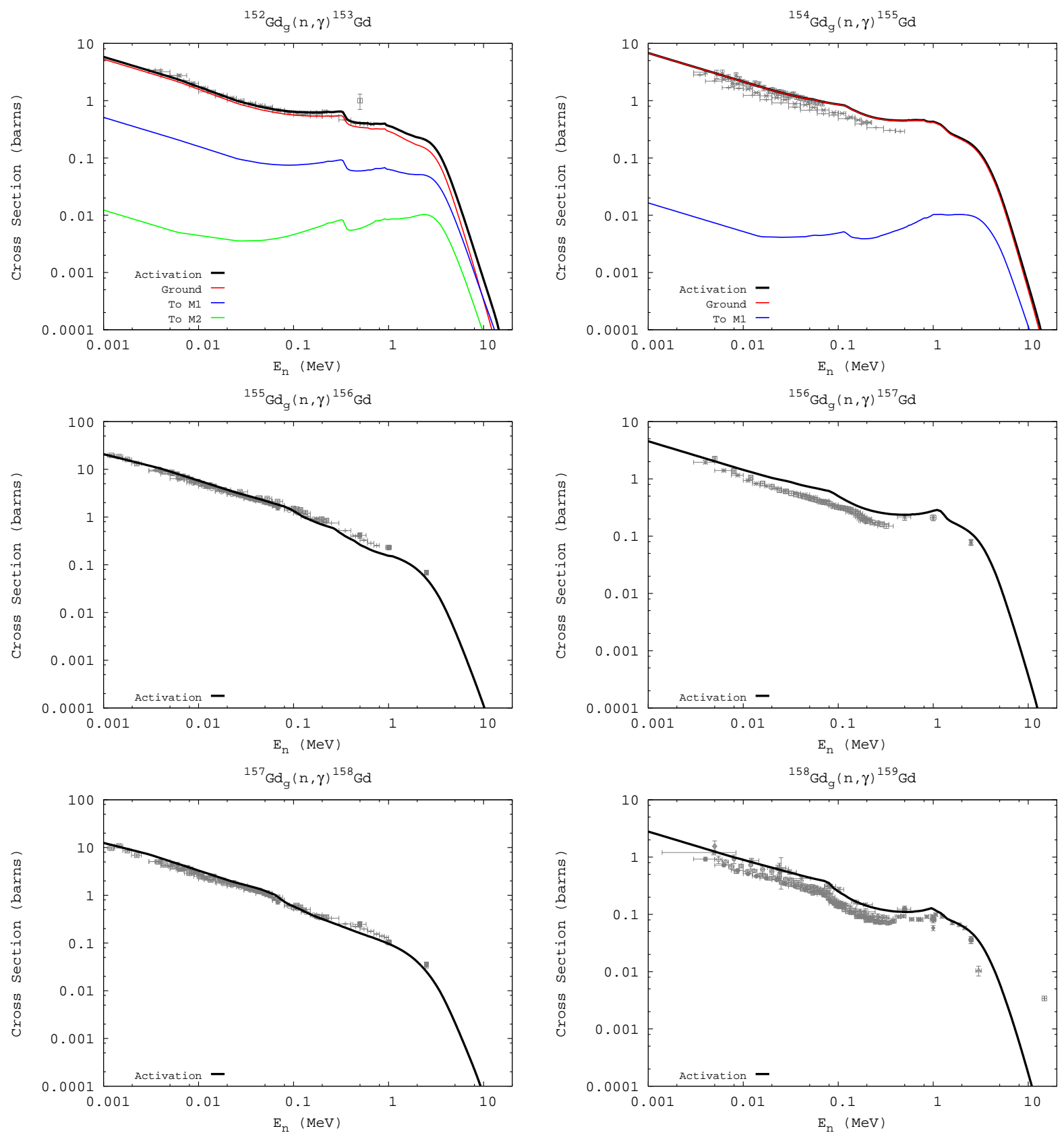

Fig. 21.- (continued) 

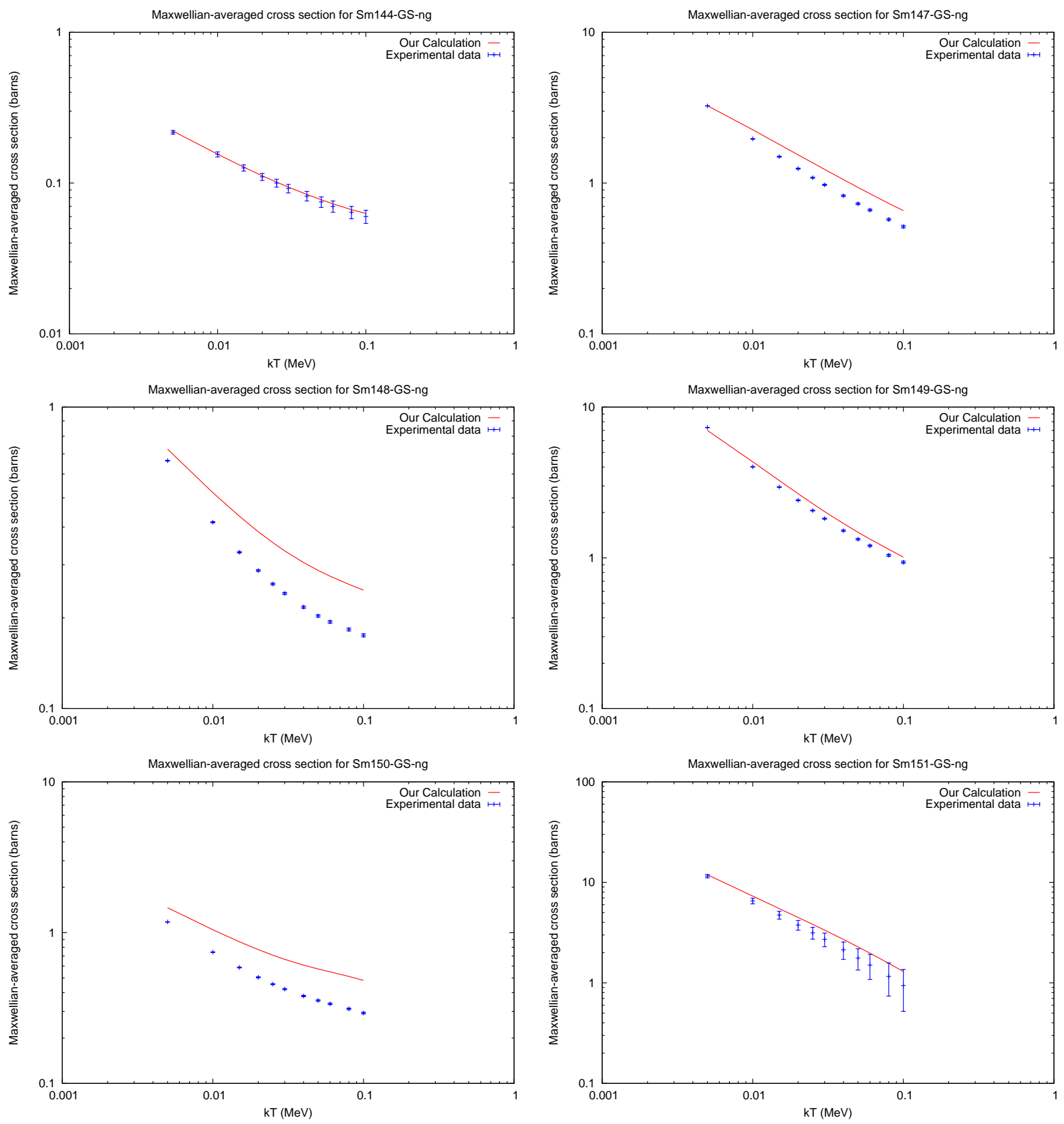

Fig. 22.- Measured vs. calculated Maxwellian averaged (n, $\gamma$ ) cross sections. 

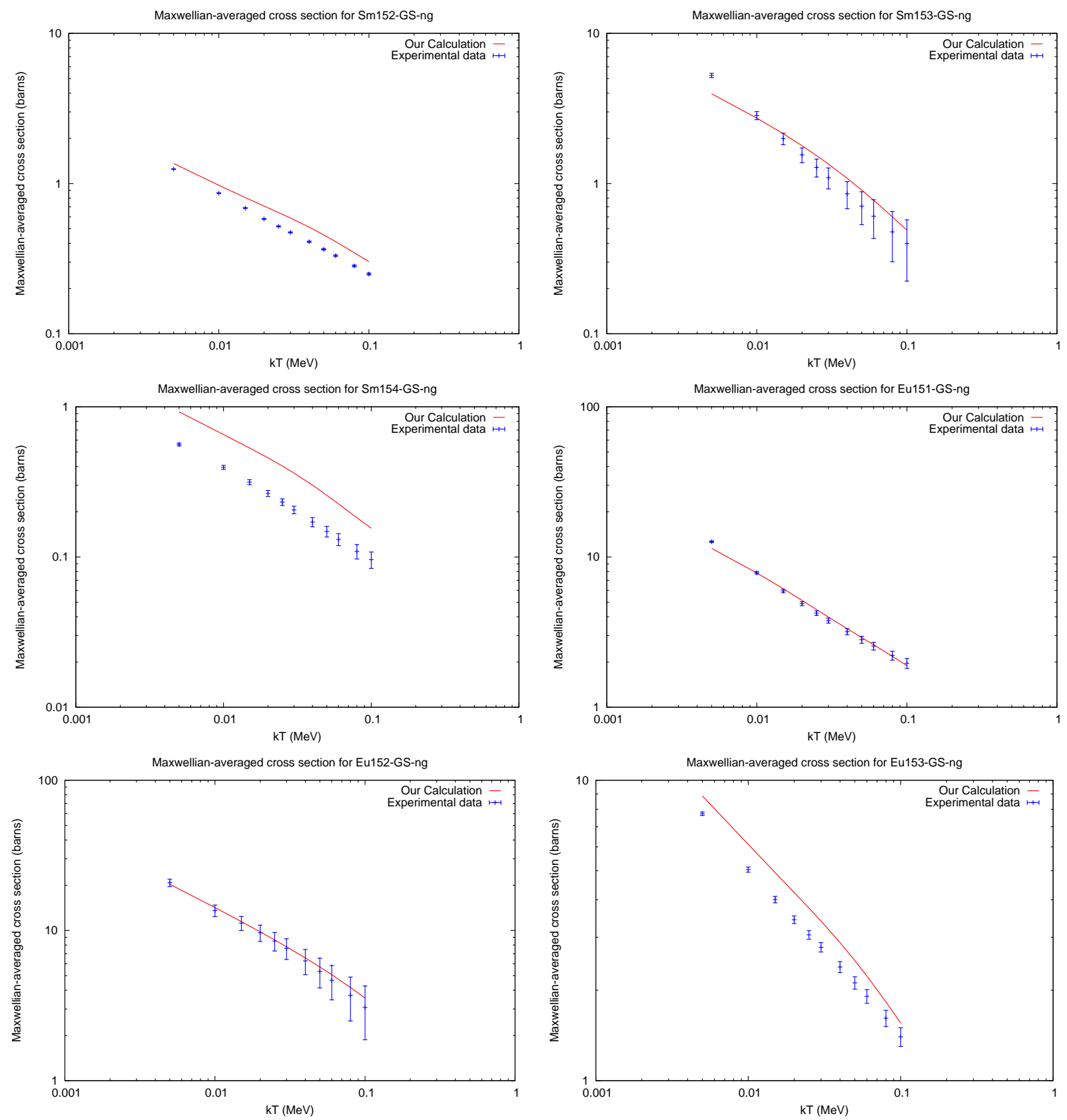

Fig. 22.- (continued) 

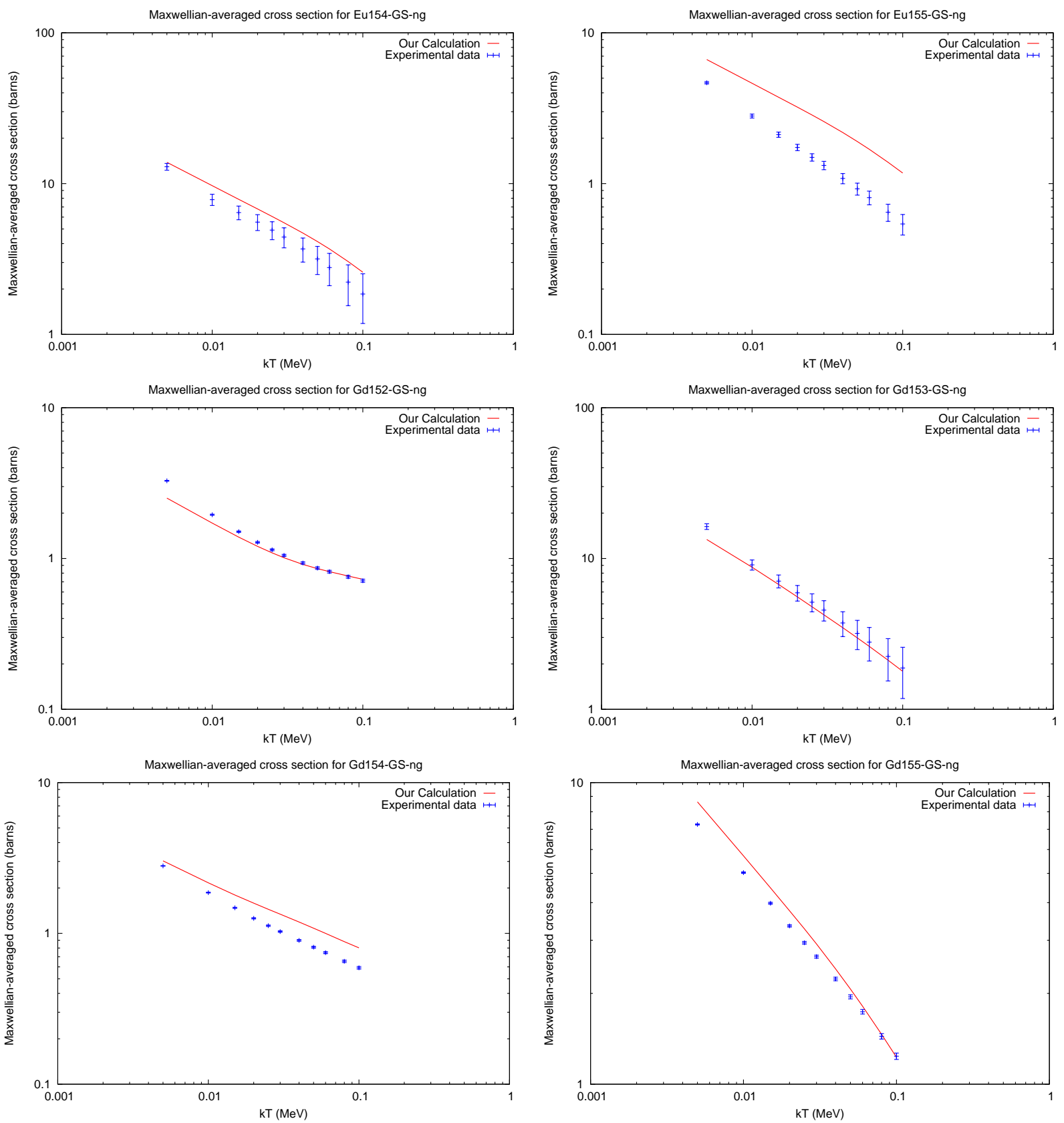

Fig. 22.- (continued) 

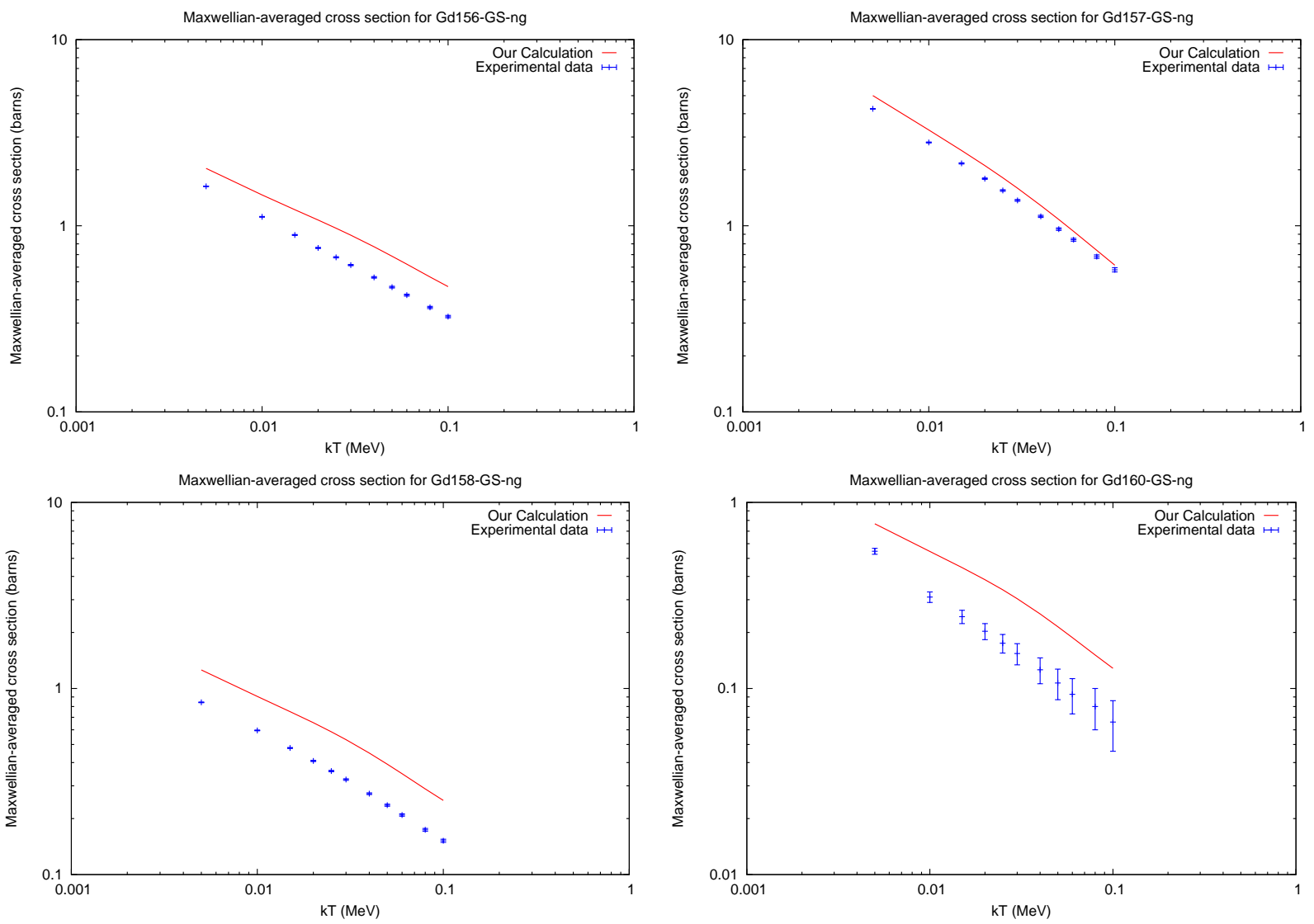

Fig. 22.- (continued) 



Fig. 23.- Measured vs. calculated $(\mathrm{n}, 2 \mathrm{n})$ cross sections for select targets. 

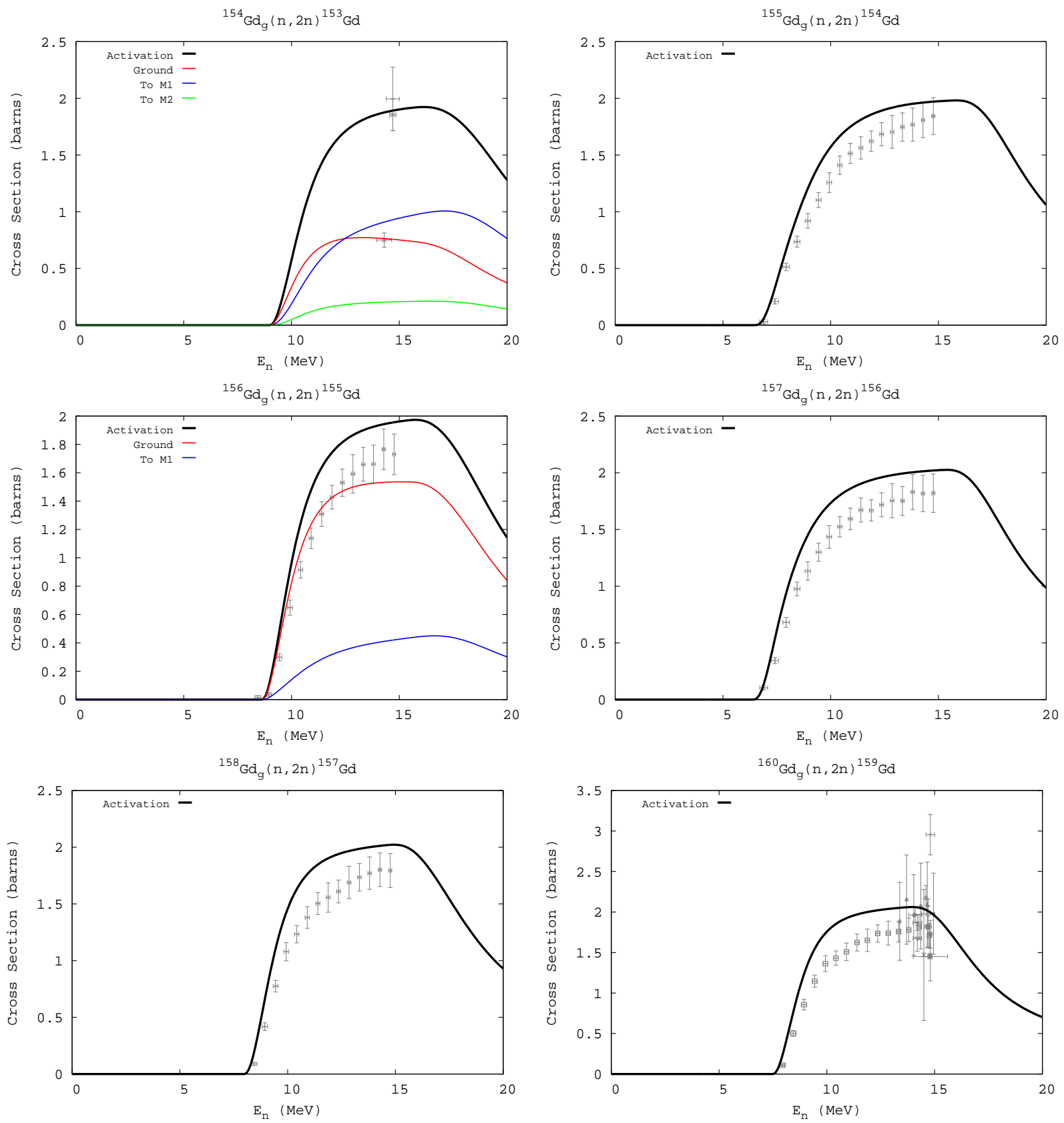

Fig. 23.- (continued) 

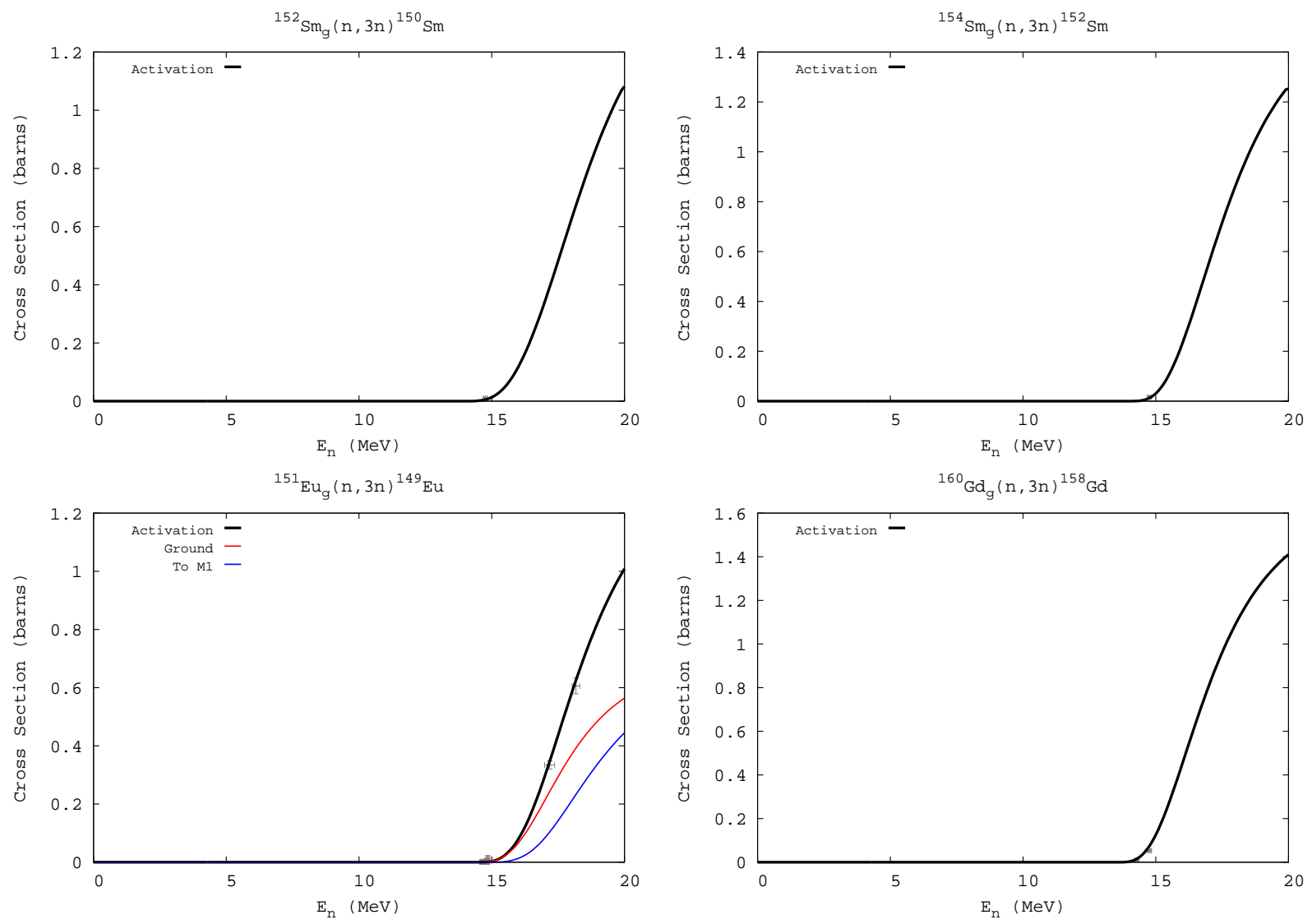

Fig. 24.- Measured vs. calculated (n,3n) cross sections for select targets. 

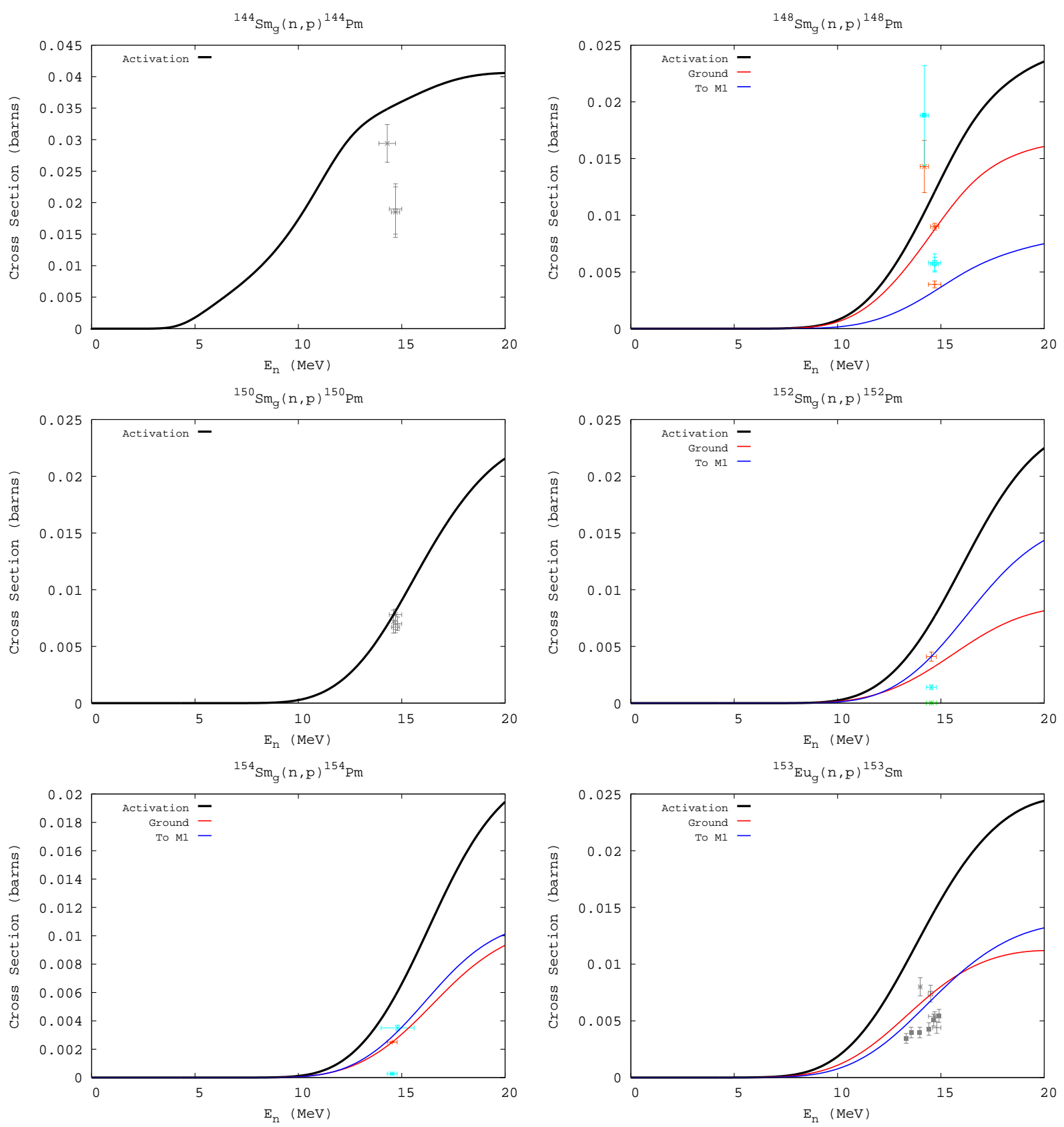

Fig. 25.- Measured vs. calculated (n,p) cross sections for select targets. 

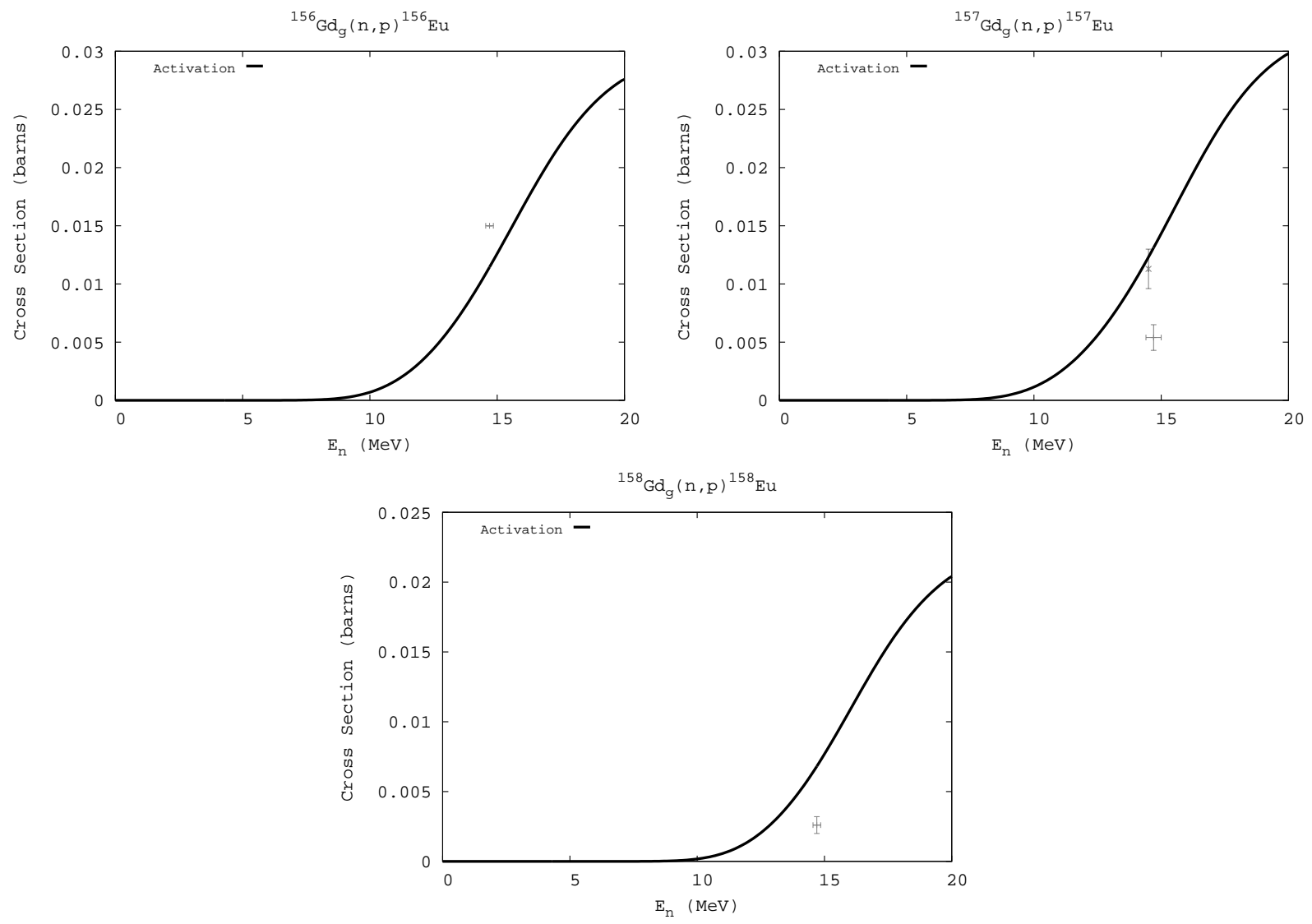

Fig. 25.- (continued) 

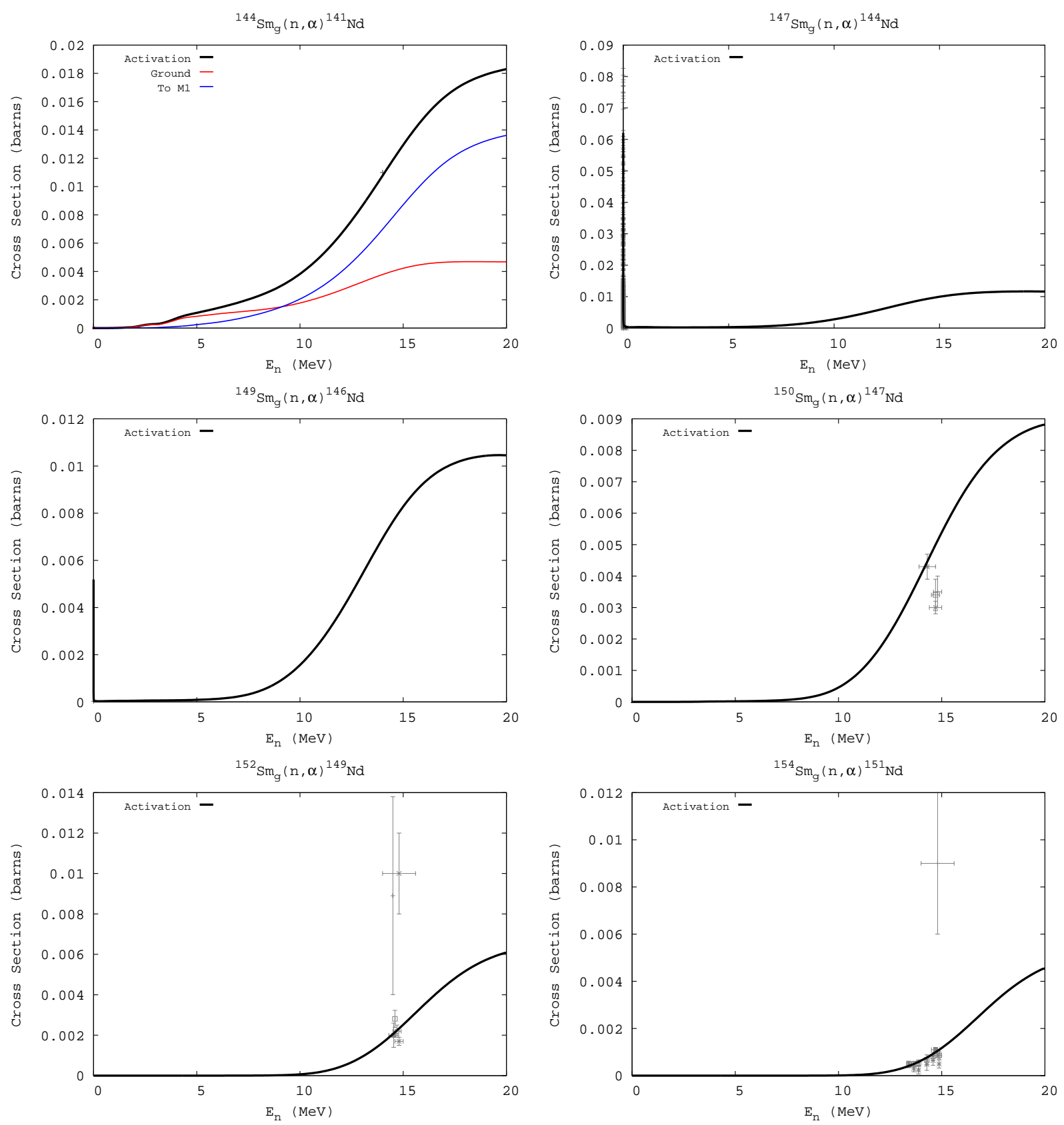

Fig. 26.- Measured vs. calculated $(\mathrm{n}, \alpha)$ and $(\mathrm{n}, \mathrm{d})$ cross sections for select targets. 

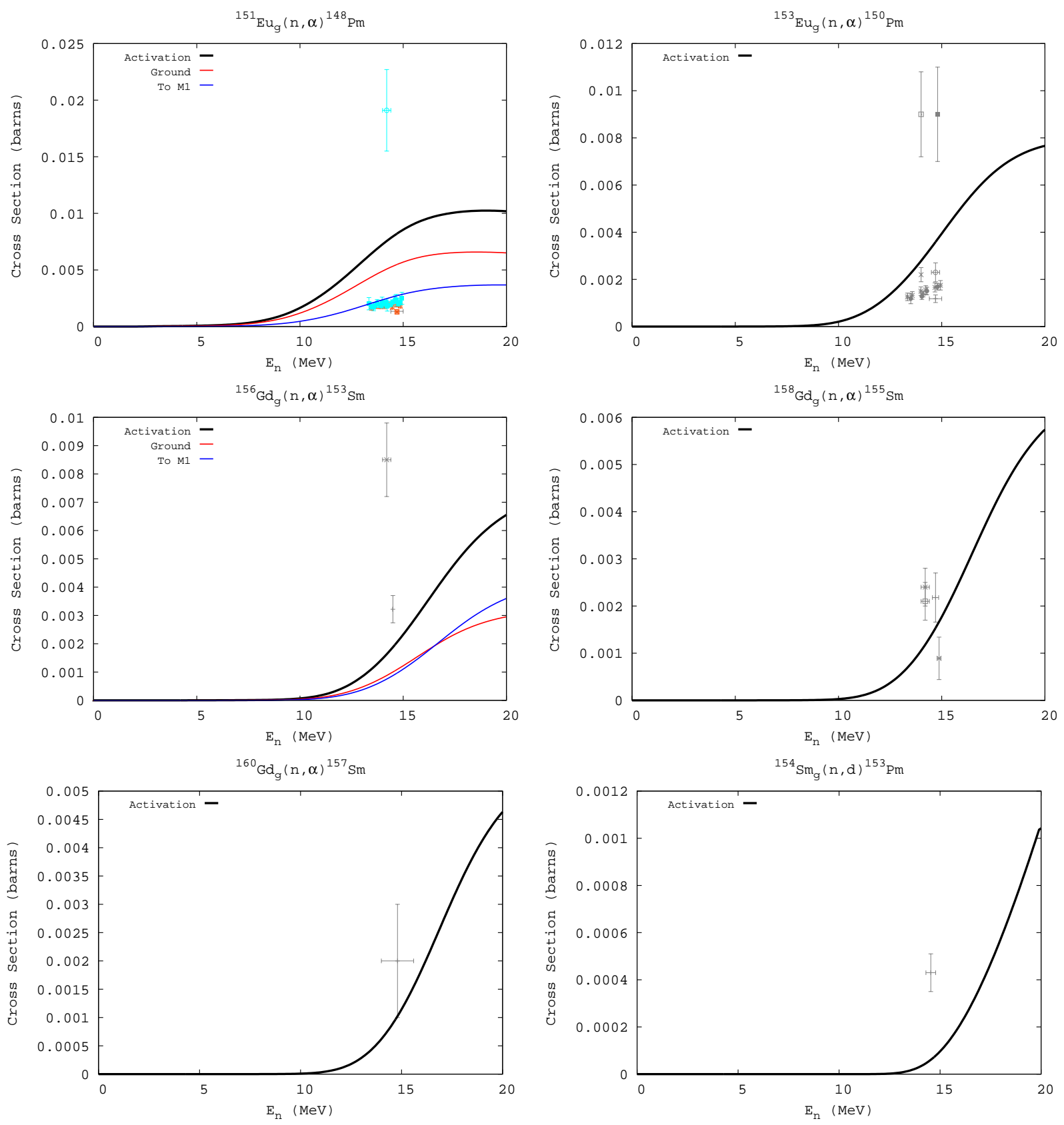

Fig. 26.- (continued) 
C. Modeled Cross Sections: Production and Destruction Channels 

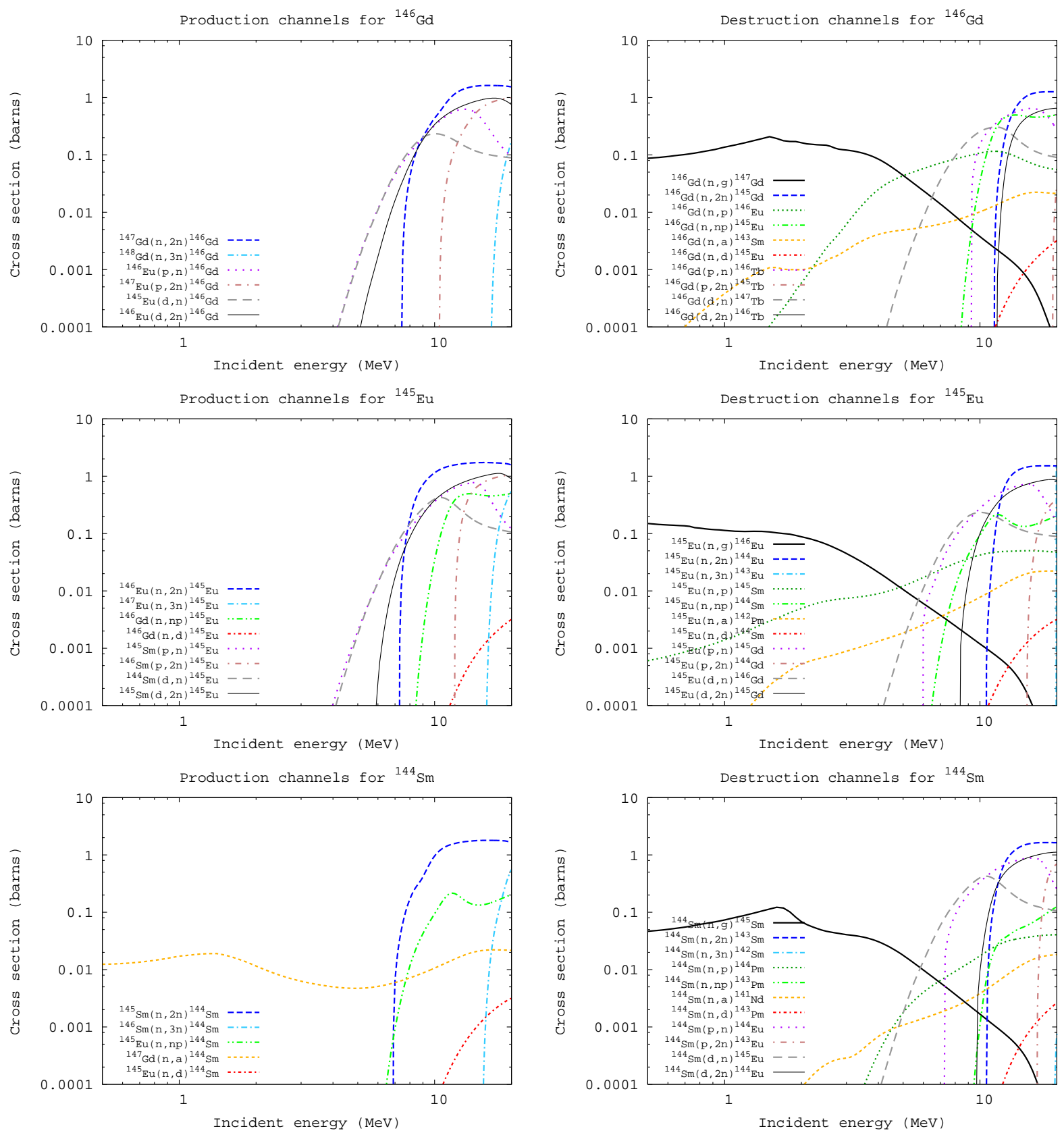

Fig. 27.- Production and destruction cross sections for Sm, Eu, \& Gd targets 

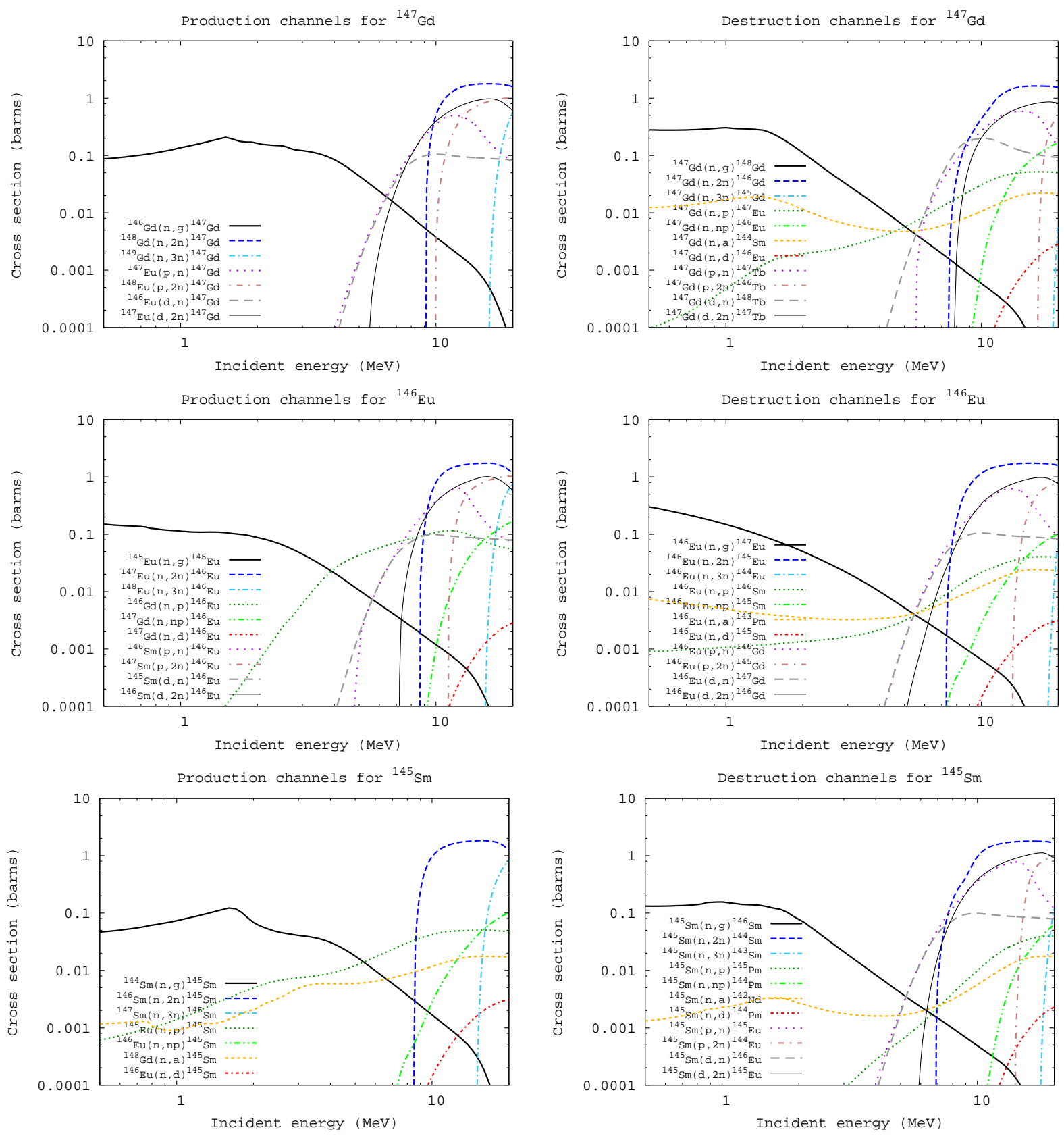

Fig. 27.- (continued) 

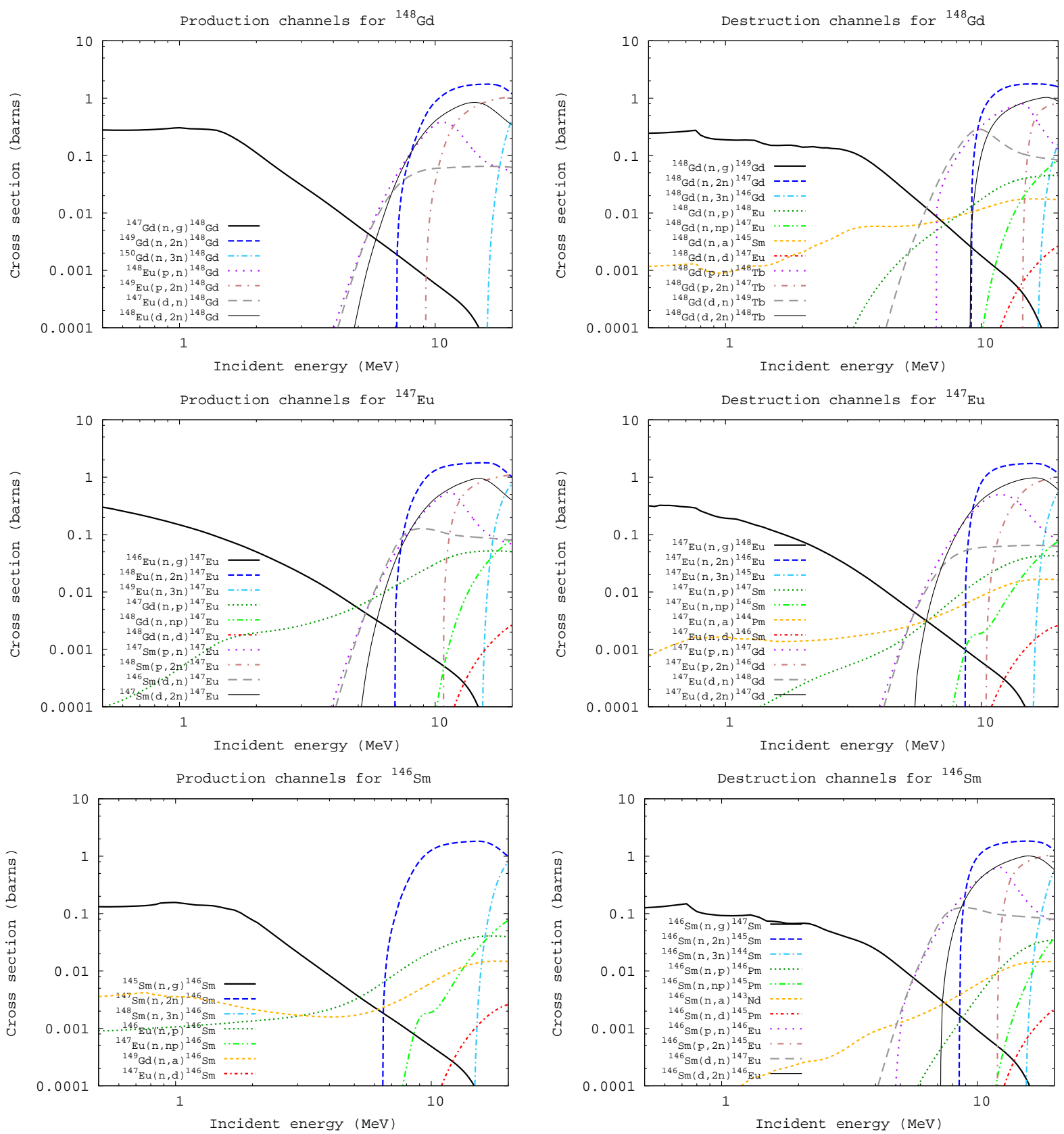

Fig. 27.- (continued) 

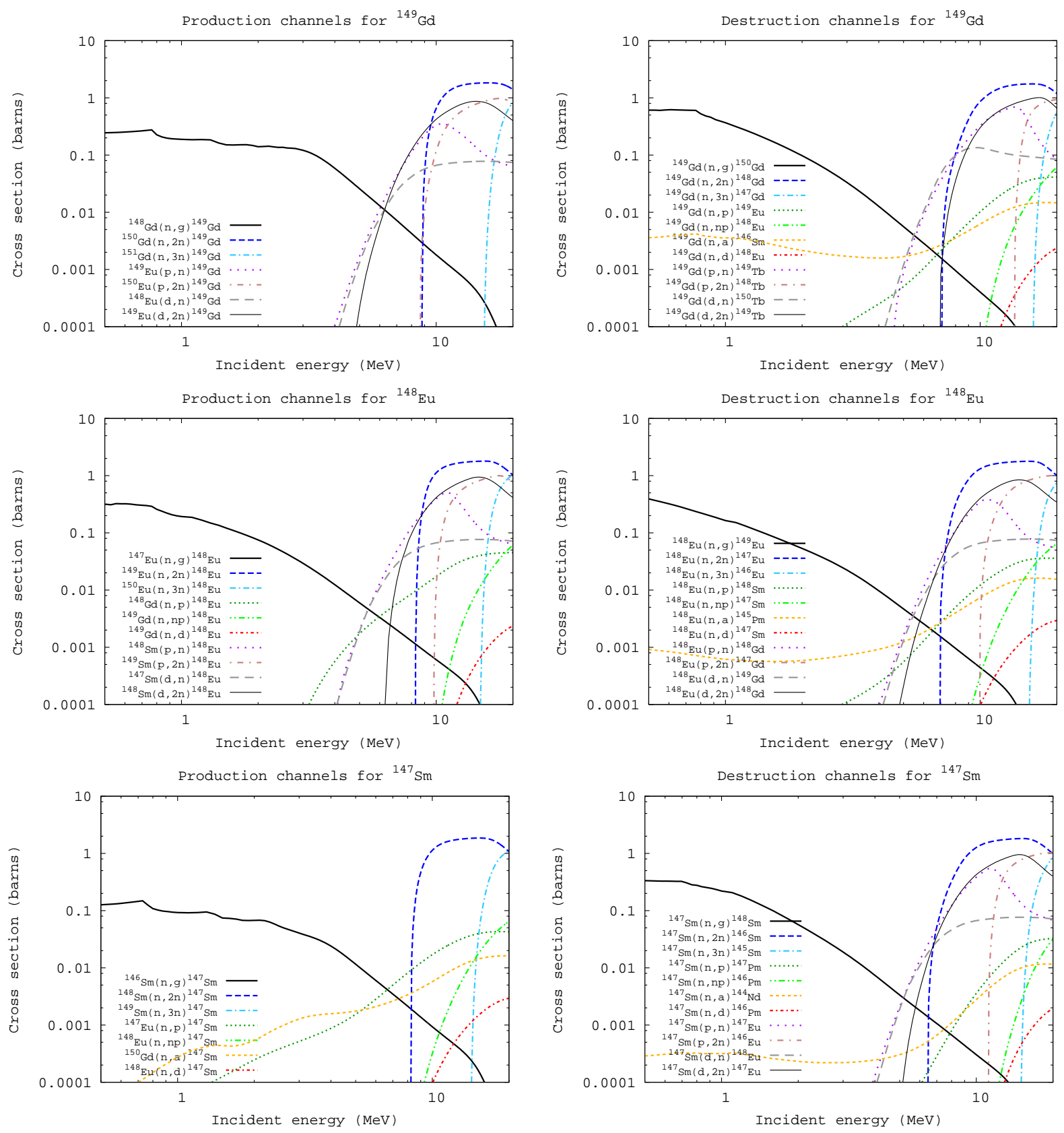

Fig. 27.- (continued) 



Fig. 27.- (continued) 

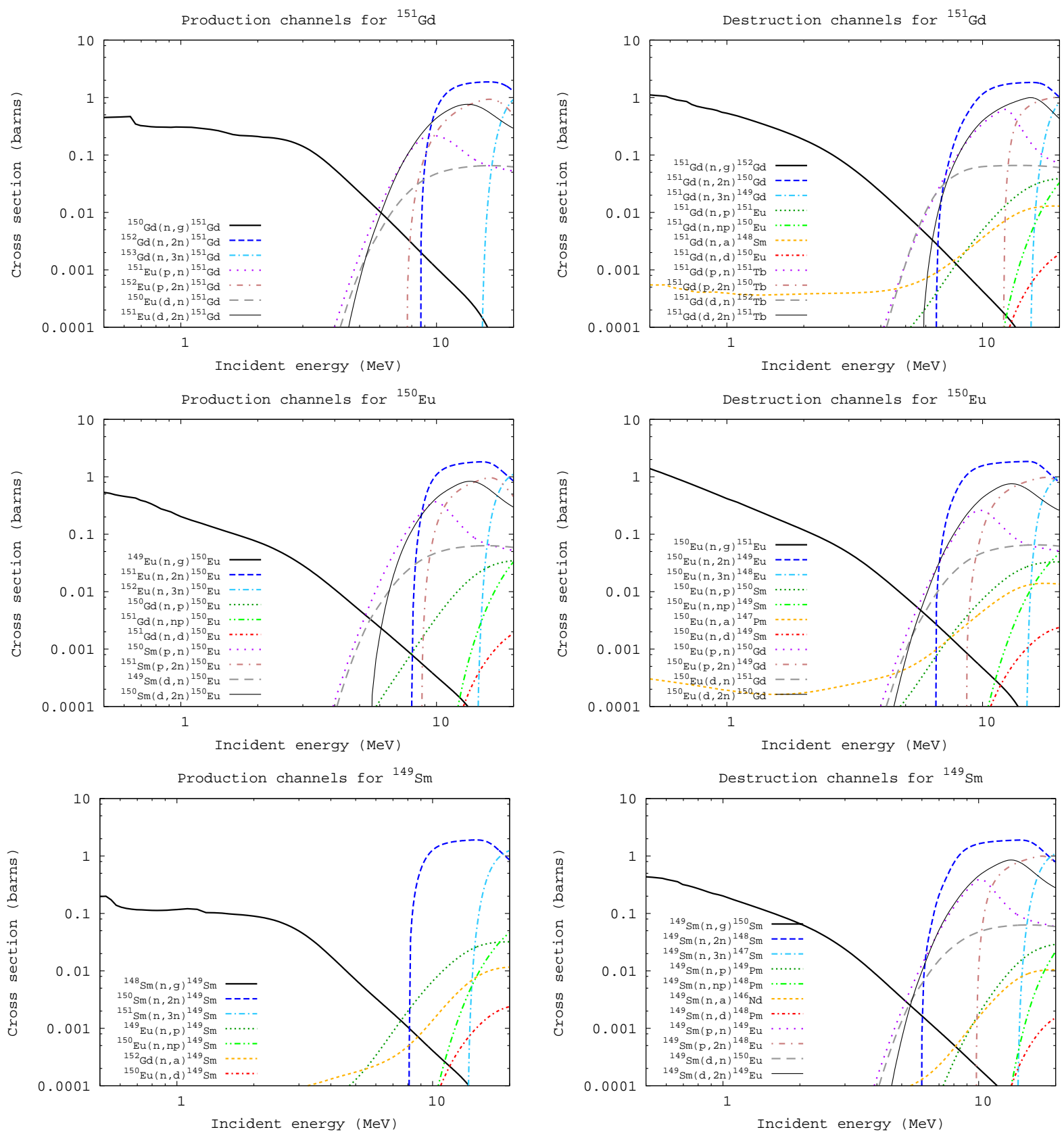

Fig. 27.- (continued) 

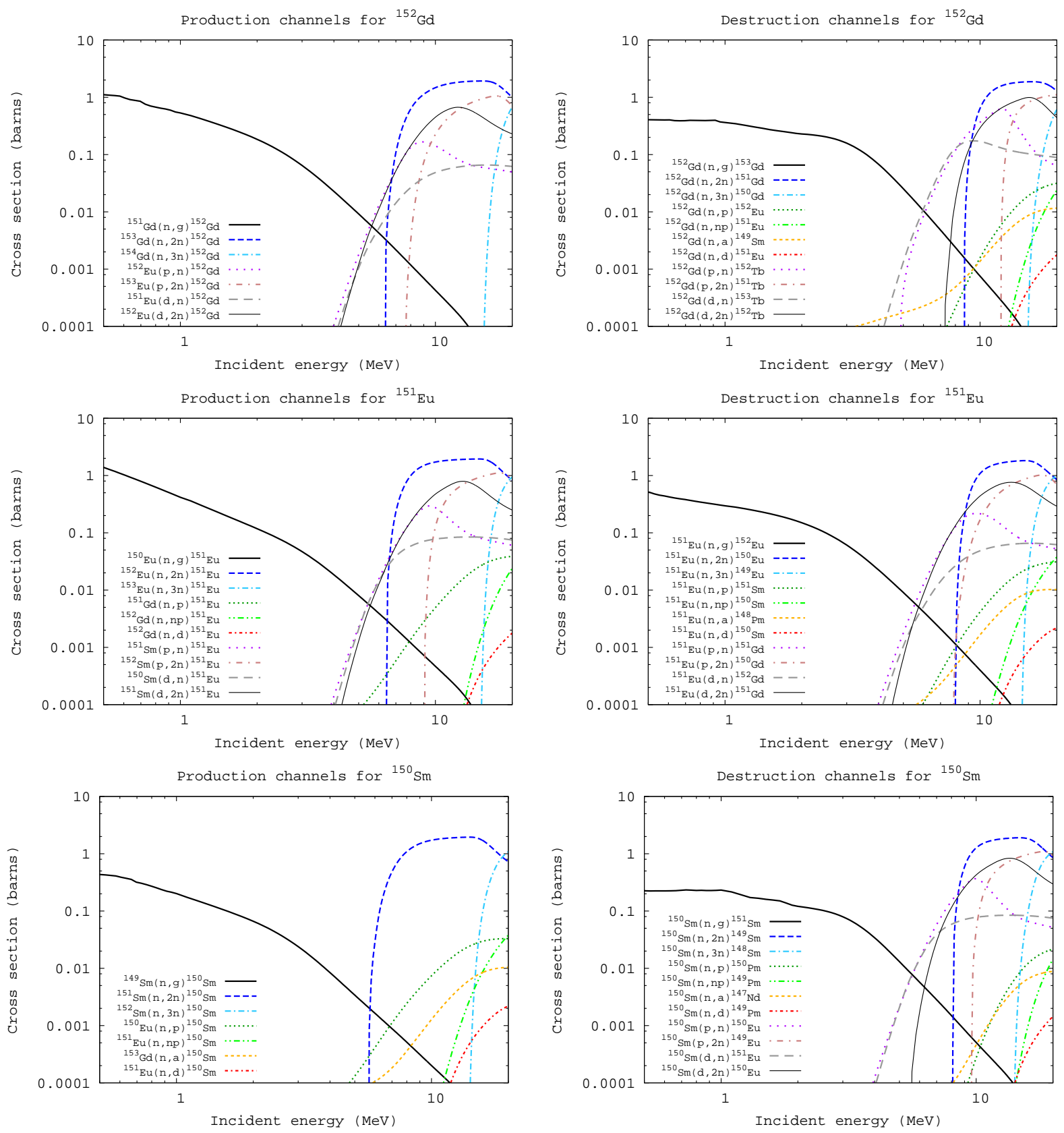

Fig. 27.- (continued) 

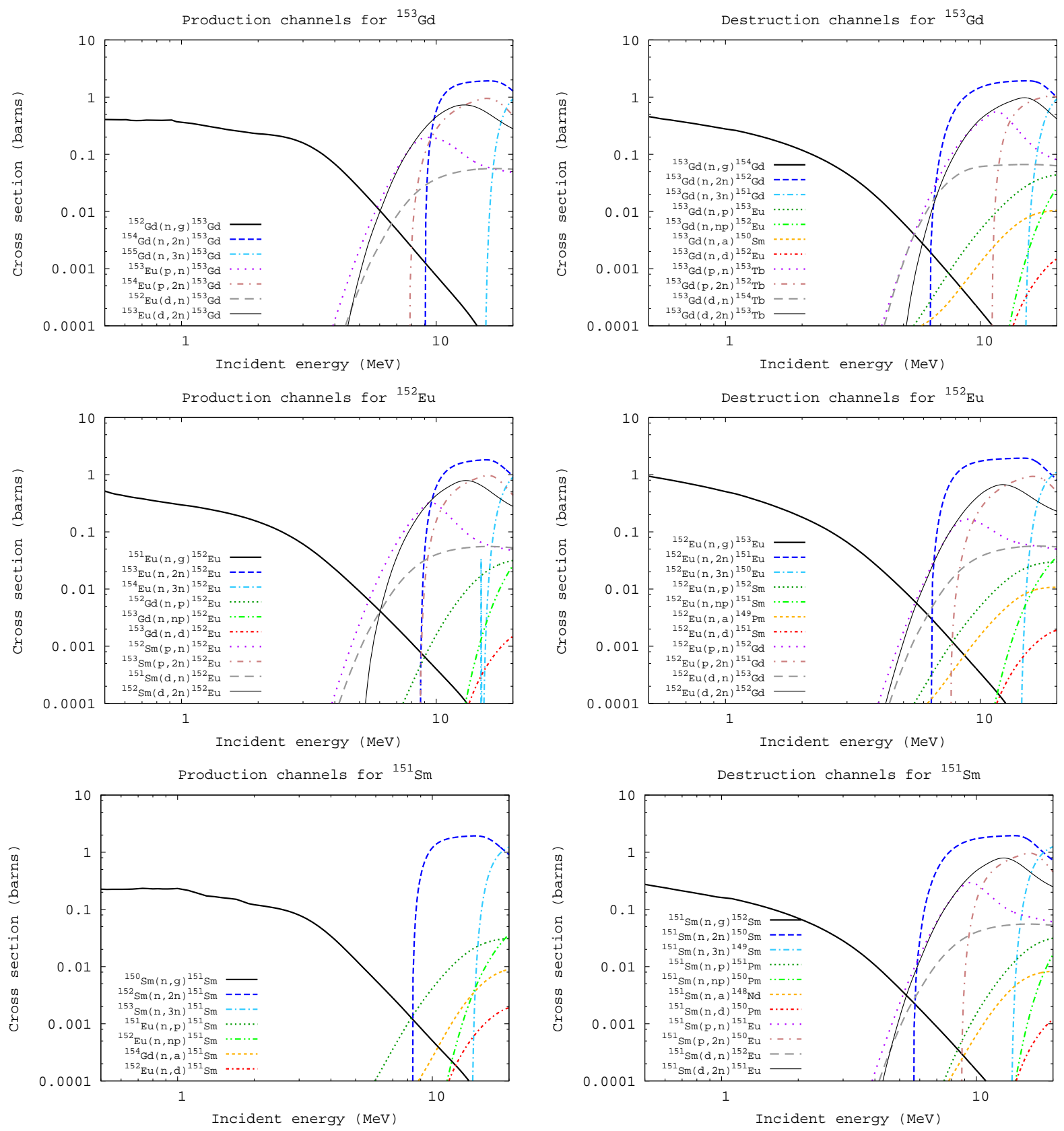

Fig. 27.- (continued) 

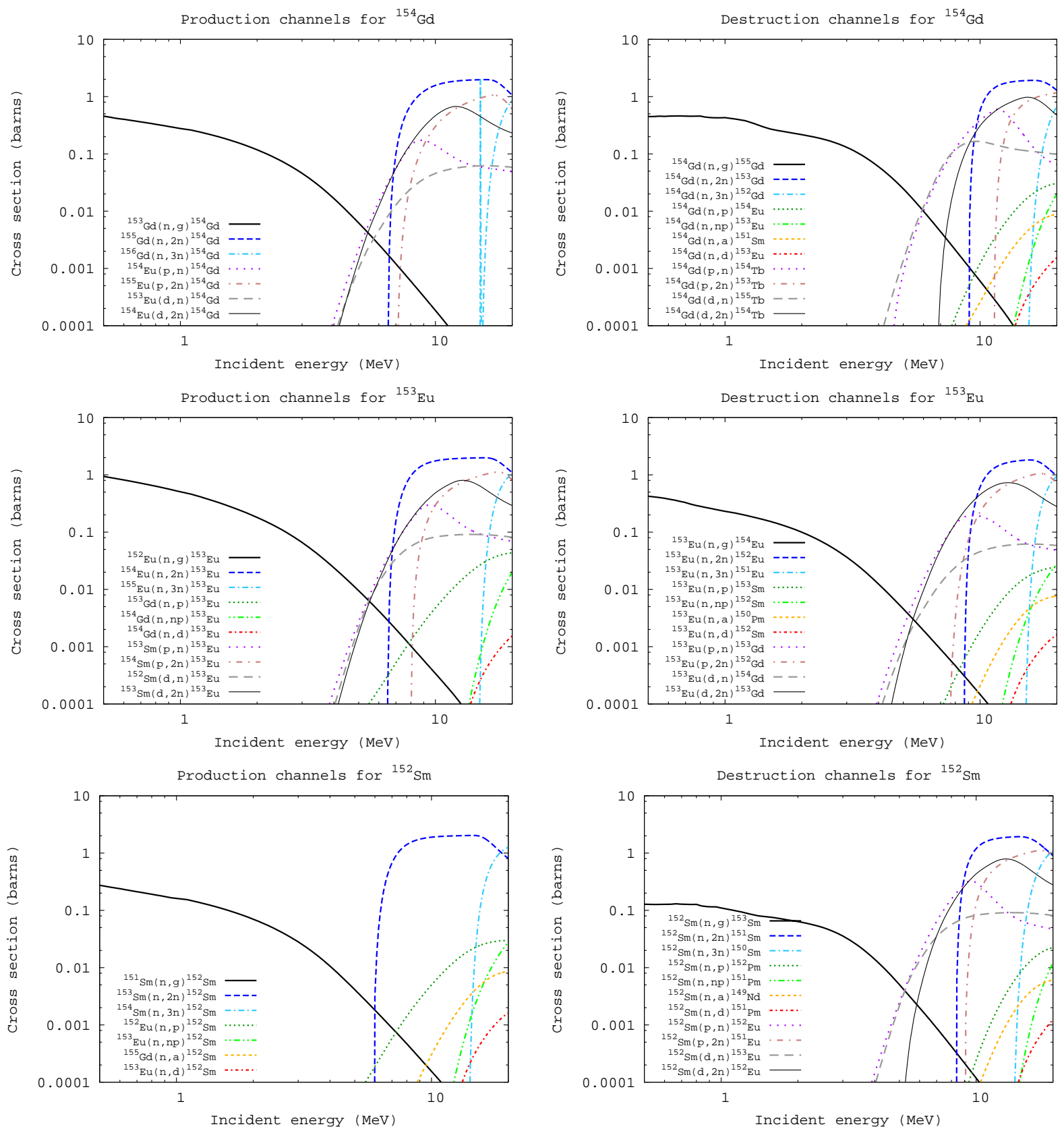

Fig. 27.- (continued) 

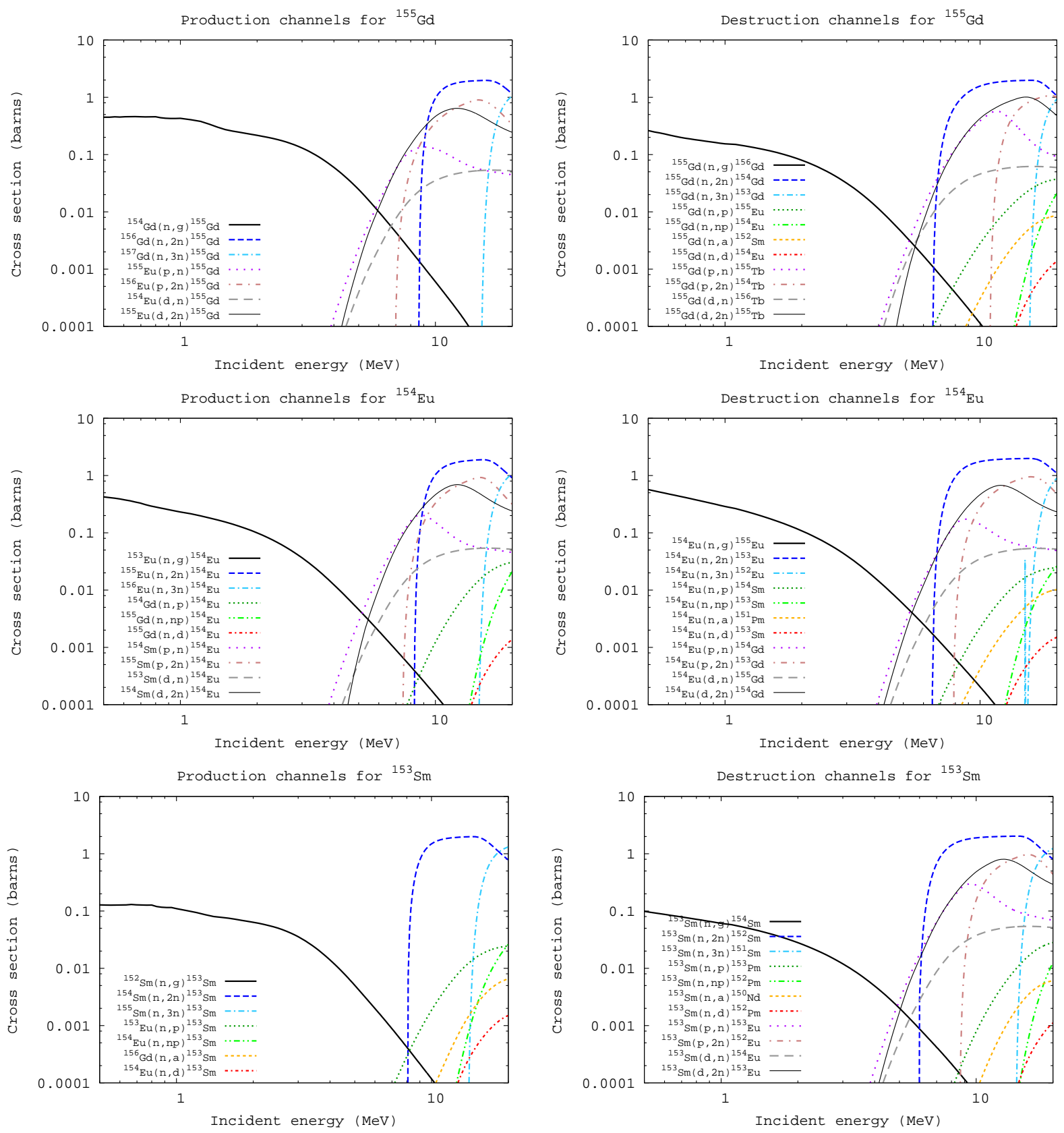

Fig. 27.- (continued) 

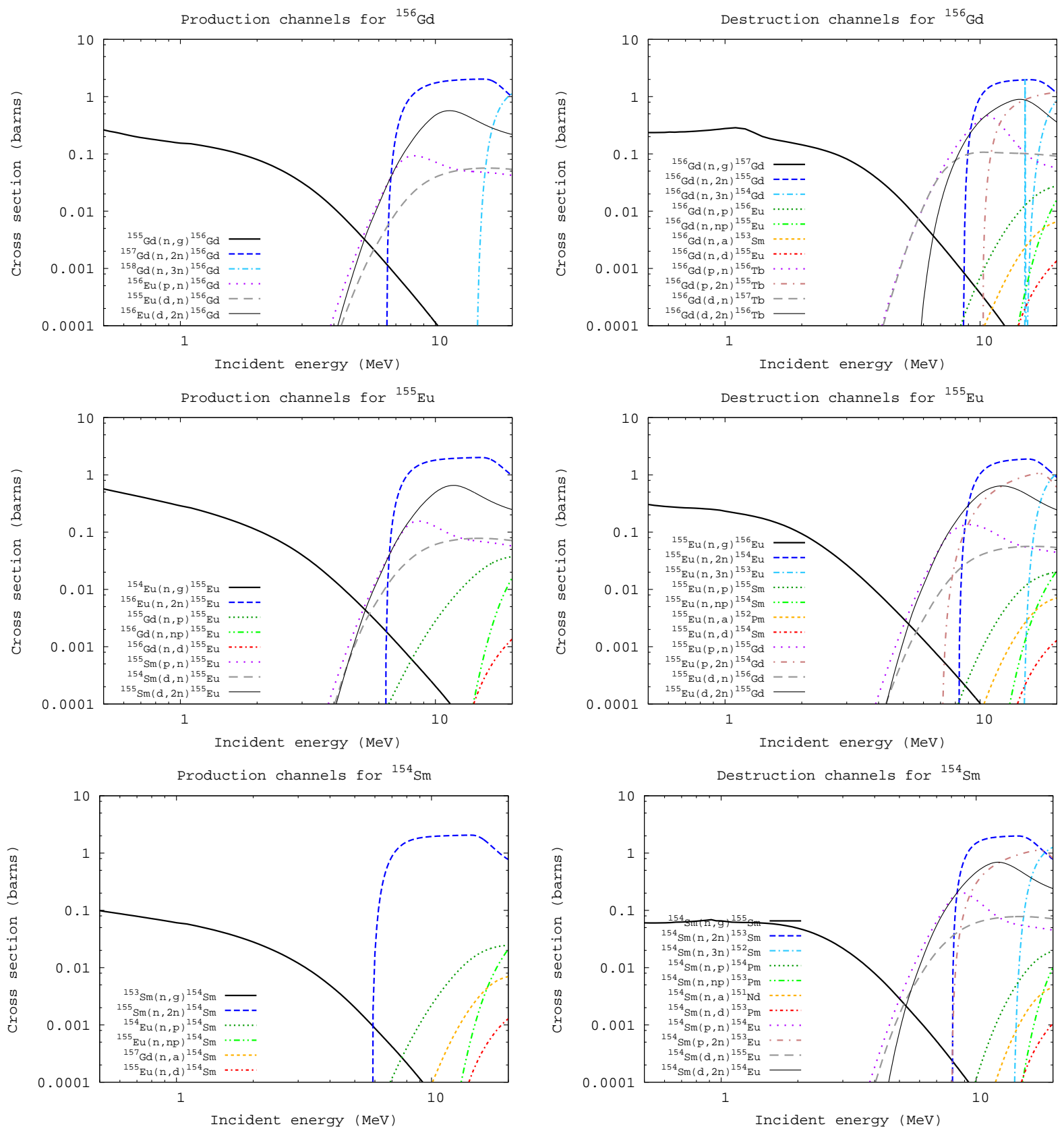

Fig. 27.- (continued) 

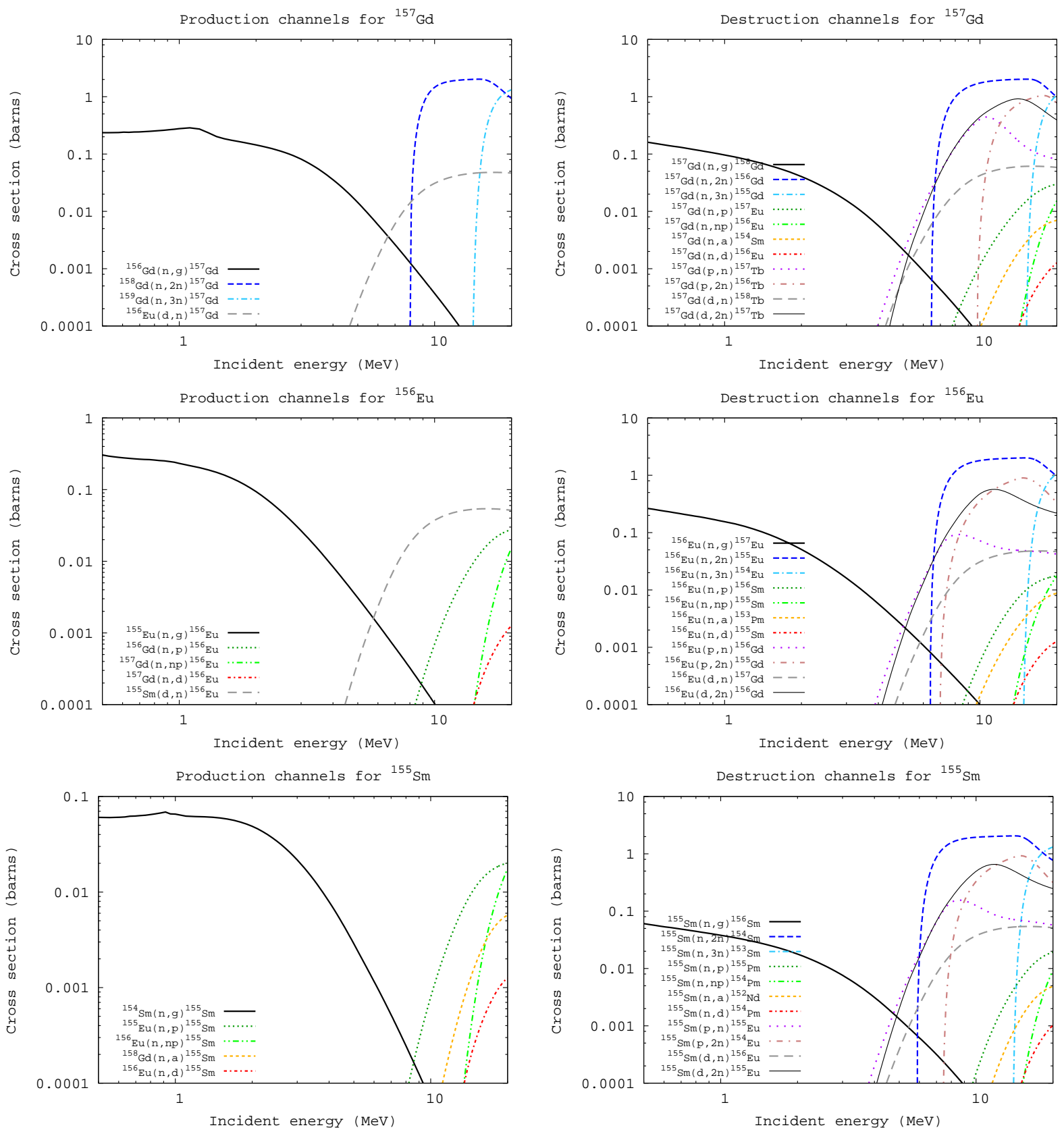

Fig. 27.- (continued) 